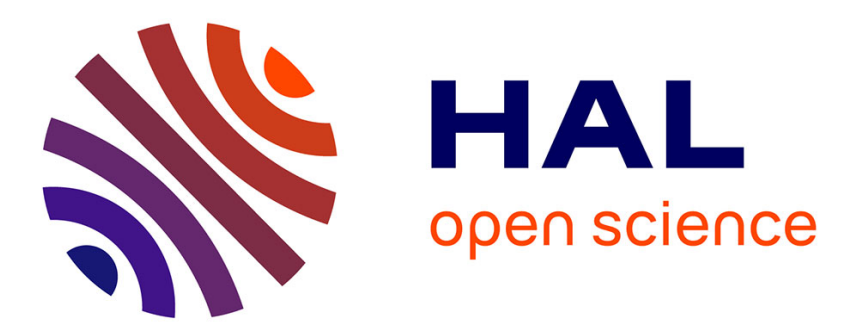

\title{
Near-field internal wave beams in two dimensions
} Bruno Voisin

\section{To cite this version:}

Bruno Voisin. Near-field internal wave beams in two dimensions. Journal of Fluid Mechanics, 2020, 900, pp.A3. 10.1017/jfm.2020.442 . hal-02943847

\section{HAL Id: hal-02943847 https://hal.science/hal-02943847}

Submitted on 20 Sep 2020

HAL is a multi-disciplinary open access archive for the deposit and dissemination of scientific research documents, whether they are published or not. The documents may come from teaching and research institutions in France or abroad, or from public or private research centers.
L'archive ouverte pluridisciplinaire HAL, est destinée au dépôt et à la diffusion de documents scientifiques de niveau recherche, publiés ou non, émanant des établissements d'enseignement et de recherche français ou étrangers, des laboratoires publics ou privés. 


\title{
Near-field internal wave beams in two dimensions
}

\author{
Bruno Voisin ${ }^{1} \dagger$ \\ ${ }^{1}$ Laboratoire des Écoulements Géophysiques et Industriels, \\ Univ. Grenoble Alpes, CNRS, Grenoble INP, 38000 Grenoble, France
}

(Received xx; revised xx; accepted xx)

A new theory is presented for the generation of two-dimensional internal wave beams, including the effects of viscosity and unsteadiness on the propagation of the waves, and extending to the near field the classical theory of Lighthill for the far field. For this, the forcing is assumed to be of compact support. Several equivalent expressions of the waves are obtained, each associated with the choice of a support of simple shape embedding the actual support of the forcing. When the two match, the expression of the waves is valid everywhere in the fluid. For an oscillating body, the existence of critical points where the waves rays are tangential to the body is correctly accounted for, an essential requirement with regard to later inclusion of nonlinear effects and boundary layer eruption into the analysis, both of which take their origin at the critical points. Embedding supports in the shape of a circle, an ellipse and a strip are considered. Line forcing is also considered, on a weaker assumption of rapid decrease at infinity. The analysis reduces to the classical analysis of Hurley \& Keady in the isotropic case of an oscillating circular cylinder, and is otherwise applied to four anisotropic oscillating bodies: an elliptic cylinder, a vertical plate, a vertical wave generator and a thin Gaussian bump.

Key words: To be added during the typesetting process

\section{Introduction}

Internal gravity waves in density-stratified fluids, and the similar inertial waves in rotating fluids, first came to the attention of the scientific community owing to the striking pattern, a St. Andrew's Cross, that they form under oscillatory forcing; a pattern predicted and visualized by Görtler (1943) and Mowbray \& Rarity (1967) for internal waves, and Görtler (1944) and Oser (1958) for inertial waves. For several decades their understanding rested on group velocity ideas, put into quantitative use by Lighthill $(1978, \S 4.10)$ in the far field, namely at large distances from the forcing. The analyses of the internal shear layers that develop at the cross edges by Thomas \& Stevenson (1972), Walton (1975), Rieutord, Georgeot \& Valdetarro (2001), Ogilvie (2005), Machicoane et al. (2015), Le Dizès \& Le Bars (2017) and Beckebanze et al. (2018), together with the analyses of nonlinear effects by Tabaei \& Akylas (2003) and Kataoka \& Akylas (2015), all involve a far-field assumption in one way or another.

The advent of quantitative measurement techniques such as synthetic schlieren for density disturbances (Sutherland et al. 1999; Dalziel, Hughes \& Sutherland 2000) and particle image velocimetry for fluid velocities (Westerweel 1997) showed the need for a

$\dagger$ Email address for correspondence: bruno.voisin@univ-grenoble-alpes.fr 
theory valid not only in the far field, but also in the near field, close to the forcing. When the forcing is a body of simple shape, oscillating in an inviscid fluid, a combination of coordinate stretching and analytic continuation allows the calculation of the waves at arbitrary distance from the body. This method, introduced by Bryan (1889) for inertial waves and Hurley (1972) for internal waves, has been applied to circular and elliptic cylinders by Hurley $(1972,1997)$ and Appleby \& Crighton (1986), and to spheres and spheroids by Hendershott (1969), Krishna \& Sarma (1969), Sarma \& Krishna (1972), Lai \& Lee (1981), Appleby \& Crighton (1987), Voisin (1991), Rieutord et al. (2001) and Davis (2012). The waves manifest themselves as a set of critical rays, with singular amplitude at the rays and phase jumps across them.

Comparison with experiment requires the inclusion of viscosity, to smooth out the singularities. A decisive contribution has been made by Hurley \& Keady (1997), who rewrote Hurley's (1997) inviscid solution for the elliptic cylinder as a spectral integral, and added, at each wavenumber, Lighthill's $(1978, \S 4.10)$ viscous attenuation factor for the far field into it; see also Sutherland $(2010, \S 5.2)$. Quantitative agreement has been found excellent with experiments involving a circular cylinder, both in the far field (Sutherland et al. 1999, 2000) and in the near field (Zhang, King \& Swinney 2007). The agreement was more qualitative for an elliptic cylinder (Sutherland \& Linden 2002), but remained consistent with what can be expected from a linear theory. As a result, the idea has emerged that Lighthill's far-field picture of the effect of viscosity applied to all oscillating bodies, everywhere in the fluid.

A different picture has been obtained, however, using direct calculation for thin forcing, namely line forcing in two dimensions and plane forcing in three dimensions. Lighthill's $(1978, \S 4.10)$ theory predicts that the evolution of the waves away from the forcing is set by the distance along the axes of the St. Andrew's Cross: in the inviscid case, the determination of the multivalued functions involved in the expression of the waves depends on this distance; in the viscous case, the attenuation of the waves at each wavenumber depends on it. By contrast, for thin forcing, the evolution of the waves is set by the distance normal to the forcing. This can be seen in the inviscid calculations of Oser (1957), Reynolds (1962), Martin \& Llewellyn Smith (2011, 2012b) and Davis (2012) for a horizontal disc, Hurley (1969) for an inclined plate and Llewellyn Smith \& Young (2003) for a vertical plate, or in the viscous calculations of Kistovich \& Chashechkin $(1999 a, b)$ for a two-dimensional inclined plate, Vasil'ev \& Chashechkin (2003, 2006a, $b$, 2012) for a three-dimensional inclined plate, Tilgner (2000), Bardakov, Vasil'ev \& Chashechkin (2007), Davis \& Llewellyn Smith (2010), Le Dizès (2015) and Le Dizès \& Le Bars (2017) for a horizontal disc, Maurer et al. (2017) and Boury, Peacock \& Odier (2019) for a horizontal wave generator and Beckebanze, Raja \& Maas (2019) for a vertical wave generator. To some extent this can also be seen in the inviscid calculations of Gabov (1985) for a horizontal plate, Gabov \& Pletner (1985) for an inclined plate, Gabov \& Krutitskii (1987) for a vertical plate and Gabov \& Pletner (1988) for a horizontal disc, although only Gabov \& Pletner (1985) considered the determination of the multivalued functions explicitly.

Different measures have been taken to reconcile the two pictures with each other. For the waves generated by oscillatory flow over an isolated Gaussian bump at the ocean bottom, Peacock, Echeverri \& Balmforth (2008) used the analysis of Balmforth, Ierley \& Young (2002) for periodic bottom topography, obtaining first a viscous attenuation factor depending on the vertical coordinate, then switching to one depending on the along-cross coordinate, attributing the switch to the change from periodic to isolated topography. Kistovich \& Chashechkin $(1994,1995)$ considered the reflection of the wave beam generated by a point source at an inclined plane; they obtained first a reflected 
beam whose integral expression included a viscous attenuation factor depending on the normal coordinate to the plane, then changed variable and deformed the contour of integration in the complex wavenumber plane to switch to a new expression in which the viscous attenuation factor depended on the along-beam coordinate.

The present paper is part of a two-step effort to build a new theory of the generation of internal waves by an oscillating body, valid for low viscosity and at arbitrary distance from the body. First, applying the boundary integral method, a representation of the body is devised as a distribution of singularities at its surface; at this stage, viscosity is ignored. The theoretical foundations of the method have been discussed by Kapitonov (1980), Skazka (1981), Gabov \& Shevtsov $(1983,1984)$ and Martin \& Llewellyn Smith (2012a). The method has been applied analytically to a horizontal plate by Gabov (1985), an inclined plate by Gabov \& Pletner (1985), one or several vertical plates by Gabov \& Krutitskii (1987), Llewellyn Smith \& Young (2003), Nycander (2006) and Musgrave et al. (2016), a horizontal disc by Gabov \& Pletner (1988) and a circular cylinder by Sturova (2001). Numerically it has been applied to various topographies by Pétrélis, Llewellyn Smith \& Young (2006), Balmforth \& Peacock (2009), Echeverri \& Peacock (2010) and Echeverri et al. (2011), and to circular and elliptic cylinders by Sturova (2006, 2011). Secondly, the representation being known, Fourier analysis is used to calculate the waves that it generates in a viscous fluid. This procedure may be viewed as a generalization and systematization of the approach of Hurley (1997) and Hurley \& Keady (1997).

We consider the second step here, in the two-dimensional case. Section 2 presents the classical approach of Lighthill $(1978, \S 4.10)$ for the far field and discusses its extension to the near field; the forcing is assumed isotropic, namely of circular shape. After a brief derivation of the wave equation in $\S 3$, the simplest type of anisotropy is investigated in $\S 4$, namely forcing of elliptic shape. As will be seen, it is not meant by this that the source function needs to be exactly in the shape of a circle in $\S 2$ and an ellipse in $\S 4$, but, rather, that its support is included inside this shape for the duration of the calculation. The particular case of line forcing is considered in $\S 5$. The use of alternative integration strategies, yielding simpler expressions valid in less extended domains, is presented in $\S 6$, while $\$ 7$ discusses unsteady effects. Section 8 applies the theory to four anisotropic sources of particular interest, for which experimental measurements are available: an elliptic cylinder, a vertical barrier, a wave generator and a thin Gaussian bump. Finally $\$ 9$ discusses the relevance of the approach, and points out the usefulness of the Green's function method. It is followed by Appendix A presenting the modifications to the theory when, as is generally the case, the source function is not a standard function but a distribution, and by Appendix B calculating the Green's function.

\section{Wave structure}

\subsection{Inviscid case}

Any quantity, $\psi$ say, associated with internal gravity waves in an inviscid uniformly stratified Boussinesq fluid satisfies an equation of the form

$$
\left(\frac{\partial^{2}}{\partial t^{2}} \nabla^{2}+N^{2} \nabla_{\mathrm{h}}^{2}\right) \psi=q,
$$

where $N$ is the buoyancy frequency, $z$ the vertical coordinate, $\boldsymbol{\nabla}=(\partial / \partial x, \partial / \partial y, \partial / \partial z)$ the del operator, $\nabla_{\mathrm{h}}=(\partial / \partial x, \partial / \partial y, 0)$ its original projection and $q$ a source term; see for example Lighthill $(1978, \S 4.1)$ or Voisin (1991). Assuming the source to be twodimensional and monochromatic, $q=f(x, z) \exp \left(-\mathrm{i} \omega_{0} t\right)$ with $\omega_{0}<N$, and introducing 
Fourier transforms according to

$$
\begin{gathered}
f(k, m)=\iint f(x, z) \exp [-\mathrm{i}(k x+m z)] \mathrm{d} x \mathrm{~d} z, \\
f(x, z)=\frac{1}{(2 \pi)^{2}} \iint f(k, m) \exp [\mathrm{i}(k x+m z)] \mathrm{d} k \mathrm{~d} m,
\end{gathered}
$$

the solution of (2.1) follows as

$$
\psi=\frac{\exp \left(-\mathrm{i} \omega_{0} t\right)}{4 \pi^{2}} \iint \frac{f(k, m) \exp [\mathrm{i}(k x+m z)]}{\omega_{0}^{2} \kappa^{2}-N^{2} k^{2}} \mathrm{~d} k \mathrm{~d} m,
$$

with $\boldsymbol{x}=(x, z)$ the position, $\boldsymbol{k}=(k, m)$ the wavenumber vector and $r=\left(x^{2}+z^{2}\right)^{1 / 2}$ and $\kappa=\left(k^{2}+m^{2}\right)^{1 / 2}$ their moduli.

Lighthill devised a method for the asymptotic evaluation of such Fourier integral in the far field, as $r \rightarrow \infty$, first for a rapidly decreasing source (1960) then for a source of compact support $(1978, \S 4.9)$, with identical results. We adopt the former, more general presentation; namely, the source function $f(x, z)$ is assumed to decrease asymptotically faster than any inverse power of $x$ or $z$, so that its spectrum $f(k, m)$ is a regular function of the real variables $k$ and $m$.

The asymptotic behaviour of the integral is expressed in terms of the singularities of the integrand (Lighthill 1958, ch. 4). Given the regularity of $f(k, m)$, these are the solutions of the dispersion relation

$$
B\left(\omega_{0}, \boldsymbol{k}\right)=\omega_{0}^{2} \kappa^{2}-N^{2} k^{2}=0 .
$$

In the wavenumber plane this defines a wavenumber curve, represented in figure 1 , in the shape of a St. Andrew's Cross with arms inclined at the angle $\theta_{0}=\arccos \left(\omega_{0} / N\right)$ to the horizontal. Writing

$$
\omega_{0}=N \frac{|k|}{\kappa}, \quad \boldsymbol{c}_{\mathrm{g}}=\left(\frac{\partial \omega_{0}}{\partial k}, \frac{\partial \omega_{0}}{\partial m}\right)=N \frac{m}{\kappa^{3}}(m,-k) \operatorname{sign} k,
$$

the group velocity $\boldsymbol{c}_{\mathrm{g}}$, at which the wave energy propagates, is seen to be perpendicular to $\boldsymbol{k}$. Accordingly, each arm of the cross radiates waves perpendicular to itself, forming another St. Andrew's Cross in the physical plane, represented in figure 2, with arms inclined at the angle $\theta_{0}$ to the vertical.

We introduce characteristic coordinates $\left(x_{ \pm}, z_{ \pm}\right)$such that

$$
x_{ \pm}=x \cos \theta_{0} \mp z \sin \theta_{0}, \quad z_{ \pm}= \pm x \sin \theta_{0}+z \cos \theta_{0},
$$

and associated wavenumbers $\left(k_{ \pm}, m_{ \pm}\right)$such that

$$
k_{ \pm}=k \cos \theta_{0} \mp m \sin \theta_{0}, \quad m_{ \pm}= \pm k \sin \theta_{0}+m \cos \theta_{0},
$$

as shown in figures 1 and 2 . The dispersion relation simplifies to

$$
B\left(\omega_{0}, \boldsymbol{k}\right)=N^{2} m_{+} m_{-}=0,
$$

and the equation of the wavenumber curve to

$$
m_{ \pm}=0
$$

The group velocity becomes

$$
\boldsymbol{c}_{\mathrm{g}}= \pm \frac{N \sin \theta_{0}}{k_{ \pm}} \boldsymbol{e}_{z_{ \pm}}
$$



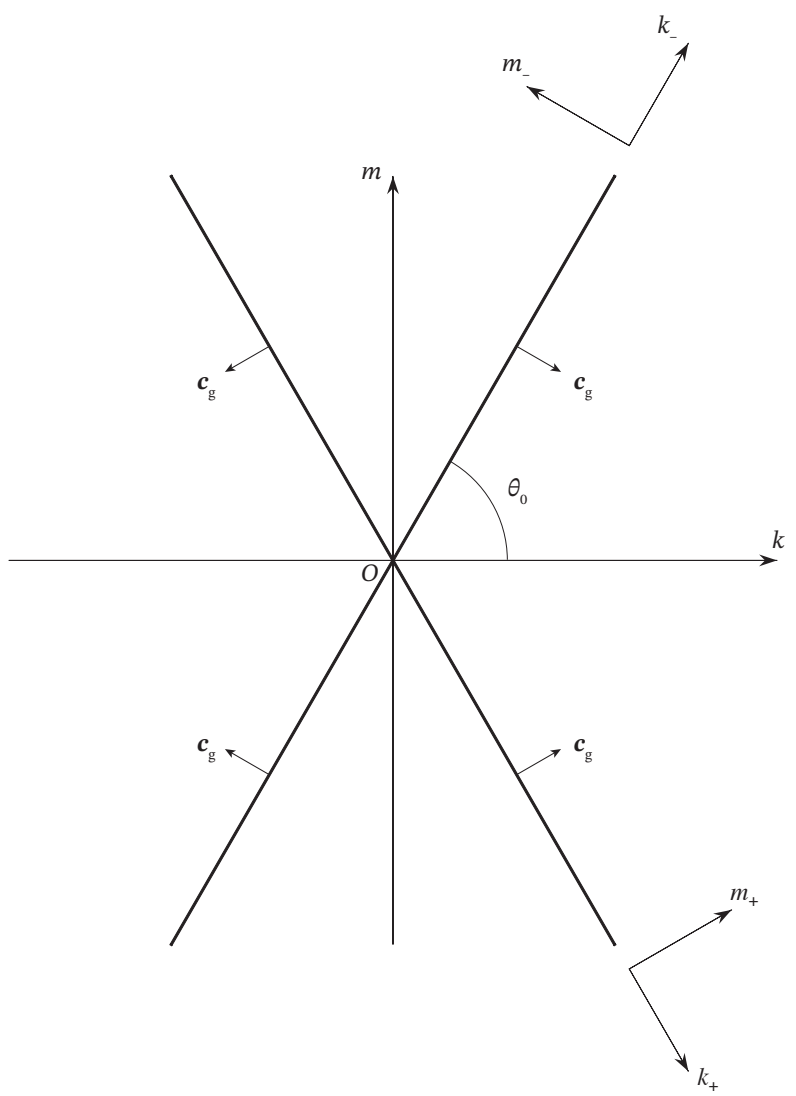

FIGURE 1. Wavenumber curve for two-dimensional internal waves.

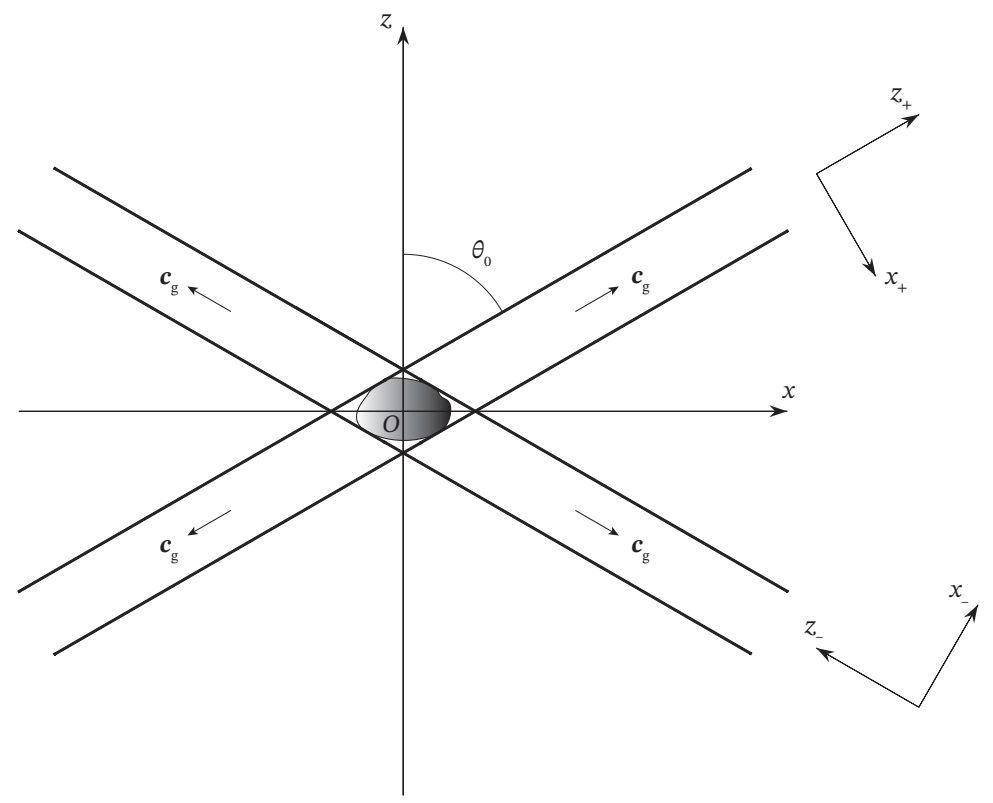

FIGURE 2. Wave beams for two-dimensional internal waves. 
with

$$
\boldsymbol{e}_{z_{ \pm}}= \pm \boldsymbol{e}_{x} \sin \theta_{0}+\boldsymbol{e}_{z} \cos \theta_{0}
$$

a unit vector along the $z_{ \pm}$-axis. The two halves $k_{ \pm}<0$ and $k_{ \pm}>0$ of each arm $m_{ \pm}=0$ of the cross are seen to radiate waves in opposite directions, shown in figure 1.

The contribution of each arm to integral (2.3) is evaluated in coordinates $\left(k_{ \pm}, m_{ \pm}\right)$, writing

$$
\psi=\frac{\exp \left(-\mathrm{i} \omega_{0} t\right)}{4 \pi^{2}} \int_{-\infty}^{\infty} \mathrm{d} k_{ \pm} \exp \left(\mathrm{i} k_{ \pm} x_{ \pm}\right) \int_{-\infty}^{\infty} \frac{f_{ \pm}\left(k_{ \pm}, m_{ \pm}\right)}{B\left(\omega_{0}, \boldsymbol{k}\right)} \exp \left(\mathrm{i} m_{ \pm} z_{ \pm}\right) \mathrm{d} m_{ \pm},
$$

where $f_{ \pm}\left(k_{ \pm}, m_{ \pm}\right)=f(k, m)$, then allowing $m_{ \pm}$to become complex and applying the residue theorem to the inner integral. An additional condition is required to displace the real pole (2.9) slightly off the path of integration. For this, Lighthill $(1960,1978, \S 4.9)$ introduced an innovative formulation of the radiation condition, giving the frequency an infinitesimal positive imaginary part $\epsilon \ll N$. The dispersion relation becomes

$$
B\left(\omega_{0}+\mathrm{i} \epsilon, \boldsymbol{k}\right) \sim N^{2}\left[m_{ \pm} m_{\mp}+2 \mathrm{i} \frac{\epsilon}{N}\left(k_{ \pm}^{2}+m_{ \pm}^{2}\right) \cos \theta_{0}\right]=0,
$$

where

$$
m_{\mp}=\mp k_{ \pm} \sin \left(2 \theta_{0}\right)+m_{ \pm} \cos \left(2 \theta_{0}\right)
$$

providing a second-order equation for $m_{ \pm}$as a function of $k_{ \pm}$. To leading order in $\epsilon / N$, the pole (2.9) is displaced to

$$
m_{ \pm} \sim \pm \mathrm{i} \frac{\epsilon}{N \sin \theta_{0}} k_{ \pm}
$$

The path of integration is raised or lowered a distance $\mu$ depending on whether $z_{ \pm}>0$ or $z_{ \pm}<0$, respectively, so as to make the integral $O\left[\exp \left(-\mu\left|z_{ \pm}\right|\right)\right]$. With an error of this order, the integral evaluates to $2 \mathrm{i} \pi \operatorname{sign} z_{ \pm}$times the sum of the residues of the integrand at any poles passed over in deforming the path. Given the regularity of $f_{ \pm}\left(k_{ \pm}, m_{ \pm}\right)$for real $m_{ \pm}$, and provided that $\epsilon$ is small enough, $\mu$ may be chosen such that no complex singularity of $f_{ \pm}\left(k_{ \pm}, m_{ \pm}\right)$is passed over, if any, and the only pole to consider is (2.15).

This procedure picks the pole with imaginary part of the same sign as $z_{ \pm}$, namely

$$
m_{ \pm} \sim \mathrm{i} \frac{\epsilon}{N \sin \theta_{0}}\left|k_{ \pm}\right| \operatorname{sign} z_{ \pm}
$$

subject to the condition

$$
\operatorname{sign} k_{ \pm}= \pm \operatorname{sign} z_{ \pm}
$$

thereby allowing only one half of the arm of the cross to contribute to the radiation. Physically, this amounts to imposing $\boldsymbol{c}_{\mathrm{g}} \cdot \boldsymbol{x}>0$, with $\boldsymbol{x}=(x, z)$ the position, namely to selecting the part of the wavenumber curve such that the component of the group velocity along the direction of observation points outwards not inwards, consistent with figure 1 . The introduction of $\epsilon$ has fulfilled its use, and we may now let $\epsilon \rightarrow 0$, to get

$$
m_{ \pm}=0 \quad \text { with } \quad \operatorname{sign} k_{ \pm}= \pm \operatorname{sign} z_{ \pm} .
$$

An asymptotic expansion of the waves follows,

$$
\psi \sim-\mathrm{i} \frac{\exp \left(-\mathrm{i} \omega_{0} t\right)}{4 \pi N^{2} \sin \theta_{0} \cos \theta_{0}} \int_{0}^{ \pm \infty \operatorname{sign} z_{ \pm}} f_{ \pm}\left(k_{ \pm}, m_{ \pm}=0\right) \exp \left(\mathrm{i} k_{ \pm} x_{ \pm}\right) \frac{\mathrm{d} k_{ \pm}}{k_{ \pm}},
$$

valid in the far field as $\left|z_{ \pm}\right| \rightarrow \infty$. It was first obtained by Lighthill (1978, §4.10).

We are looking for an exact expression of the waves in the near field, at finite $\left|z_{ \pm}\right|$. For 
this, more restrictive assumptions must be made about the source, which is assumed to be of compact support of radius $a$, in the sense that $f(x, z)=0$ for $r>a$. The spectrum $f_{ \pm}\left(k_{ \pm}, m_{ \pm}\right)$, being an integral over a finite domain, is an analytic function of the complex wavenumbers $k_{ \pm}$and $m_{ \pm}$; for each of them its modulus decreases asymptotically along the real axis, is small for $\left|\operatorname{Re} k_{ \pm}\right| \gtrsim 1 / a$ or $\left|\operatorname{Re} m_{ \pm}\right| \gtrsim 1 / a$, and grows exponentially along the imaginary axis. Specifically, we have

$$
\left|f_{ \pm}\left(k_{ \pm}, m_{ \pm}\right)\right|<\exp \left[a\left(\left|\operatorname{Im} k_{ \pm}\right|+\left|\operatorname{Im} m_{ \pm}\right|\right)\right] \iint_{r<a}|f(x, z)| \mathrm{d} x \mathrm{~d} z .
$$

This bound allows the path of integration of the inner integral in (2.12) to be closed by a semi-circle at infinity in the half-plane where the imaginary part of $m_{ \pm}$is of the same sign as $z_{ \pm}$: by a straightforward extension of Jordan's lemma, the contribution of the semi-circle vanishes provided that $\left|z_{ \pm}\right|>a$, and the integral evaluates to $2 \mathrm{i} \pi \operatorname{sign} z_{ \pm}$ times the sum of the residues of the integrand at any poles with imaginary part of the same sign as $z_{ \pm}$.

Given the analyticity of $f_{ \pm}\left(k_{ \pm}, m_{ \pm}\right)$, the only pole to consider is, again, (2.16). The waves follow immediately, when both $\left|z_{+}\right|>a$ and $\left|z_{-}\right|>a$, as

$$
\psi=-\mathrm{i} \frac{\exp \left(-\mathrm{i} \omega_{0} t\right)}{4 \pi N^{2} \sin \theta_{0} \cos \theta_{0}} \sum_{ \pm} \int_{0}^{ \pm \infty \operatorname{sign} z_{ \pm}} f_{ \pm}\left(k_{ \pm}, m_{ \pm}=0\right) \exp \left(\mathrm{i} k_{ \pm} x_{ \pm}\right) \frac{\mathrm{d} k_{ \pm}}{k_{ \pm}}
$$

or equivalently

$$
\begin{aligned}
\psi=-\mathrm{i} \frac{\exp \left(-\mathrm{i} \omega_{0} t\right)}{4 \pi N^{2} \sin \theta_{0} \cos \theta_{0}} \sum_{ \pm} \int_{0}^{\infty} f_{ \pm}\left(k_{ \pm}= \pm \kappa \operatorname{sign} z_{ \pm},\right. & \left.m_{ \pm}=0\right) \\
& \times \exp \left( \pm \mathrm{i} \kappa x_{ \pm} \operatorname{sign} z_{ \pm}\right) \frac{\mathrm{d} \kappa}{\kappa}
\end{aligned}
$$

As a result, if four wave beams are defined as the contributions of the four half-arms of the wavenumber curve, then at any given location two beams are received, one for the half $\operatorname{sign} k_{+}=\operatorname{sign} z_{+}$of the arm $m_{+}=0$, and the other for the half $\operatorname{sign} k_{-}=-\operatorname{sign} z_{-}$ of the arm $m_{-}=0$. In the far field, as $\left|z_{ \pm}\right| \rightarrow \infty$ with $x_{ \pm}$fixed, the contribution of one arm $m_{ \pm}=0$ becomes dominant compared with that for the other arm $m_{\mp}=0$; then (2.19) is recovered and each beam turns into a half-arm of the St. Andrew's Cross shown in figure 2 and observed experimentally by Görtler (1943), Mowbray \& Rarity (1967), Sutherland et al. (1999) and Zhang et al. (2007), among others.

Conversely, the line $z_{+}=0$ is seen to separate the two beams $k_{+}<0$ and $k_{+}>0$ originating from the arm $m_{+}=0$ of the wavenumber curve, and the line $z_{-}=0$ the two beams $k_{-}<0$ and $k_{-}>0$ originating from the arm $m_{-}=0$. For each beam, (2.22) is ascertained to be valid from the distance $\left|z_{ \pm}\right|=a$ where the beam leaves the source behind, up to infinity. Being based on an upper bound (2.20), it can be valid closer to the source, depending on the exact form of $f_{ \pm}\left(k_{ \pm}, m_{ \pm}\right)$; this, however, can only be assessed on a case-by-case basis, as will be seen later in $\S 8.1$.

\subsection{Viscous case}

Viscosity acts as another equivalent way of setting which part of the wavenumber curve is received at which location. The wave equation becomes

$$
\left[\left(\frac{\partial}{\partial t}-v \nabla^{2}\right) \frac{\partial}{\partial t} \nabla^{2}+N^{2} \nabla_{\mathrm{h}}^{2}\right] \psi=q,
$$


with $v$ the kinematic viscosity; see for example Voisin (2003). The response to twodimensional monochromatic forcing becomes

$$
\psi=\frac{\exp \left(-\mathrm{i} \omega_{0} t\right)}{4 \pi^{2}} \iint \frac{f(k, m) \exp [\mathrm{i}(k x+m z)]}{\omega_{0}^{2} \kappa^{2}-N^{2} k^{2}+\mathrm{i} \omega_{0} \nu \kappa^{4}} \mathrm{~d} k \mathrm{~d} m .
$$

Lighthill (1978, §4.10) did not evaluate this integral directly. Instead, he calculated the shear-induced rate of energy dissipation along the rays of a plane internal wave (Lighthill 1978, §4.7), then deduced from it an exponential attenuation factor to be added inside the inviscid expansion (2.19).

We proceed from (2.24), on the assumption $\nu \kappa^{2} / N \ll 1$ of small viscous effects. The addition of viscosity transforms the dispersion relation (2.8) into

$$
B\left(\omega_{0}, \boldsymbol{k}\right)=N^{2}\left[m_{ \pm} m_{\mp}+\mathrm{i} \frac{\nu}{N}\left(k_{ \pm}^{2}+m_{ \pm}^{2}\right)^{2} \cos \theta_{0}\right]=0,
$$

with $m_{\mp}$ given by (2.14), thus providing a fourth-order equation for $m_{ \pm}$. To leading order in $\nu k_{ \pm}^{2} / N$, the pole (2.9) is displaced to

$$
m_{ \pm} \sim \pm \mathrm{i} \beta k_{ \pm}^{3}
$$

where

$$
\beta=\frac{v}{2 N \sin \theta_{0}}
$$

The above deformations of contour pick

$$
m_{ \pm} \sim \mathrm{i} \beta\left|k_{ \pm}^{3}\right| \operatorname{sign} z_{ \pm} \quad \text { with } \operatorname{sign} k_{ \pm}= \pm \operatorname{sign} z_{ \pm}
$$

yielding for a rapidly decreasing source the far-field expansion, as $\left|z_{ \pm}\right| \rightarrow \infty$,

$$
\begin{aligned}
\psi \sim-\mathrm{i} \frac{\exp \left(-\mathrm{i} \omega_{0} t\right)}{4 \pi N^{2} \sin \theta_{0} \cos \theta_{0}} \int_{0}^{ \pm \infty \operatorname{sign} z_{ \pm}} f_{ \pm}\left(k_{ \pm},\right. & \left.m_{ \pm}=\mathrm{i} \beta\left|k_{ \pm}^{3}\right| \operatorname{sign} z_{ \pm}\right) \\
& \times \exp \left(-\beta\left|k_{ \pm}^{3} z_{ \pm}\right|\right) \exp \left(\mathrm{i} k_{ \pm} x_{ \pm}\right) \frac{\mathrm{d} k_{ \pm}}{k_{ \pm}}
\end{aligned}
$$

and for a source of compact support the exact expression, valid when both $\left|z_{+}\right|>a$ and $\left|z_{-}\right|>a$,

$$
\begin{aligned}
\psi=-\mathrm{i} \frac{\exp \left(-\mathrm{i} \omega_{0} t\right)}{4 \pi N^{2} \sin \theta_{0} \cos \theta_{0}} \sum_{ \pm} \int_{0}^{ \pm \infty \operatorname{sign} z_{ \pm}} f_{ \pm}\left(k_{ \pm}, m_{ \pm}=\mathrm{i} \beta\left|k_{ \pm}^{3}\right| \operatorname{sign} z_{ \pm}\right) \\
\quad \times \exp \left(-\beta\left|k_{ \pm}^{3} z_{ \pm}\right|\right) \exp \left(\mathrm{i} k_{ \pm} x_{ \pm}\right) \frac{\mathrm{d} k_{ \pm}}{k_{ \pm}}
\end{aligned}
$$

or equivalently

$$
\begin{aligned}
& \psi=-\mathrm{i} \frac{\exp \left(-\mathrm{i} \omega_{0} t\right)}{4 \pi N^{2} \sin \theta_{0} \cos \theta_{0}} \sum_{ \pm} \int_{0}^{\infty} f_{ \pm}( k_{ \pm} \\
&\left.= \pm \kappa \operatorname{sign} z_{ \pm}, m_{ \pm}=\mathrm{i} \beta \kappa^{3} \operatorname{sign} z_{ \pm}\right) \\
& \times \exp \left(-\beta \kappa^{3}\left|z_{ \pm}\right|\right) \exp \left( \pm \mathrm{i} \kappa x_{ \pm} \operatorname{sign} z_{ \pm}\right) \frac{\mathrm{d} \kappa}{\kappa}
\end{aligned}
$$

A new difference arises with Lighthill's (1978, §4.10) far-field analysis, in addition to the superposition of two wave beams at any given location; namely, the occurrence of the viscous correction (2.28) to the wavenumber not only for the propagation of the waves, as an attenuation factor, but also for their generation, inside the source spectrum. The relevance of this correction will be discussed later in $\S 8.1$. 


\subsection{Validity}

The preceding analysis is essentially a reformulation of the far-field expansion of Lighthill (1978, §4.10) for rapidly decreasing sources, and its extension to the near field for the smaller class of sources of compact support. The solutions of Hurley (1997) and Hurley \& Keady (1997) for the oscillations of an elliptic cylinder are of the type anticipated by Lighthill. When the cylinder is circular, the experiments of Sutherland et al. (1999) and Zhang et al. (2007) have confirmed their quantitative validity not only in the far field but also in the near field. When the cylinder is elliptic, the comparison with the experiments of Sutherland \& Linden (2002) has been less conclusive. The wave structure for the circular cylinder is illustrated in figure 3(a), where both beam separation and viscous attenuation are set by the along-beam coordinates $z_{ \pm}$.

The same is not true of all sources though: when the source is infinitely thin, beam separation and viscous attenuation are set by the normal coordinate to the source, $z$ for the horizontal segment in figure 3(b). Adapting the results of Tilgner (2000), Bardakov et al. (2007), Davis \& Llewellyn Smith (2010) and Le Dizès (2015) for the oscillations of a horizontal circular disc to the line source $q=g(x) \delta(z) \exp \left(-i \omega_{0} t\right)$, with $\delta$ the Dirac delta function, we obtain

$$
\begin{array}{rl}
\psi=-\mathrm{i} \frac{\exp \left(-\mathrm{i} \omega_{0} t\right)}{4 \pi N^{2} \sin \theta_{0} \cos \theta_{0}} \sum_{ \pm} \int_{0}^{ \pm \infty \operatorname{sign} z} & g\left(k=k_{ \pm} \cos \theta_{0}\right) \\
& \times \exp \left(-\beta\left|k_{ \pm}^{3} z\right| / \cos \theta_{0}\right) \exp \left(\mathrm{i} k_{ \pm} x_{ \pm}\right) \frac{\mathrm{d} k_{ \pm}}{k_{ \pm}}
\end{array}
$$

or equivalently

$$
\begin{aligned}
\psi=-\mathrm{i} \frac{\exp \left(-\mathrm{i} \omega_{0} t\right)}{4 \pi N^{2} \sin \theta_{0} \cos \theta_{0}} \sum_{ \pm} \int_{0}^{\infty} & g\left(k= \pm \kappa \cos \theta_{0} \operatorname{sign} z\right) \\
& \times \exp \left(-\beta \kappa^{3}|z| / \cos \theta_{0}\right) \exp \left( \pm \mathrm{i} \kappa x_{ \pm} \operatorname{sign} z\right) \frac{\mathrm{d} \kappa}{\kappa}
\end{aligned}
$$

At first glance the two wave structures, shown in figures $3(a, b)$, appear incompatible with each other. In particular, the spectrum $f(k, m)=g(k)$ of the line source leaves the normal wavenumber $m$ arbitrary, thereby allowing $\left|k_{ \pm}\right|$to become infinitely large and preventing the pole displacements (2.15) and (2.26) from remaining small, however small $\epsilon / N$ and $\beta \kappa^{2}$ can be.

In order to elucidate the effect of the geometry of the source on its wave radiation, we consider a source of elliptic shape in the following. The anticipated wave structure is illustrated in figure 3(c) for an elliptic cylinder, with both beam separation and viscous attenuation set by the normal distance to the line joining the two critical points where critical wave rays are tangential to the cylinder on either side. After a brief derivation of the wave equation in the following section, we will move on to the determination of the fluid velocity.

\section{Wave equation}

We consider a viscous uniformly stratified Boussinesq fluid of buoyancy frequency $N=-\left[\left(g / \rho_{0}\right)\left(\mathrm{d} \rho_{0} / \mathrm{d} z\right)\right]^{1 / 2}$ and kinematic viscosity $\nu$, having density distribution $\rho_{0}(z)$ and pressure distribution $p_{0}(z)$ at rest, related by the hydrostatic balance equation $\mathrm{d} p_{0} / \mathrm{d} z=-g \rho_{0}$, where $z$ is the upward vertical coordinate and $g$ the acceleration due to gravity. The linearized equations of motion for the density disturbance $\rho$, the pressure 

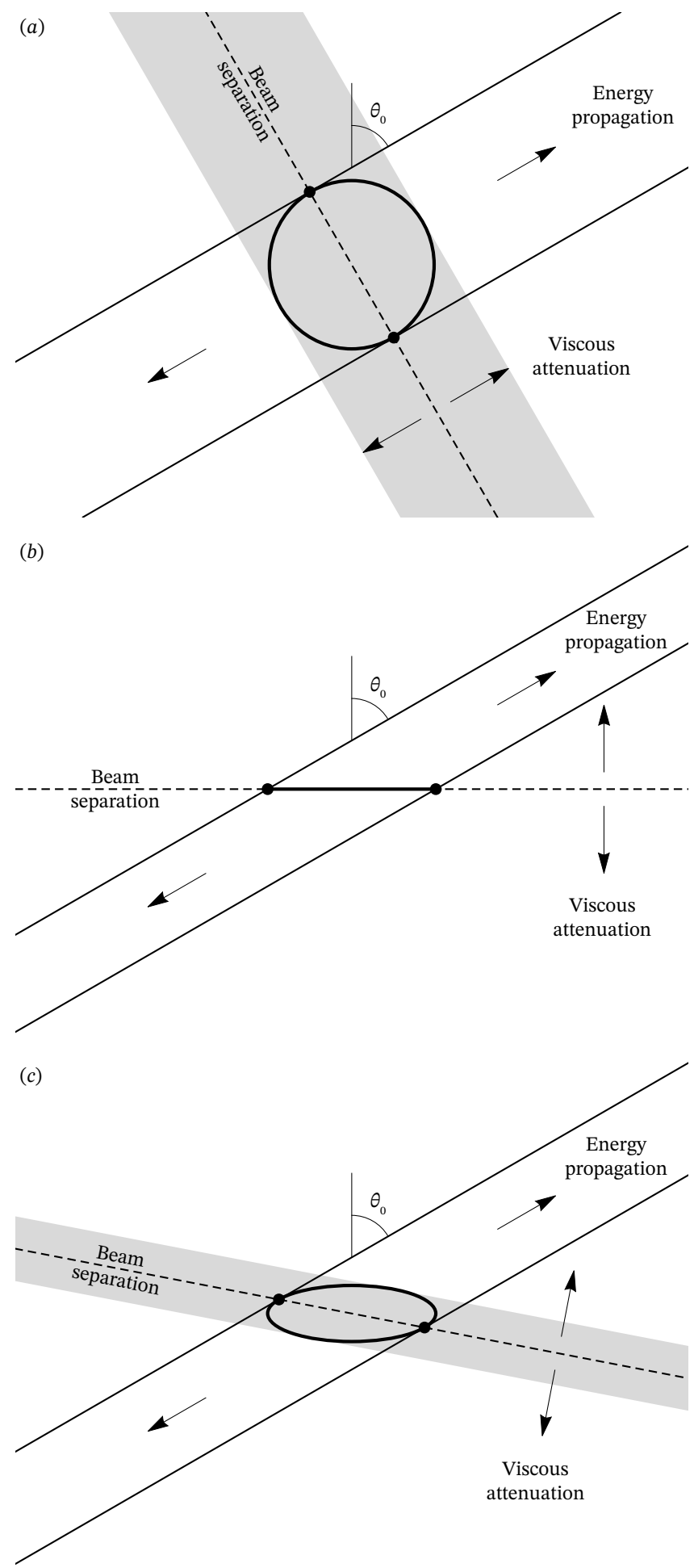

FIGURE 3. Structure of the beams propagating upward to the right and downward to the left, for $(a)$ a circular cylinder, $(b)$ a horizontal segment and $(c)$ an elliptic cylinder. The beams are delimited by the critical rays grazing the oscillating body at critical points on either side. The grey areas represent the zones where the validity of the theory is not ascertained. 
disturbance $p$ and the velocity $\boldsymbol{u}$ are

$$
\begin{gathered}
\rho_{0} \frac{\partial \boldsymbol{u}}{\partial t}=-\nabla p+\rho g \boldsymbol{e}_{z}+\rho_{0} \nu \nabla^{2} \boldsymbol{u}, \\
\boldsymbol{\nabla} \cdot \boldsymbol{u}=q, \\
\frac{\partial \rho}{\partial t}=\rho_{0} \frac{N^{2}}{g} w,
\end{gathered}
$$

respectively the Navier-Stokes equation, the equation of continuity, and the incompressible equation of state $\mathrm{d}\left(\rho_{0}+\rho\right) / \mathrm{d} t=0$. The source of the waves is modelled as a source of mass releasing the volume $q$ of fluid per unit volume per unit time.

The combination of these equations yields a single equation for the velocity,

$$
\left[\left(\frac{\partial}{\partial t}-v \nabla^{2}\right) \frac{\partial}{\partial t} \nabla^{2}+N^{2} \nabla_{\mathrm{h}}^{2}\right] \boldsymbol{u}=\left[\left(\frac{\partial}{\partial t}-v \nabla^{2}\right) \frac{\partial}{\partial t} \boldsymbol{\nabla}+N^{2} \nabla_{\mathrm{h}}\right] q,
$$

which will be our wave equation of choice in the following. For a two-dimensional monochromatic source $q=f(x, z) \exp \left(-i \omega_{0} t\right)$ of frequency $\omega_{0}<N$, introducing Fourier transforms according to (2.2), the velocity follows as

$$
\begin{aligned}
\boldsymbol{u}=\mathrm{i} \frac{\exp \left(-\mathrm{i} \omega_{0} t\right)}{4 \pi^{2}} \iint \frac{k \boldsymbol{e}_{x} \sin ^{2} \theta_{0}-m \boldsymbol{e}_{z} \cos ^{2} \theta_{0}-\mathrm{i}\left(\nu \kappa^{2} / N\right) \boldsymbol{k} \cos \theta_{0}}{m^{2} \cos ^{2} \theta_{0}-k^{2} \sin ^{2} \theta_{0}+\mathrm{i}\left(\nu \kappa^{4} / N\right) \cos \theta_{0}} \\
\quad \times f(k, m) \exp [\mathrm{i}(k x+m z)] \mathrm{d} k \mathrm{~d} m,
\end{aligned}
$$

where $\theta_{0}=\arccos \left(\omega_{0} / N\right)$ is the direction of propagation of the waves and $\kappa=|\boldsymbol{k}|$ the modulus of the wavenumber vector $\boldsymbol{k}=(k, m)$.

\section{Bluff forcing}

We consider a source of elliptic shape, having principal directions inclined at the angles $\varphi_{0}$ and $\varphi_{0}+\pi / 2$ to the $x$-axis, with $-\pi / 2<\varphi_{0} \leq \pi / 2$, and semi-axes $a$ and $b$, respectively. Introducing coordinates $\left(x_{0}, z_{0}\right)$ such that

$$
x_{0}=x \cos \varphi_{0}+z \sin \varphi_{0}, \quad z_{0}=-x \sin \varphi_{0}+z \cos \varphi_{0},
$$

the source satisfies $f_{0}\left(x_{0}, z_{0}\right)=f(x, z)=0$ for $x_{0}^{2} / a^{2}+z_{0}^{2} / b^{2}>1$. The characteristic coordinates (2.6) become

$$
x_{ \pm}=x_{0} \cos \left(\theta_{0} \pm \varphi_{0}\right) \mp z_{0} \sin \left(\theta_{0} \pm \varphi_{0}\right), \quad z_{ \pm}= \pm x_{0} \sin \left(\theta_{0} \pm \varphi_{0}\right)+z_{0} \cos \left(\theta_{0} \pm \varphi_{0}\right),
$$

and similarly for the wavenumbers (2.7). The velocity (3.5) becomes

$$
\begin{aligned}
\boldsymbol{u}=\mathrm{i} \frac{\exp \left(-\mathrm{i} \omega_{0} t\right)}{4 \pi^{2}} \iint \frac{k \boldsymbol{e}_{x} \sin ^{2} \theta_{0}-}{m^{2} \cos ^{2} \theta_{0}-\cos ^{2} \theta_{0}-\mathrm{i}\left(\nu \kappa^{2} / N\right) \boldsymbol{k} \cos \theta_{0} \theta_{0}+\mathrm{i}\left(\nu \kappa^{4} / N\right) \cos \theta_{0}} \\
\times f_{0}\left(k_{0}, m_{0}\right) \exp \left[\mathrm{i}\left(k_{0} x_{0}+m_{0} z_{0}\right)\right] \mathrm{d} k_{0} \mathrm{~d} m_{0},
\end{aligned}
$$

where $f_{0}\left(k_{0}, m_{0}\right)=f(k, m)$. We rescale coordinates and wavenumbers according to

$$
\left(X_{0}, Z_{0}\right)=\left(x_{0} / a, z_{0} / b\right), \quad\left(K_{0}, M_{0}\right)=\left(k_{0} a, m_{0} b\right),
$$

so that $F_{0}\left(X_{0}, Z_{0}\right)=f_{0}\left(x_{0}, z_{0}\right)=0$ for $\left|\boldsymbol{X}_{0}\right|>1$. 


\subsection{Inviscid case}

To proceed further, we start with the inviscid case and introduce the lengths

$$
c_{ \pm}=\left[a^{2} \cos ^{2}\left(\theta_{0} \pm \varphi_{0}\right)+b^{2} \sin ^{2}\left(\theta_{0} \pm \varphi_{0}\right)\right]^{1 / 2},
$$

the angles $\Theta_{ \pm}$such that

$$
\cos \Theta_{ \pm}=\frac{a}{c_{ \pm}} \cos \left(\theta_{0} \pm \varphi_{0}\right), \quad \sin \Theta_{ \pm}=\frac{b}{c_{ \pm}} \sin \left(\theta_{0} \pm \varphi_{0}\right)
$$

and the rescaled characteristic coordinates

$$
X_{ \pm}=X_{0} \cos \Theta_{ \pm} \mp Z_{0} \sin \Theta_{ \pm}, \quad Z_{ \pm}= \pm X_{0} \sin \Theta_{ \pm}+Z_{0} \cos \Theta_{ \pm},
$$

and similarly $\left(K_{ \pm}, M_{ \pm}\right)$in the wavenumber plane. These quantities are best interpreted by considering an elliptic cylinder of semi-axes $a$ and $b$, illustrated in figure 4 . Then, as pointed by Hurley (1997), $c_{+}$and $c_{-}$are the half-widths of the wave beams delimited by the critical rays tangential to the cylinder on either side.

The dispersion relation (2.8) becomes

$$
B\left(\omega_{0}, \boldsymbol{k}\right)=N^{2} \frac{c_{+} c_{-}}{a^{2} b^{2}} M_{+} M_{-}=0,
$$

implying that the wavenumber curve is still a St. Andrew's Cross but its arms now make different angles to the horizontal, $\Theta_{+}$for the arm $M_{+}=0$ and $\Theta_{-}$for the arm $M_{-}=0$. Proceeding as in $\S 2$, we evaluate the contribution of each arm in coordinates $\left(K_{ \pm}, M_{ \pm}\right)$, allowing $M_{ \pm}$to become complex and applying the residue theorem. The radiation condition transforms the dispersion relation into

$$
B\left(\omega_{0}+\mathrm{i} \epsilon, \boldsymbol{k}\right) \sim N^{2}\left(\frac{c_{+} c_{-}}{a^{2} b^{2}} M_{ \pm} M_{\mp}+2 \mathrm{i} \frac{\epsilon}{N} \kappa^{2} \cos \theta_{0}\right)=0,
$$

where

$$
M_{\mp}=\mp K_{ \pm} \sin \left(\Theta_{+}+\Theta_{-}\right)+M_{ \pm} \cos \left(\Theta_{+}+\Theta_{-}\right) \text {, }
$$

and

$$
\kappa^{2}=\frac{1}{c_{ \pm}^{2}} K_{ \pm}^{2}+\left(\frac{\sin ^{2} \Theta_{ \pm}}{a^{2}}+\frac{\cos ^{2} \Theta_{ \pm}}{b^{2}}\right) M_{ \pm}^{2} \pm 2\left(\frac{1}{a^{2}}-\frac{1}{b^{2}}\right) K_{ \pm} M_{ \pm} \sin \Theta_{ \pm} \cos \Theta_{ \pm},
$$

displacing the pole $M_{ \pm}=0$ to

$$
M_{ \pm} \sim \pm \mathrm{i} \frac{a b}{c_{ \pm}^{2}} \frac{\epsilon}{N \sin \theta_{0}} K_{ \pm} .
$$

Now, the spectrum $F_{ \pm}\left(K_{ \pm}, M_{ \pm}\right)=F_{0}\left(K_{0}, M_{0}\right)=f_{0}\left(k_{0}, m_{0}\right)$ is an analytic function of the complex wavenumbers $K_{ \pm}$and $M_{ \pm}$, for each decreasing asymptotically along the real axis, small for $\left|\operatorname{Re} K_{ \pm}\right| \gtrsim 1$ or $\left|\operatorname{Re} M_{ \pm}\right| \gtrsim 1$, and growing exponentially along the imaginary axis, with

$$
\left|F_{ \pm}\left(K_{ \pm}, M_{ \pm}\right)\right|<\exp \left(\left|\operatorname{Im} K_{ \pm}\right|+\left|\operatorname{Im} M_{ \pm}\right|\right) \iint_{\left|X_{0}\right|<1}\left|F_{0}\left(X_{0}, Z_{0}\right)\right| \mathrm{d} X_{0} \mathrm{~d} Z_{0} .
$$

Accordingly, the real path of integration for the variable $M_{ \pm}$may be closed by a semicircle at infinity in the half-plane where the imaginary part of $M_{ \pm}$is of the same sign as $Z_{ \pm}$, in such a way that the contribution of the semi-circle vanishes for $\left|Z_{ \pm}\right|>1$. The integral follows as $2 \mathrm{i} \pi \operatorname{sign} Z_{ \pm}$times the sum of the residues of the integrand at any poles 

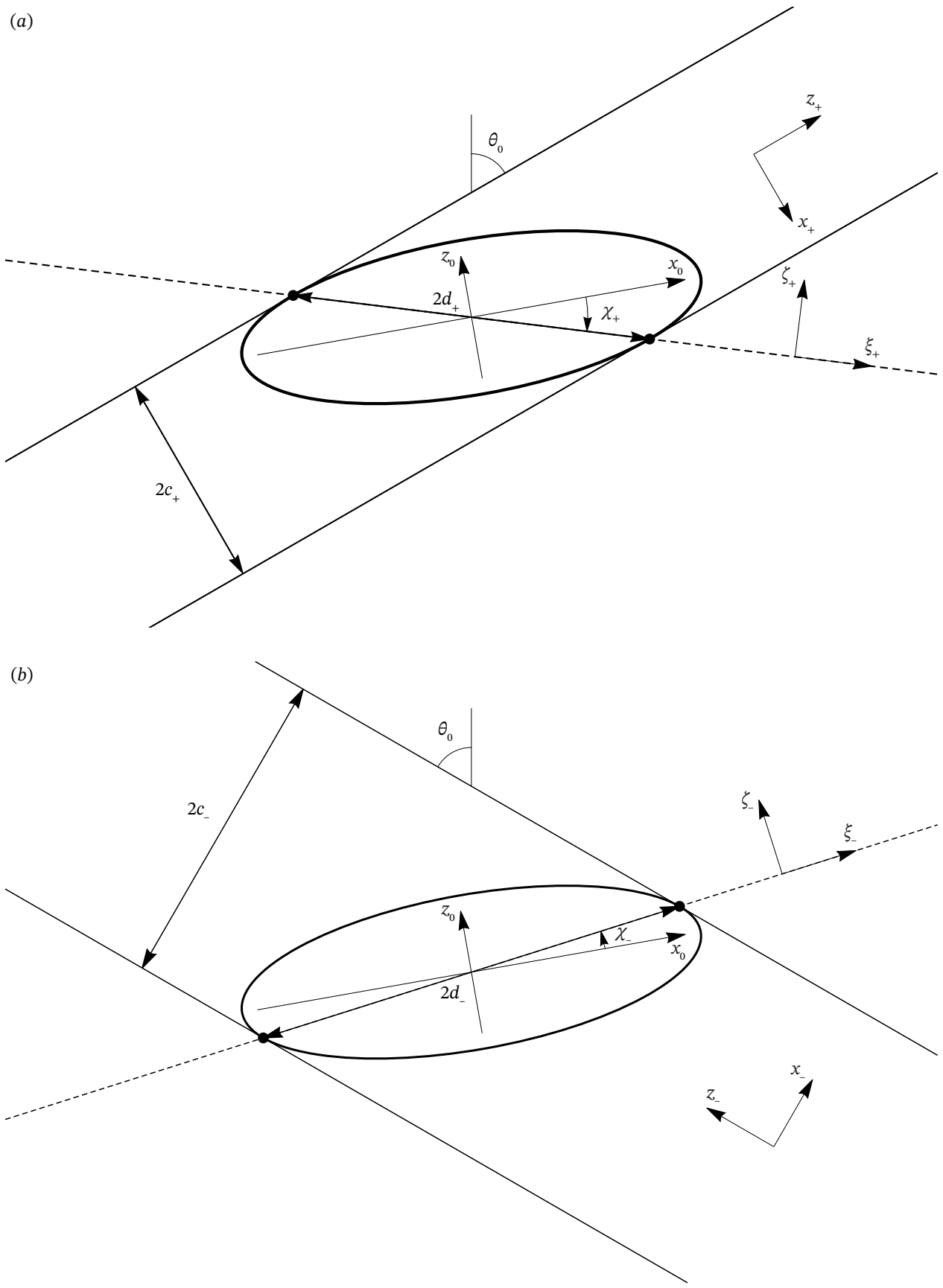

FIGURE 4. Geometry of the beams propagating $(a)$ upward to the right and downward to the left, and (b) upward to the left and downward to the right, for the oscillations of an elliptic cylinder.

with imaginary part of the same sign as $Z_{ \pm}$. This procedure picks the pole

$$
M_{ \pm} \sim \mathrm{i} \frac{a b}{c_{ \pm}^{2}} \frac{\epsilon}{N \sin \theta_{0}}\left|K_{ \pm}\right| \operatorname{sign} Z_{ \pm} \quad \text { with } \quad \operatorname{sign} K_{ \pm}= \pm \operatorname{sign} Z_{ \pm} .
$$

With $\epsilon / N \ll 1$ and $\left|K_{ \pm}\right|=O(1)$, the displacement is small and the limit $\epsilon \rightarrow 0$ may be 
applied, so that

$$
M_{ \pm}=0 \quad \text { with } \operatorname{sign} K_{ \pm}= \pm \operatorname{sign} Z_{ \pm} .
$$

The evaluation of the waves is then immediate when $\left|Z_{+}\right|>1$ and $\left|Z_{-}\right|>1$, yielding

$$
\boldsymbol{u}=\frac{\exp \left(-\mathrm{i} \omega_{0} t\right)}{4 \pi} \sum_{ \pm}( \pm) \frac{\boldsymbol{e}_{z_{ \pm}}}{c_{ \pm}} \int_{0}^{ \pm \infty \operatorname{sign} Z_{ \pm}} F_{ \pm}\left(K_{ \pm}, M_{ \pm}=0\right) \exp \left(\mathrm{i} K_{ \pm} X_{ \pm}\right) \mathrm{d} K_{ \pm} .
$$

To go back to unscaled coordinates, we introduce the new lengths

$$
d_{ \pm}=\left(a^{2} \cos ^{2} \Theta_{ \pm}+b^{2} \sin ^{2} \Theta_{ \pm}\right)^{1 / 2}=\frac{\left[a^{4} \cos ^{2}\left(\theta_{0} \pm \varphi_{0}\right)+b^{4} \sin ^{2}\left(\theta_{0} \pm \varphi_{0}\right)\right]^{1 / 2}}{c_{ \pm}},
$$

the new angles $\chi_{ \pm}$such that

$$
\begin{aligned}
& \cos \chi_{ \pm}=\frac{a}{d_{ \pm}} \cos \Theta_{ \pm}=\frac{a^{2}}{c_{ \pm} d_{ \pm}} \cos \left(\theta_{0} \pm \varphi_{0}\right), \\
& \sin \chi_{ \pm}=\frac{b}{d_{ \pm}} \sin \Theta_{ \pm}=\frac{b^{2}}{c_{ \pm} d_{ \pm}} \sin \left(\theta_{0} \pm \varphi_{0}\right),
\end{aligned}
$$

and the new coordinates

$$
\xi_{ \pm}=x_{0} \cos \chi_{ \pm} \mp z_{0} \sin \chi_{ \pm}, \quad \zeta_{ \pm}= \pm x_{0} \sin \chi_{ \pm}+z_{0} \cos \chi_{ \pm},
$$

and similarly $\left(\kappa_{ \pm}, \mu_{ \pm}\right)$in the wavenumber plane. For the elliptic cylinder, $d_{+}$and $d_{-}$ are the half-lengths of the critical segments joining the critical points where the critical rays are tangential to the cylinder on either side, and $\chi_{+}$and $\chi_{-}$are the angles of these segments to the positive $x_{0}$-axis, counted clockwise for $\chi_{+}$and counterclockwise for $\chi_{-}$, as shown in figure 4.

We have

$$
X_{ \pm}=\frac{x_{ \pm}}{c_{ \pm}}, \quad Z_{ \pm}=\zeta_{ \pm} \frac{d_{ \pm}}{a b}, \quad K_{ \pm}=\kappa_{ \pm} d_{ \pm}, \quad M_{ \pm}=m_{ \pm} \frac{a b}{c_{ \pm}} .
$$

Further, on the wavenumber curve $M_{ \pm}=0$, we also have $m_{ \pm}=0$ and $K_{ \pm}=k_{ \pm} c_{ \pm}$. It then follows that, when both $\left|\zeta_{+}\right|>a b / d_{+}$and $\left|\zeta_{-}\right|>a b / d_{-}$,

$$
\begin{aligned}
\boldsymbol{u}=\frac{\exp \left(-\mathrm{i} \omega_{0} t\right)}{4 \pi} \sum_{ \pm} \boldsymbol{e}_{z_{ \pm}} \operatorname{sign} \zeta_{ \pm} \int_{0}^{\infty} f_{ \pm}\left(k_{ \pm}= \pm \kappa \operatorname{sign} \zeta_{ \pm}, m_{ \pm}=0\right) \\
\times \exp \left( \pm \mathrm{i} \kappa x_{ \pm} \operatorname{sign} \zeta_{ \pm}\right) \mathrm{d} \kappa
\end{aligned}
$$

where $\kappa=\left|k_{ \pm}\right|=\left|K_{ \pm}\right| / c_{ \pm}$. Consistent with figure $3(c)$, beam separation is set by the coordinates $\zeta_{ \pm}$normal to the critical lines.

\subsection{Viscous case}

The presence of viscosity transforms the dispersion relation (4.8) into

$$
B\left(\omega_{0}, \boldsymbol{k}\right)=N^{2}\left(\frac{c_{+} c_{-}}{a^{2} b^{2}} M_{ \pm} M_{\mp}+\mathrm{i} \frac{\nu}{N} \kappa^{4} \cos \theta_{0}\right)=0 .
$$

There are now four poles, whose contributions are evaluated in the small-viscosity limit $N c_{+} c_{-} / \nu \gg 1$, corresponding to large Stokes number.

Two poles are associated with waves,

$$
M_{ \pm} \sim \pm \mathrm{i} \beta \frac{a b}{c_{ \pm}^{4}} K_{ \pm}^{3}
$$


where $\beta$ has been defined in (2.27). Closing the path of integration as above picks the pole

$$
M_{ \pm} \sim \mathrm{i} \beta \frac{a b}{c_{ \pm}^{4}}\left|K_{ \pm}\right|^{3} \operatorname{sign} Z_{ \pm} \quad \text { with } \quad \operatorname{sign} K_{ \pm}= \pm \operatorname{sign} Z_{ \pm} .
$$

The assumptions $\beta / c_{ \pm}^{2} \ll 1$ and $\left|K_{ \pm}\right|=O(1)$ ensure its smallness. Setting it to zero in the slowly varying rational fraction in the integrand of (4.3) when evaluating the residue, but keeping it nonzero in the spectrum and in the rapidly varying complex exponential, the velocity follows for $\left|Z_{+}\right|>1$ and $\left|Z_{-}\right|>1$ as

$$
\begin{aligned}
\boldsymbol{u}=\frac{\exp \left(-\mathrm{i} \omega_{0} t\right)}{4 \pi} \sum_{ \pm}( \pm) \frac{\boldsymbol{e}_{z_{ \pm}}}{c_{ \pm}} \int_{0}^{ \pm \infty \operatorname{sign} Z_{ \pm}} & F_{ \pm}\left(K_{ \pm}, M_{ \pm}=\mathrm{i} \beta \frac{a b}{c_{ \pm}^{4}}\left|K_{ \pm}\right|^{3} \operatorname{sign} Z_{ \pm}\right) \\
& \times \exp \left(-\beta \frac{a b}{c_{ \pm}^{4}}\left|K_{ \pm}^{3} Z_{ \pm}\right|\right) \exp \left(\mathrm{i} K_{ \pm} X_{ \pm}\right) \mathrm{d} K_{ \pm}
\end{aligned}
$$

that is in unscaled coordinates, for $\left|\zeta_{+}\right|>a b / d_{+}$and $\left|\zeta_{-}\right|>a b / d_{-}$,

$$
\begin{aligned}
\boldsymbol{u}=\frac{\exp \left(-\mathrm{i} \omega_{0} t\right)}{4 \pi} \sum_{ \pm} \boldsymbol{e}_{z_{ \pm}} \operatorname{sign} \zeta_{ \pm} \int_{0}^{\infty} & F_{ \pm}\left(K_{ \pm}= \pm \kappa c_{ \pm} \operatorname{sign} \zeta_{ \pm}, M_{ \pm}=\mathrm{i} \beta \kappa^{3} \frac{a b}{c_{ \pm}} \operatorname{sign} \zeta_{ \pm}\right) \\
& \times \exp \left(-\beta \kappa^{3} \frac{d_{ \pm}}{c_{ \pm}}\left|\zeta_{ \pm}\right|\right) \exp \left( \pm \mathrm{i} \kappa x_{ \pm} \operatorname{sign} \zeta_{ \pm}\right) \mathrm{d} \kappa
\end{aligned}
$$

where $\kappa=\left|\operatorname{Re} k_{ \pm}\right|=\left|K_{ \pm}\right| / c_{ \pm}$and

$$
\frac{d_{ \pm}}{c_{ \pm}} \zeta_{ \pm}= \pm \frac{b^{2}}{c_{ \pm}^{2}} x_{0} \sin \left(\theta_{0} \pm \varphi_{0}\right)+\frac{a^{2}}{c_{ \pm}^{2}} z_{0} \cos \left(\theta_{0} \pm \varphi_{0}\right)
$$

Consistent with figure 3(c), viscous attenuation is set by $\zeta_{ \pm}$. When the source is circular $(a=b)$, we have $c_{ \pm}=d_{ \pm}=a$ and $\chi_{ \pm}=\theta_{0} \pm \varphi_{0}$, so that $\zeta_{ \pm}=z_{ \pm}$and the pattern in figure $3(a)$ is recovered; when the source is a horizontal segment $\left(b=0\right.$ and $\left.\varphi_{0}=0\right)$, we have $c_{ \pm}=a \cos \theta_{0}$ and $d_{ \pm}=a$, and also $\chi_{ \pm}=0$, so that $\zeta_{ \pm}=z$ and the pattern in figure $3(b)$ is recovered.

The other two poles are associated with boundary layers along the lines $Z_{ \pm}=0$, hence $\zeta_{ \pm}=0$. To leading order they satisfy

$$
M_{ \pm}^{2} \sim \mathrm{i} \frac{N c_{+} c_{-}}{v} \frac{a^{2} b^{2}}{d_{ \pm}^{4}} \frac{\cos \left(\Theta_{+}+\Theta_{-}\right)}{\cos \theta_{0}}
$$

which combined with the condition $\left|Z_{ \pm}\right|>1$ means that their contributions are

$$
O\left\{\exp \left[-\left(\frac{N c_{+} c_{-}}{v}\right)^{1 / 2} \frac{a b}{d_{ \pm}^{2}}\left|\frac{\cos \left(\Theta_{+}+\Theta_{-}\right)}{\cos \theta_{0}}\right|^{1 / 2}\right]\right\} .
$$

Given the small-viscosity assumption $N c_{+} c_{-} / \nu \gg 1$, the only way for these contributions to be significant, apart from the pathological case

$$
a^{2} \cos \left(\theta_{0}+\varphi_{0}\right) \cos \left(\theta_{0}-\varphi_{0}\right)=b^{2} \sin \left(\theta_{0}+\varphi_{0}\right) \sin \left(\theta_{0}-\varphi_{0}\right)
$$

corresponding to $\cos \left(\Theta_{+}+\Theta_{-}\right)=0$, is that $a=0$ or $b=0$, namely that the source be infinitely thin. Accordingly, consistent with physical intuition, no boundary layer forms for bluff forcing of nonzero $a$ and $b$, and for line forcing the boundary layer forms along the line itself. 


\section{Line forcing}

We consider line forcing separately in this section. Without loss of generality, the source is assumed to have finite and nonzero size $2 a$ along the $x_{0}$-axis, and zero size along the $z_{0}$-axis. Forcing is assumed to be inviscid, with implications discussed later in $§ 5.3$. Then, using (3.2), (3.3) and the inviscid version of (3.1), either the pressure is prescribed on both sides of the line $z_{0}=0$ yielding a velocity discontinuity $2 w_{0}\left(x_{0}\right)$ across it, so that

$$
f_{0}\left(x_{0}, z_{0}\right)=2 w_{0}\left(x_{0}\right) \delta\left(z_{0}\right),
$$

or the velocity is prescribed yielding a pressure discontinuity $2 p_{0}\left(x_{0}\right)$, so that

$$
f_{0}\left(x_{0}, z_{0}\right)=2 \mathrm{i} \frac{\cos \left(\theta_{0}+\varphi_{0}\right) \cos \left(\theta_{0}-\varphi_{0}\right) p_{0}\left(x_{0}\right) \delta^{\prime}\left(z_{0}\right)+\sin \varphi_{0} \cos \varphi_{0} p_{0}^{\prime}\left(x_{0}\right) \delta\left(z_{0}\right)}{\rho_{0} \omega_{0} \sin ^{2} \theta_{0}} .
$$

For a horizontal disc, Gabov \& Pletner (1988) considered the former forcing and Martin \& Llewellyn Smith $(2011,2012 b)$ the latter. We take

$$
f_{0}\left(x_{0}, z_{0}\right)=g\left(x_{0}\right) \delta^{(n)}\left(z_{0}\right), \quad f_{0}\left(k_{0}, m_{0}\right)=g\left(k_{0}\right)\left(\mathrm{i} m_{0}\right)^{n},
$$

where $\delta^{(n)}$ is the $n$-th derivative of the Dirac delta function, with $n=0$ or 1 . The function $g\left(x_{0}\right)$ is assumed to decrease rapidly for $\left|x_{0}\right| \gtrsim a$, so that its spectrum $g\left(k_{0}\right)$ is appreciable only for $\left|k_{0}\right| \lesssim 1 / a$ and small at larger $\left|k_{0}\right|$. This assumption, less restrictive than the compact support assumption, leaves the possibility for the forcing to be Gaussian, as in $\S 8.4$ below.

We follow the approach introduced by Kistovich \& Chashechkin $(1994,1995)$ for the reflection of the wave beam from a point source at an inclined plane, and Chashechkin \& Kistovich (1997) and Kistovich \& Chashechkin $(1999 a, b)$ for the generation of wave beams by the oscillations of an inclined plate; namely, we proceed in coordinates $\left(x_{0}, z_{0}\right)$, applying the residue theorem to integration over $m_{0}$ and dealing directly with the viscous case in the small-viscosity limit $N a^{2} / \nu \gg 1$.

\subsection{Inclined source}

The dispersion relation (2.25) writes

$$
B\left(\omega_{0}, \boldsymbol{k}\right)=N^{2}\left[m_{+} m_{-}+\mathrm{i} \frac{\nu}{N}\left(k_{0}^{2}+m_{0}^{2}\right)^{2} \cos \theta_{0}\right]=0
$$

where

$$
m_{ \pm}=k_{0} \sin \left(\varphi_{0} \pm \theta_{0}\right)+m_{0} \cos \left(\varphi_{0} \pm \theta_{0}\right)
$$

Two of its solutions are associated with waves,

$$
m_{0} \sim-k_{0} \tan \left(\varphi_{0} \pm \theta_{0}\right) \pm \mathrm{i} \frac{\beta k_{0}^{3}}{\cos ^{4}\left(\varphi_{0} \pm \theta_{0}\right)},
$$

the condition $\left|k_{0}\right| a=O(1)$ combined with the assumption $\beta / a^{2} \ll 1$ ensuring that they remain close to their inviscid values $m_{0} \sim-k_{0} \tan \left(\varphi_{0} \pm \theta_{0}\right)$. Closing the real path of integration by a semi-circle at infinity in the half-plane where the imaginary part of $m_{0}$ is of the same sign as $z_{0}$, we keep the solution

$$
m_{0} \sim-k_{0} \tan \left(\varphi_{0}+\theta_{0} \operatorname{sign} k_{0} \operatorname{sign} z_{0}\right)+\mathrm{i} \frac{\beta\left|k_{0}\right|^{3} \operatorname{sign} z_{0}}{\cos ^{4}\left(\varphi_{0}+\theta_{0} \operatorname{sign} k_{0} \operatorname{sign} z_{0}\right)},
$$


and obtain

$$
\begin{aligned}
\boldsymbol{u}= & \frac{\exp \left(-\mathrm{i} \omega_{0} t\right)}{4 \pi} \int_{-\infty}^{\infty} \frac{\boldsymbol{e}_{x} \sin \theta_{0} \operatorname{sign} k_{0}+\boldsymbol{e}_{z} \cos \theta_{0} \operatorname{sign} z_{0}}{\cos \left(\varphi_{0}+\theta_{0} \operatorname{sign} k_{0} \operatorname{sign} z_{0}\right)} g\left(k_{0}\right) \\
& \times\left[-\mathrm{i} k_{0} \tan \left(\varphi_{0}+\theta_{0} \operatorname{sign} k_{0} \operatorname{sign} z_{0}\right)\right]^{n} \exp \left[-\frac{\beta\left|k_{0}^{3} z_{0}\right|}{\cos ^{4}\left(\varphi_{0}+\theta_{0} \operatorname{sign} k_{0} \operatorname{sign} z_{0}\right)}\right] \\
& \times \exp \left\{\mathrm{i} k_{0}\left[x_{0}-z_{0} \tan \left(\varphi_{0}+\theta_{0} \operatorname{sign} k_{0} \operatorname{sign} z_{0}\right)\right]\right\} \mathrm{d} k_{0},
\end{aligned}
$$

or equivalently

$$
\begin{aligned}
\boldsymbol{u}= & \frac{\exp \left(-\mathrm{i} \omega_{0} t\right)}{4 \pi} \sum_{ \pm} \boldsymbol{e}_{z_{ \pm}}\left[-\mathrm{i} \sin \left(\theta_{0} \pm \varphi_{0}\right)\right]^{n}\left[\operatorname{sign} z_{0} \operatorname{sign} \cos \left(\theta_{0} \pm \varphi_{0}\right)\right]^{n+1} \\
& \times \int_{0}^{\infty} \kappa^{n} g\left[k_{0}= \pm \kappa\left|\cos \left(\theta_{0} \pm \varphi_{0}\right)\right| \operatorname{sign} z_{0}\right] \exp \left[-\frac{\beta \kappa^{3}\left|z_{0}\right|}{\left|\cos \left(\theta_{0} \pm \varphi_{0}\right)\right|}\right] \\
& \times \exp \left[ \pm \mathrm{i} \kappa x_{ \pm} \operatorname{sign} z_{0} \operatorname{sign} \cos \left(\theta_{0} \pm \varphi_{0}\right)\right] \mathrm{d} \kappa,
\end{aligned}
$$

where $\kappa=\left|\operatorname{Re} k_{ \pm}\right|=\left|k_{0}\right| /\left|\cos \left(\theta_{0} \pm \varphi_{0}\right)\right|$, consistent with (4.26).

The other two solutions are

$$
m_{0} \sim\left[\mathrm{i} \frac{N}{v} \frac{\cos \left(\theta_{0}+\varphi_{0}\right) \cos \left(\theta_{0}-\varphi_{0}\right)}{\cos \theta_{0}}\right]^{1 / 2}+k_{0} \frac{\sin \varphi_{0} \cos \varphi_{0}}{\cos \left(\theta_{0}+\varphi_{0}\right) \cos \left(\theta_{0}-\varphi_{0}\right)},
$$

where the undetermined square root in the first term can take either value. Closing the path of integration as above, we pick

$$
\begin{aligned}
m_{0} \sim k_{\mathrm{b}}\left[\mathrm{i}+\operatorname{sign} \cos \left(\theta_{0}+\varphi_{0}\right) \operatorname{sign} \cos \left(\theta_{0}-\varphi_{0}\right)\right] & \operatorname{sign} z_{0} \\
& +k_{0} \frac{\sin \varphi_{0} \cos \varphi_{0}}{\cos \left(\theta_{0}+\varphi_{0}\right) \cos \left(\theta_{0}-\varphi_{0}\right)}
\end{aligned}
$$

where

$$
k_{\mathrm{b}}=\left(\frac{\omega_{0}}{2 \nu}\right)^{1 / 2} \frac{\left|\cos \left(\theta_{0}+\varphi_{0}\right) \cos \left(\theta_{0}-\varphi_{0}\right)\right|^{1 / 2}}{\cos \theta_{0}} .
$$

The associated velocity disturbance is

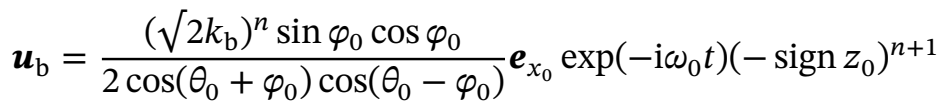

$$
\begin{aligned}
& \times g\left[x_{0}+z_{0} \frac{\sin \varphi_{0} \cos \varphi_{0}}{\cos \left(\theta_{0}+\varphi_{0}\right) \cos \left(\theta_{0}-\varphi_{0}\right)}\right] \exp \left(-k_{\mathrm{b}}\left|z_{0}\right|\right) \\
& \times \exp \left[\mathrm{i}\left(k_{\mathrm{b}}\left|z_{0}\right|-n \frac{\pi}{4}\right) \operatorname{sign} \cos \left(\theta_{0}+\varphi_{0}\right) \operatorname{sign} \cos \left(\theta_{0}-\varphi_{0}\right)\right],
\end{aligned}
$$

and corresponds to a boundary layer of thickness $1 / k_{\mathrm{b}}$, small compared with $a$, around the line $z_{0}=0$. The velocity within the layer is $O\left[\left(\mathrm{Na}^{2} / \nu\right)^{n / 2}\right]$ compared with that for the waves, hence of the same order for $n=0$ and large compared with it for $n=1$. As for the classical Stokes layer, the velocity is directed along the source, in the $x_{0}$-direction. Its variations combine transverse propagation at the velocity $\omega_{0} / k_{\mathrm{b}}$ in the $z_{0}$-direction, and reproduction of the longitudinal variations $g\left(x_{0}\right)$ imposed at the source, shifted in proportion to $z_{0}$. When the source is either horizontal $\left(\varphi_{0}=0\right)$ or vertical $\left(\varphi_{0}=\pi / 2\right)$, the leading-order term (5.13) vanishes and the expansion must be carried to the next order.

In the event $n=1$ that Jordan's lemma does not apply, a third contribution to the 
velocity is associated with the semi-circle closing the path of integration at infinity. This possibility is considered in Appendix A.

\subsection{Horizontal and vertical sources}

For a horizontal line source, we have $x_{0}=x$ and $z_{0}=z$. Separating the exponential transform $g(k)$ into cosine and sine transforms

$$
g^{(c)}(k)=\int g(x) \cos (k x) d x, \quad g^{(s)}(k)=\int g(x) \sin (k x) d x,
$$

the original source distribution $g(x)$ separates into even and odd parts, respectively, and we obtain for the waves

$$
\begin{aligned}
\boldsymbol{u}= & \frac{\exp \left(-\mathrm{i} \omega_{0} t\right)}{2 \pi}\left(-\mathrm{i} \sin \theta_{0} \operatorname{sign} z\right)^{n} \int_{0}^{\infty} \kappa^{n} \exp \left(-\beta \kappa^{3}|z| / \cos \theta_{0}\right) \exp \left(-\mathrm{i} \kappa|z| \sin \theta_{0}\right) \\
& \times\left\{g^{(\mathrm{c})}\left(k=\kappa \cos \theta_{0}\right)\left[\boldsymbol{e}_{z} \cos \theta_{0} \cos \left(\kappa x \cos \theta_{0}\right) \operatorname{sign} z+\mathrm{i} \boldsymbol{e}_{x} \sin \theta_{0} \sin \left(\kappa x \cos \theta_{0}\right)\right]\right. \\
& \left.+g^{(\mathrm{s})}\left(k=\kappa \cos \theta_{0}\right)\left[\boldsymbol{e}_{z} \cos \theta_{0} \sin \left(\kappa x \cos \theta_{0}\right) \operatorname{sign} z-\mathrm{i} \boldsymbol{e}_{x} \sin \theta_{0} \cos \left(\kappa x \cos \theta_{0}\right)\right]\right\} \mathrm{d} \kappa .
\end{aligned}
$$

The boundary layer is given by

$$
\begin{aligned}
\boldsymbol{u}_{\mathrm{b}}=-\frac{\left(\sqrt{2} k_{\mathrm{b}}\right)^{n-1}}{2 \cos ^{2} \theta_{0}} \boldsymbol{e}_{x} \exp \left(-\mathrm{i} \omega_{0} t\right)(- & \operatorname{sign} z)^{n} g^{\prime}(x) \\
& \times \exp \left(-k_{\mathrm{b}}|z|\right) \exp \left\{\mathrm{i}\left[k_{\mathrm{b}}|z|-(n-1) \frac{\pi}{4}\right]\right\},
\end{aligned}
$$

where

$$
k_{\mathrm{b}}=\left(\frac{\omega_{0}}{2 v}\right)^{1 / 2} .
$$

It is $O\left[\left(N a^{2} / \nu\right)^{(n-1) / 2}\right]$ compared with the waves, hence negligible for $n=0$ and of the same order for $n=1$. Its structure is the same as before, with longitudinal velocity and transverse propagation, except for the longitudinal variations which are the derivative $g^{\prime}(x)$ of those imposed at the source.

For a vertical line source, we have $x_{0}=z$ and $z_{0}=-x$. Introducing the cosine and sine transforms

$$
g^{(c)}(m)=\int g(z) \cos (m z) \mathrm{d} z, \quad g^{(\mathrm{s})}(m)=\int g(z) \sin (m z) \mathrm{d} z,
$$

we obtain

$$
\begin{aligned}
\boldsymbol{u}= & \frac{\exp \left(-\mathrm{i} \omega_{0} t\right)}{2 \pi}\left(-\mathrm{i} \cos \theta_{0} \operatorname{sign} x\right)^{n} \int_{0}^{\infty} \kappa^{n} \exp \left(-\beta \kappa^{3}|x| / \sin \theta_{0}\right) \exp \left(\mathrm{i} \kappa|x| \cos \theta_{0}\right) \\
& \times\left\{g^{(\mathrm{c})}\left(m=\kappa \sin \theta_{0}\right)\left[\boldsymbol{e}_{x} \sin \theta_{0} \cos \left(\kappa z \sin \theta_{0}\right) \operatorname{sign} x-\mathrm{i} \boldsymbol{e}_{z} \cos \theta_{0} \sin \left(\kappa z \sin \theta_{0}\right)\right]\right. \\
& \left.+g^{(\mathrm{s})}\left(m=\kappa \sin \theta_{0}\right)\left[\boldsymbol{e}_{x} \sin \theta_{0} \sin \left(\kappa z \sin \theta_{0}\right) \operatorname{sign} x+\mathrm{i} \boldsymbol{e}_{z} \cos \theta_{0} \cos \left(\kappa z \sin \theta_{0}\right)\right]\right\} \mathrm{d} \kappa .
\end{aligned}
$$

The boundary layer is given by

$$
\begin{aligned}
\boldsymbol{u}_{\mathrm{b}}=-\frac{\left(\sqrt{2 k_{\mathrm{b}}}\right)^{n-1}}{2 \sin ^{2} \theta_{0}} \boldsymbol{e}_{z} \exp \left(-\mathrm{i} \omega_{0} t\right)( & \operatorname{sign} x)^{n} g^{\prime}(z) \\
& \times \exp \left(-k_{\mathrm{b}}|x|\right) \exp \left\{-\mathrm{i}\left[k_{\mathrm{b}}|x|-(n-1) \frac{\pi}{4}\right]\right\},
\end{aligned}
$$


where

$$
k_{\mathrm{b}}=\left(\frac{\omega_{0}}{2 v}\right)^{1 / 2} \tan \theta_{0}
$$

implying the same structure as for a horizontal source.

\subsection{Relevance}

At this stage, no assumption has been made regarding the actual boundary condition at the source. The aim was to point that, in a viscous fluid, when forcing takes place at a line, in the form (5.3), the solution of the wave equation contains not only waves but also a boundary layer, and to highlight how stratification affects this layer. In particular, when the line is horizontal, the layer thickness, $1 / k_{\mathrm{b}}$ say, is the same penetration depth $\left(2 \nu / \omega_{0}\right)^{1 / 2}$ as in a homogeneous fluid according to (5.17); when the line is inclined, it varies with the angle of propagation of the waves according to (5.12), becoming (5.21) when the line is vertical.

In practice, the actual mechanism by which the waves are generated is the imposition of a no-slip condition at a rectilinear boundary. Accordingly, the representation of the forcing arises as a consequence of solving the full boundary-value problem, not as an ingredient of its formulation; in other words, the knowledge of the boundary layer is a prerequisite for the representation of the forcing, not the other way round. Consider a plate or a disc. When its oscillations are broadside, forcing becomes free-slip in the limit of large Stokes number $\omega_{0} a^{2} / \nu \gg 1$. For a horizontal disc, Davis \& Llewellyn Smith (2010) have shown that, in this limit, the force exerted on the disc approaches its inviscid value. In these circumstances, the forcing can be represented by a distribution of mass sources taken from inviscid (but stratified) flow theory. When the oscillations are edgewise, forcing is no-slip and wave generation is entirely attributable to viscosity. For a two-dimensional inclined plate, Chashechkin \& Kistovich (1997) and Kistovich \& Chashechkin $(1999 a, b)$ have considered the possibility of representing the plate by a distribution of force sources taken from homogeneous (but viscous) flow theory. They found that, although the waves and the boundary layer have identical structures for the force sources and for the actual boundary condition, their amplitudes are different, especially in the pathological cases $\varphi_{0}= \pm\left(\pi / 2-\theta_{0}\right)$.

The particular case of a two-dimensional horizontal boundary has been considered by Hurley \& Hood (2001) and Renaud \& Venaille (2019). When the same vertical velocity is imposed on both sides of the boundary, corresponding to the oscillations of a rigid plate, the present analysis for $n=1$ yields a boundary layer of the same order as the waves; the same conclusion has been reached by Hurley \& Hood (2001) using a free-slip boundary condition. When a given vertical velocity profile is imposed on part of an otherwise fixed boundary, as for the wave generator in $\$ 8.3$, and the image of the profile through this boundary is added, the present analysis for $n=0$ predicts that the boundary layer is negligible compared with the waves; for an undulating horizontal wall, the boundary layer has been found by Renaud \& Venaille (2019) to be negligible compared with the waves when a free-slip boundary condition is used, and of the same order as them when a no-slip condition is used.

\section{Alternative approaches}

It follows from the preceding sections that, for a given source function $f(x, z)$, different expressions of the waves may be obtained, depending on the direction along which the residue theorem is applied in the wavenumber plane. Each expression has a domain 
of validity set by the extent of the source along that direction, the various expressions becoming equivalent wherever their domains of validity intersect. We investigate some expressions in the present section.

\subsection{Inclined source}

Consider first the characteristic coordinates $\left(x_{ \pm}, z_{ \pm}\right)$as in $\S 2$, and integrate over $m_{ \pm}$to get

$$
\begin{aligned}
\boldsymbol{u}=\frac{\exp \left(-\mathrm{i} \omega_{0} t\right)}{4 \pi} \sum_{ \pm} \boldsymbol{e}_{z_{ \pm}} \operatorname{sign} z_{ \pm} \int_{0}^{\infty} f_{ \pm}\left(k_{ \pm}\right. & \left.= \pm \kappa \operatorname{sign} z_{ \pm}, m_{ \pm}=\mathrm{i} \beta \kappa^{3} \operatorname{sign} z_{ \pm}\right) \\
& \times \exp \left(-\beta \kappa^{3}\left|z_{ \pm}\right|\right) \exp \left( \pm \mathrm{i} \kappa x_{ \pm} \operatorname{sign} z_{ \pm}\right) \mathrm{d} \kappa
\end{aligned}
$$

If the support of the source is circular of radius $a$, such that $f_{ \pm}\left(x_{ \pm}, z_{ \pm}\right)=0$ for $\left|z_{ \pm}\right|>a$, this result is valid when both $\left|z_{+}\right|>a$ and $\left|z_{-}\right|>a$. If the support is elliptic of semi-axes $a$ and $b$, such that $f_{ \pm}\left(x_{ \pm}, z_{ \pm}\right)=0$ for $\left|z_{ \pm}\right|>\left[a^{2} \sin ^{2}\left(\theta_{0} \pm \varphi_{0}\right)^{2}+b^{2} \cos \left(\theta_{0} \pm \varphi_{0}\right)^{2}\right]^{1 / 2}$, the result becomes valid when both $\left|z_{+}\right|>\left[a^{2} \sin ^{2}\left(\theta_{0}+\varphi_{0}\right)^{2}+b^{2} \cos \left(\theta_{0}+\varphi_{0}\right)^{2}\right]^{1 / 2}$ and $\left|z_{-}\right|>\left[a^{2} \sin ^{2}\left(\theta_{0}-\varphi_{0}\right)^{2}+b^{2} \cos \left(\theta_{0}-\varphi_{0}\right)^{2}\right]^{1 / 2}$.

Alternatively, consider the original Cartesian coordinates $\left(x_{0}, z_{0}\right)$ as in $\S 5$, and integrate over $m_{0}$ to get

$$
\begin{aligned}
\boldsymbol{u}=\frac{\exp \left(-\mathrm{i} \omega_{0} t\right)}{4 \pi} & \operatorname{sign} z_{0} \sum_{ \pm} \boldsymbol{e}_{z_{ \pm}} \operatorname{sign} \cos \left(\theta_{0} \pm \varphi_{0}\right) \int_{0}^{\infty} f_{0}\left(k_{0}=k_{0}^{\prime}, m_{0}=m_{0}^{\prime}\right) \\
& \times \exp \left[-\frac{\beta \kappa^{3}\left|z_{0}\right|}{\left|\cos \left(\theta_{0} \pm \varphi_{0}\right)\right|}\right] \exp \left[ \pm \mathrm{i} \kappa x_{ \pm} \operatorname{sign} \cos \left(\theta_{0} \pm \varphi_{0}\right) \operatorname{sign} z_{0}\right] \mathrm{d} \kappa
\end{aligned}
$$

where

$$
\begin{gathered}
k_{0}^{\prime}= \pm \kappa\left|\cos \left(\theta_{0} \pm \varphi_{0}\right)\right| \operatorname{sign} z_{0}, \\
m_{0}^{\prime}=-\kappa \sin \left(\theta_{0} \pm \varphi_{0}\right) \operatorname{sign} \cos \left(\theta_{0} \pm \varphi_{0}\right) \operatorname{sign} z_{0}+\mathrm{i} \frac{\beta \kappa^{3} \operatorname{sign} z_{0}}{\left|\cos \left(\theta_{0} \pm \varphi_{0}\right)\right|}
\end{gathered}
$$

This result corresponds to a source contained inside the strip $\left|z_{0}\right|<b$, such that $f_{0}\left(x_{0}, z_{0}\right)=0$ for $\left|z_{0}\right|>b$, and is valid outside this strip for $\left|z_{0}\right|>b$.

\subsection{Source with horizontal and vertical axes}

The relation between the different expressions of the waves is tedious to investigate in the general case, owing to the number of possibilities to consider depending on the value of $\varphi_{0}$ compared with $\pm \theta_{0}$ and $\pm\left(\pi / 2-\theta_{0}\right)$. With future application to $\S 8.1$ in mind, we consider the particular case of a source of horizontal semi-axis $a$ and vertical semi-axis $b$, and focus on expressions (4.26) and (6.2). For the former, we set $\varphi_{0}=0$. The lengths $c_{+}$and $c_{-}$merge into a single $c$, and $d_{+}$and $d_{-}$into a single $d$, with

$$
c=\left(a^{2} \cos ^{2} \theta_{0}+b^{2} \sin ^{2} \theta_{0}\right)^{1 / 2}, \quad d=\frac{\left(a^{4} \cos ^{2} \theta_{0}+b^{4} \sin ^{2} \theta_{0}\right)^{1 / 2}}{c} .
$$

Similarly, $\Theta_{+}$and $\Theta_{-}$merge into $\Theta_{0}$, and $\chi_{+}$and $\chi_{-}$into $\chi_{0}$, with

$$
\Theta_{0}=\arctan \left(\frac{b}{a} \tan \theta_{0}\right), \quad \chi_{0}=\arctan \left(\frac{b^{2}}{a^{2}} \tan \theta_{0}\right) .
$$


Expression (4.26) becomes, for $\left|\zeta_{+}\right|>a b / d$ and $\left|\zeta_{-}\right|>a b / d$,

$$
\begin{aligned}
\boldsymbol{u}=\frac{\exp \left(-\mathrm{i} \omega_{0} t\right)}{4 \pi} \sum_{ \pm} \boldsymbol{e}_{z_{ \pm}} \operatorname{sign} \zeta_{ \pm} \int_{0}^{\infty} F_{ \pm} & \left(K_{ \pm}= \pm \kappa c \operatorname{sign} \zeta_{ \pm}, M_{ \pm}=\mathrm{i} \beta \kappa^{3} \frac{a b}{c} \operatorname{sign} \zeta_{ \pm}\right) \\
& \times \exp \left(-\beta \kappa^{3} \frac{d}{c}\left|\zeta_{ \pm}\right|\right) \exp \left( \pm \mathrm{i} \kappa x_{ \pm} \operatorname{sign} \zeta_{ \pm}\right) \mathrm{d} \kappa
\end{aligned}
$$

where

$$
\frac{d}{c} \zeta_{ \pm}= \pm x \frac{b^{2}}{c^{2}} \sin \theta_{0}+z \frac{a^{2}}{c^{2}} \cos \theta_{0}
$$

For (6.2), we set first $\varphi_{0}=\pi / 2$ to obtain, for $|x|>a$,

$$
\begin{aligned}
& \boldsymbol{u}=\frac{\exp \left(-\mathrm{i} \omega_{0} t\right)}{4 \pi} \operatorname{sign} x \sum_{ \pm}( \pm) \boldsymbol{e}_{z_{ \pm}} \int_{0}^{\infty} \exp \left(-\beta \kappa^{3}|x| / \sin \theta_{0}\right) \exp \left(\mathrm{i} \kappa x_{ \pm} \operatorname{sign} x\right) \\
& \quad \times f\left[k=\kappa \cos \theta_{0} \operatorname{sign} x+\mathrm{i} \beta \kappa^{3} \operatorname{sign} x / \sin \theta_{0}, m=\mp \kappa \sin \theta_{0} \operatorname{sign} x\right] \mathrm{d} \kappa
\end{aligned}
$$

and then $\varphi_{0}=0$ to obtain, for $|z|>b$,

$$
\begin{aligned}
\boldsymbol{u}=\frac{\exp \left(-\mathrm{i} \omega_{0} t\right)}{4 \pi} & \operatorname{sign} z \sum_{ \pm} \boldsymbol{e}_{z_{ \pm}} \int_{0}^{\infty} \exp \left(-\beta \kappa^{3}|z| / \cos \theta_{0}\right) \exp \left( \pm \mathrm{i} \kappa x_{ \pm} \operatorname{sign} z\right) \\
& \times f\left[k= \pm \kappa \cos \theta_{0} \operatorname{sign} z, m=-\kappa \sin \theta_{0} \operatorname{sign} z+\mathrm{i} \beta \kappa^{3} \operatorname{sign} z / \cos \theta_{0}\right] \mathrm{d} \kappa
\end{aligned}
$$

The relations between these expressions follow from the low-viscosity assumption $\beta / c^{2} \ll 1$. Consider (6.6), where the condition $\left|K_{ \pm}\right|=\kappa c=O(1)$ implies $\beta \kappa^{2} \ll 1$. In the sectors $b^{2}|x| \sin \theta_{0}>a^{2}|z| \cos \theta_{0}$, where sign $\zeta_{ \pm}= \pm \operatorname{sign} x$, the change of variable

$$
\kappa^{\prime}=\kappa-\mathrm{i} \beta \kappa^{3} \frac{a^{2}}{c^{2}} \cot \theta_{0}
$$

maps, to leading order, the path of integration onto itself and reduces (6.6) to (6.8). In the sectors $b^{2}|x| \sin \theta_{0}<a^{2}|z| \cos \theta_{0}$, where $\operatorname{sign} \zeta_{ \pm}=\operatorname{sign} z$, the change of variable

$$
\kappa^{\prime}=\kappa+\mathrm{i} \beta \kappa^{3} \frac{b^{2}}{c^{2}} \tan \theta_{0}
$$

reduces (6.6) to (6.9). Such changes of variable were first introduced by Kistovich \& Chashechkin $(1994,1995)$.

\subsection{Relevance}

Several equivalent expressions of the waves have been obtained, showing that the incompatibility highlighted in figure 3 was only apparent. At each location two wave beams are received, corresponding to two half-arms of the wavenumber curve. The separation line between the beams originating from the two halves of each arm, and the attenuation of the waves at each wavenumber in proportion to the distance to this line, are all artifacts of the way the waves are calculated. When the beams are properly superposed, the three approaches illustrated in figures 3(a), (b) and (c), corresponding to (6.1), (6.9) and (6.6), respectively, yield identical results in their common areas of validity. Each expression is better suited to a particular type of source: (6.1) to a circular source, (4.26) and (6.6) to an elliptic source, (6.2), (6.8) and (6.9) to a source contained in a strip, and (5.9), (5.15) and (5.19) to a line source. Before applying these expressions to specific sources and comparing them with experiment, we briefly consider unsteady effects. 


\section{Unsteady effects}

In laboratory experiments, depending on the setup, it may not be possible to wait long enough after the source of the waves has started to operate, for a steady state to be reached. Unsteady effects are observed, which must be taken into account when comparing with the theory (Ermanyuk \& Gavrilov 2005; Voisin, Ermanyuk \& Flór 2011). A simple way of achieving this aim is to consider impulsive start-up, namely to write $q=f(x, z) H(t) \exp \left(-\mathrm{i} \omega_{0} t\right)$, with $H(t)=0$ for $t<0$ and 1 for $t>0$ the Heaviside step function, and investigate the large-time limit $N t \gg 1$. This procedure has been outlined by Lighthill (1960).

Neglecting viscosity and using the transform, taken from table 5 of Voisin (2003),

$$
\int_{0}^{\infty} \mathrm{e}^{\mathrm{i} \omega t} \mathrm{~d} t=\frac{\mathrm{i}}{\omega+\mathrm{i} 0}=\lim _{\epsilon \rightarrow 0_{+}} \frac{\mathrm{i}}{\omega+\mathrm{i} \epsilon},
$$

we obtain

$$
\boldsymbol{u}=\frac{1}{8 \pi^{3}} \lim _{\epsilon \rightarrow 0_{+}} \iiint \frac{\omega^{2} \boldsymbol{k}-N^{2} k \boldsymbol{e}_{x}}{\omega^{2} \kappa^{2}-N^{2} k^{2}} \frac{f(k, m)}{\omega-\omega_{0}+\mathrm{i} \epsilon} \exp [\mathrm{i}(k x+m z-\omega t)] \mathrm{d} k \mathrm{~d} m \mathrm{~d} \omega .
$$

For large times $N t \gg 1$, the dominant asymptotic contribution to this inverse transform arises from an $O(1 / t)$ vicinity of the singular frequency $\omega=\omega_{0}-\mathrm{i} \epsilon$, yielding

$$
\begin{aligned}
\boldsymbol{u} \sim \frac{1}{8 \pi^{3}} \lim _{\epsilon \rightarrow 0_{+}} \int \mathrm{d} \omega & \frac{\exp (-\mathrm{i} \omega t)}{\omega} \iint \mathrm{d} k \mathrm{~d} m f(k, m) \exp [\mathrm{i}(k x+m z)] \\
& \times \frac{m \boldsymbol{e}_{z} \cos ^{2} \theta_{0}-k \boldsymbol{e}_{x} \sin ^{2} \theta_{0}+2\left[\left(\omega-\omega_{0}+\mathrm{i} \epsilon\right) / N\right] \boldsymbol{k} \cos \theta_{0}}{m^{2} \cos ^{2} \theta_{0}-k^{2} \sin ^{2} \theta_{0}+2\left[\left(\omega-\omega_{0}+\mathrm{i} \epsilon\right) / N\right] \kappa^{2} \cos \theta_{0}},
\end{aligned}
$$

where $\left|\omega-\omega_{0}+\mathrm{i} \epsilon\right| / N=O[1 /(N t)] \ll 1$. The resulting dispersion relation,

$$
B(\omega+\mathrm{i} \epsilon, \boldsymbol{k}) \sim N^{2}\left[m_{ \pm} m_{\mp}+2 \frac{\omega-\omega_{0}+\mathrm{i} \epsilon}{N}\left(k_{ \pm}^{2}+m_{ \pm}^{2}\right) \cos \theta_{0}\right]=0,
$$

is of the same form as that (2.13) including Lighthill's radiation condition. Proceeding as in $\S 4.1$, we obtain the poles

$$
M_{ \pm} \sim \pm \frac{a b}{c_{ \pm}^{2}} \frac{\omega-\omega_{0}+\mathrm{i} \epsilon}{N \sin \theta_{0}} K_{ \pm}
$$

of which the appropriate deformation of contour selects

$$
M_{ \pm} \sim \frac{a b}{c_{ \pm}^{2}} \frac{\omega-\omega_{0}+\mathrm{i} \epsilon}{N \sin \theta_{0}}\left|K_{ \pm}\right| \operatorname{sign} Z_{ \pm} \quad \text { with } \quad \operatorname{sign} K_{ \pm}= \pm \operatorname{sign} Z_{ \pm} .
$$

Applying the residue theorem to integration over $M_{ \pm}$, and the inverse transform

$$
\lim _{\epsilon \rightarrow 0_{+}} \int \frac{\mathrm{e}^{-\mathrm{i} \omega t}}{\omega+\mathrm{i} \epsilon} \mathrm{d} \omega=\int \frac{\mathrm{e}^{-\mathrm{i} \omega t}}{\omega+\mathrm{i} 0} \mathrm{~d} \omega=2 \pi H(t)
$$

to integration over $\omega$, the result only differs from (4.16) by the inclusion of a factor

$$
H\left(t-\frac{a b}{c_{ \pm}^{2}} \frac{\left|K_{ \pm} Z_{ \pm}\right|}{N \sin \theta_{0}}\right)=H\left(1-\alpha \kappa \frac{d_{ \pm}}{c_{ \pm}}\left|\zeta_{ \pm}\right|\right)
$$

inside the integrand, with

$$
\alpha=\frac{1}{N t \sin \theta_{0}}
$$


To leading order, viscosity and unsteadiness are seen to induce independent low-pass cutoff factors, respectively exponential and step-like, in the integral expression of the waves. Accordingly, their effects can be simply superposed yielding, for $\left|\zeta_{+}\right|>a b / d_{+}$ and $\left|\zeta_{-}\right|>a b / d_{-}$,

$$
\begin{aligned}
\boldsymbol{u}=\frac{\exp \left(-\mathrm{i} \omega_{0} t\right)}{4 \pi} & \sum_{ \pm} \boldsymbol{e}_{z_{ \pm}} \operatorname{sign} \zeta_{ \pm} \int_{0}^{\infty} F_{ \pm}\left(K_{ \pm}= \pm \kappa c_{ \pm} \operatorname{sign} \zeta_{ \pm}, M_{ \pm}=\mathrm{i} \beta \kappa^{3} \frac{a b}{c_{ \pm}} \operatorname{sign} \zeta_{ \pm}\right) \\
& \times H\left(1-\alpha \kappa \frac{d_{ \pm}}{c_{ \pm}}\left|\zeta_{ \pm}\right|\right) \exp \left(-\beta \kappa^{3} \frac{d_{ \pm}}{c_{ \pm}}\left|\zeta_{ \pm}\right|\right) \exp \left( \pm \mathrm{i} \kappa x_{ \pm} \operatorname{sign} \zeta_{ \pm}\right) \mathrm{d} \kappa
\end{aligned}
$$

The alternative expressions of the waves for bluff forcing in $\S 6$ and the expressions for line forcing in $\$ 5$ can easily be modified in the same way.

\section{Applications}

The analysis of the preceding sections is now applied to four oscillating bodies: an elliptic cylinder, a vertical barrier, a wave generator and a thin topography. Experimental measurements are available for all of them, to which the theory can be compared. All measurements were made once a steady state had been reached, removing the need for the unsteady correction of $\S 7$. On the assumption of large Stokes number, the viscous boundary layer around the body is negligible and the boundary condition approximately free-slip. The first body is of the bluff type considered in $\$ \S 4$ and 6 , and the next three of the line type considered in $\S 5$.

\subsection{Elliptic cylinder}

For a long time, following the pioneering experiments of Mowbray \& Rarity (1967), the preferred way of generating monochromatic internal waves in the laboratory has been the oscillations of a horizontal circular cylinder. Hurley (1997) and Hurley \& Keady (1997) calculated the waves analytically, and Sutherland et al. $(1999,2000)$ and Zhang et al. (2007) compared their predictions with experiment, the latter paying particular attention to the near field. Winters \& Armi (2013) investigated the flow numerically. For this particular geometry, the present theory and the Hurley-Keady theory yield identical results.

The same is not true for the elliptic cylinder. The predictions of Hurley (1997) and Hurley \& Keady (1997) were compared with experiment by Sutherland \& Linden (2002), for the case when the elliptic cross section has horizontal and vertical axes. To apply the present theory to this configuration, a representation of the cylinder as a source of mass is required. It is obtained by combining the boundary integral method with the method, based on coordinate stretching and analytic continuation, introduced by Bryan (1889) for inertial waves and Hurley (1972) for internal waves. The derivation will be reported elsewhere; a summary may be found in Voisin (2009) for a sphere.

An elliptic cylinder of horizontal semi-axis $a$ and vertical semi-axis $b$, oscillating at the velocity $(U, W) \mathrm{e}^{-\mathrm{i} \omega_{0} t}$, has the representation

$$
f(x, z)=\left[\left(1+\mathrm{i} \frac{b}{a} \tan \theta_{0}\right) U \frac{x}{a^{2}}+\left(1-\mathrm{i} \frac{a}{b} \cot \theta_{0}\right) W \frac{z}{b^{2}}\right] \delta\left[\left(\frac{x^{2}}{a^{2}}+\frac{z^{2}}{b^{2}}\right)^{1 / 2}-1\right],
$$


of spectrum

$$
f(k, m)=-2 \mathrm{i} \pi a b\left[\left(1+\mathrm{i} \frac{b}{a} \tan \theta_{0}\right) U k+\left(1-\mathrm{i} \frac{a}{b} \cot \theta_{0}\right) W m\right] \frac{\mathrm{J}_{1}\left[\left(k^{2} a^{2}+m^{2} b^{2}\right)^{1 / 2}\right]}{\left(k^{2} a^{2}+m^{2} b^{2}\right)^{1 / 2}},
$$

with $\mathrm{J}_{1}$ a Bessel function. At this stage two remarks must be made. First, the spectrum may only be derived from known integrals for real wavenumbers $k$ and $m$, and the result has been continued analytically to complex wavenumbers. Secondly, the source being a distribution of order 0 rather than a proper function, the analysis of $\S \S 4$ and 6 has been extended as discussed in Appendix A, with identical result.

We introduce, as did Hurley (1997), the notation

$$
\alpha_{ \pm}=\frac{\mathrm{e}^{\mathrm{i} \Theta_{0}}}{2}\left(\frac{a}{c} W \mp \mathrm{i} \frac{b}{c} U\right)=\frac{\left(a \cos \theta_{0}+\mathrm{i} b \sin \theta_{0}\right)(a W \mp \mathrm{i} b U)}{2 c^{2}},
$$

such that

$$
f_{ \pm}\left(k_{ \pm}, m_{ \pm}=0\right)= \pm 4 \pi c \alpha_{ \pm} \mathrm{J}_{1}\left(k_{ \pm} c\right) \text {. }
$$

When using (6.6), the viscous correction to the wavenumber inside the source spectrum is of the second order in the small parameter $\beta / c^{2}$, since $k^{2} a^{2}+m^{2} b^{2}=K_{ \pm}^{2}+M_{ \pm}^{2}$ with $\left|K_{ \pm}\right|=\kappa c=O(1)$ and $\left|M_{ \pm}\right|=O\left(\beta \kappa^{2}\right)=O\left(\beta / c^{2}\right)$. It is thus negligible. We obtain, for $\left|\zeta_{+}\right|>a b / d$ and $\left|\zeta_{-}\right|>a b / d$,

$$
\boldsymbol{u}=c \exp \left(-\mathrm{i} \omega_{0} t\right) \sum_{ \pm} \alpha_{ \pm} \boldsymbol{e}_{z_{ \pm}} \int_{0}^{\infty} \mathrm{J}_{1}(\kappa c) \exp \left(-\beta \kappa^{3} \frac{d}{c}\left|\zeta_{ \pm}\right|\right) \exp \left( \pm \mathrm{i} \kappa x_{ \pm} \operatorname{sign} \zeta_{ \pm}\right) \mathrm{d} \kappa
$$

By contrast, when using the other expressions, the viscous correction is of the first order inside the source spectrum, hence significant. We obtain from (6.1), for $\left|z_{+}\right|>$ $\left(a^{2} \sin ^{2} \theta_{0}+b^{2} \cos ^{2} \theta_{0}\right)^{1 / 2}$ and $\left|z_{-}\right|>\left(a^{2} \sin ^{2} \theta_{0}+b^{2} \cos ^{2} \theta_{0}\right)^{1 / 2}$,

$$
\begin{aligned}
\boldsymbol{u}=c \exp \left(-\mathrm{i} \omega_{0} t\right) \sum_{ \pm} \alpha_{ \pm} \boldsymbol{e}_{z_{ \pm}} \int_{0}^{\infty} \mathrm{J}_{1}(\kappa c+ & \left.\mathrm{i} \beta \kappa^{3} \frac{a^{2}-b^{2}}{c} \sin \theta_{0} \cos \theta_{0}\right) \\
& \times \exp \left(-\beta \kappa^{3}\left|z_{ \pm}\right|\right) \exp \left( \pm \mathrm{i} \kappa x_{ \pm} \operatorname{sign} z_{ \pm}\right) \mathrm{d} \kappa,
\end{aligned}
$$

and similarly from (6.8), for $|x|>a$,

$$
\begin{aligned}
\boldsymbol{u}= & \exp \left[-\mathrm{i}\left(\omega_{0} t-\Theta_{0}\right)\right] \int_{0}^{\infty} \mathrm{J}_{1}\left(\kappa c+\mathrm{i} \beta \kappa^{3} \frac{a^{2}}{c} \cot \theta_{0}\right) \exp \left(-\beta \kappa^{3}|x| / \sin \theta_{0}\right) \\
& \times \exp \left(\mathrm{i} \kappa|x| \cos \theta_{0}\right)\left\{\boldsymbol{e}_{z} \cos \theta_{0}\left[a W \cos \left(\kappa z \sin \theta_{0}\right)-b U \sin \left(\kappa z \sin \theta_{0}\right) \operatorname{sign} x\right]\right. \\
& \left.-\mathrm{i} \boldsymbol{e}_{x} \sin \theta_{0}\left[a W \sin \left(\kappa z \sin \theta_{0}\right) \operatorname{sign} x+b U \cos \left(\kappa z \sin \theta_{0}\right)\right]\right\} \mathrm{d} \kappa,
\end{aligned}
$$

and from (6.9), for $|z|>b$,

$$
\begin{aligned}
\boldsymbol{u}= & \exp \left[-\mathrm{i}\left(\omega_{0} t-\Theta_{0}\right)\right] \int_{0}^{\infty} \mathrm{J}_{1}\left(\kappa c-\mathrm{i} \beta \kappa^{3} \frac{b^{2}}{c} \tan \theta_{0}\right) \exp \left(-\beta \kappa^{3}|z| / \cos \theta_{0}\right) \\
& \times \exp \left(-\mathrm{i} \kappa|z| \sin \theta_{0}\right)\left\{\boldsymbol{e}_{z} \cos \theta_{0}\left[a W \cos \left(\kappa x \cos \theta_{0}\right)+b U \sin \left(\kappa x \cos \theta_{0}\right) \operatorname{sign} z\right]\right. \\
& \left.+\mathrm{i} \boldsymbol{e}_{x} \sin \theta_{0}\left[a W \sin \left(\kappa z \cos \theta_{0}\right) \operatorname{sign} z-b U \cos \left(\kappa z \cos \theta_{0}\right)\right]\right\} \mathrm{d} \kappa .
\end{aligned}
$$

The classical theory of Hurley \& Keady (1997) corresponds to applying (8.6) everywhere while omitting the viscous correction inside the Bessel function.

Now, as discussed in $\S 2.1$, the above domains of applicability are those for which the validity of the results has been ascertained for a generic elliptic source, based on bounds such as (2.20) and (4.13). If we consider instead the convergence of the above integrals 
for the elliptic cylinder, larger domains are obtained, starting from the lines where the coordinates involved in beam separation and viscous attenuation take their values at the critical points where the critical wave rays are tangential to the cylinder. Specifically, (8.5) converges everywhere, (8.6) for

$$
\left|z_{+}\right|>\frac{\left|a^{2}-b^{2}\right|}{c} \sin \theta_{0} \cos \theta_{0} \text { and }\left|z_{-}\right|>\frac{\left|a^{2}-b^{2}\right|}{c} \sin \theta_{0} \cos \theta_{0},
$$

(8.7) for

$$
|x|>\frac{a^{2}}{c} \cos \theta_{0}
$$

and (8.8) for

$$
|z|>\frac{b^{2}}{c} \sin \theta_{0}
$$

These domains remain relevant even in the absence of viscosity, if the infinitesimal imaginary part $\epsilon$ added to the frequency by the radiation condition is kept during the whole calculation and the limit $\epsilon \rightarrow 0$ applied only at the very end.

In the inviscid case, the Fourier transform

$$
\int_{0}^{\infty} \mathrm{J}_{1}(k) \exp (\mathrm{i} k x) \mathrm{d} k=1-\frac{x}{\left[(x+\mathrm{i} 0)^{2}-1\right]^{1 / 2}},
$$

taken from table 5 of Voisin (2003), turns (8.5) into

$$
\boldsymbol{u}=\exp \left(-\mathrm{i} \omega_{0} t\right) \sum_{ \pm} \alpha_{ \pm} \boldsymbol{e}_{z_{ \pm}}\left\{1-\frac{x_{ \pm}}{\left[\left(x_{ \pm} \pm \mathrm{i} 0 \operatorname{sign} \zeta_{ \pm}\right)^{2}-c^{2}\right]^{1 / 2}}\right\},
$$

and similarly for (8.6), (8.7) and (8.8) with $\operatorname{sign} \zeta_{ \pm}$replaced by sign $z_{ \pm}$, sign $x$ and $\operatorname{sign} z$, respectively. Solution (8.13) is identical to that (3.28)-(3.29) from Hurley (1997). At the cylinder, we introduce the excentric angle $\eta$ such that

$$
x=a \cos \eta, \quad z=b \sin \eta
$$

to obtain

$$
x_{ \pm}=c \cos \left(\eta \pm \Theta_{0}\right), \quad \zeta_{ \pm}=\frac{a b}{d} \sin \left(\eta \pm \Theta_{0}\right) .
$$

The solution becomes

$$
\boldsymbol{u}=\exp \left(-\mathrm{i} \omega_{0} t\right) \sum_{ \pm} \alpha_{ \pm} \boldsymbol{e}_{z_{ \pm}}\left[1 \pm \mathrm{i} \cot \left(\eta \pm \Theta_{0}\right)\right]
$$

and satisfies the free-slip boundary condition at the cylinder,

$$
\boldsymbol{n} \cdot \boldsymbol{u}=\boldsymbol{n} \cdot\left(U \boldsymbol{e}_{x}+W \boldsymbol{e}_{z}\right) \exp \left(-i \omega_{0} t\right)
$$

where the outward normal $\boldsymbol{n}$ is given by

$$
\boldsymbol{n}=\frac{b \boldsymbol{e}_{x} \cos \eta+a \boldsymbol{e}_{z} \sin \eta}{\left(a^{2} \sin ^{2} \eta+b^{2} \cos ^{2} \eta\right)^{1 / 2}} .
$$

Accordingly, (8.13) applies everywhere in the fluid. For the other solutions, we write

$$
\begin{aligned}
\frac{x}{\left[(x \pm \mathrm{i} 0)^{2}-1\right]^{1 / 2}} & =\frac{|x|}{\left|x^{2}-1\right|^{1 / 2}} \quad(|x|>1), \\
& =\mp \mathrm{i} \frac{x}{\left|1-x^{2}\right|^{1 / 2}} \quad(|x|<1) .
\end{aligned}
$$


All four solutions are identical outside the wave beams, for $\left|x_{ \pm}\right|>c$. Inside the beams, for $\left|x_{ \pm}\right|<c$, the solutions (8.6), (8.7) and (8.8) are valid wherever $z_{ \pm}, x$ and $z$ have the same sign as $\zeta_{ \pm}$, that is for (8.9), (8.10) and (8.11), respectively.

Consistent with (8.19b), the velocity is discontinuous across the portion $\left|x_{ \pm}\right|<c$ of the beam separation lines and singular at their extremities $\left|x_{ \pm}\right|=c$, where the lines intersect the critical rays. The discontinuity persists in the presence of viscosity, since the effect of viscosity vanishes at the separation lines. When (8.5) is used, the separation lines are $\zeta_{ \pm}=0$ and the discontinuity takes place in between the critical points $\left[|x|=\left(a^{2} / c\right) \cos \theta_{0},|z|=\left(b^{2} / c\right) \sin \theta_{0}\right]$, that is inside the cylinder. When (8.6) is used, the separation lines are $z_{ \pm}=0$ and the discontinuity penetrates into the fluid, up to the points $\left(|x|=c \cos \theta_{0},|z|=c \sin \theta_{0}\right)$. When (8.7) is used, the discontinuity extends along the line $x=0$ up to $|z|=c / \sin \theta_{0}$ inside the fluid, and similarly when (8.8) is used it extends along the line $z=0$ up to $|x|=c / \cos \theta_{0}$. Once the domains of validity (8.9)-(8.11) are taken into account, the discontinuities become irrelevant.

Sutherland \& Linden (2002) considered the vertical oscillations of two elliptic cylinders: one, with horizontal semi-axis $a=2.10 \mathrm{~cm}$, vertical semi-axis $b=1.12 \mathrm{~cm}$ and aspect ratio $a / b \approx 2$; the other, with horizontal semi-axis $a=2.52 \mathrm{~cm}$, vertical semi-axis $b=0.86 \mathrm{~cm}$ and aspect ratio $a / b \approx 3$. These dimensions were chosen so as to keep the average radius $(a+b) / 2$ approximately the same, close to $1.67 \mathrm{~cm}$. Synthetic schlieren was used to measure the time derivative of the buoyancy frequency disturbance, $N_{t}^{2}=$ $-N^{2} \partial w / \partial z$. The outcome was compared with the predictions of Hurley \& Keady (1997). The buoyancy frequency was $N=0.97 \mathrm{~s}^{-1}$. The kinematic viscosity was not specified and has been taken as $\nu=1 \mathrm{~mm}^{2} \mathrm{~s}^{-1}$.

Choosing, as did Sutherland \& Linden (2002), the phase of the oscillation $\phi=\omega_{0} t$ to be zero at the instant when the cylinder moves downwards through the midpoint of its oscillation, and introducing the (real positive) oscillation amplitude $A$, we write the position of the cylinder as $\operatorname{Re}\left[-\mathrm{i} A \boldsymbol{e}_{z} \exp \left(-\mathrm{i} \omega_{0} t\right)\right]$, so that $W=-\omega_{0} A$. Normalizing with

$$
A_{N_{t}^{2}}=N^{3} \frac{a A}{2 c^{2}} \sin \theta_{0} \cos ^{2} \theta_{0},
$$

we obtain

$$
\begin{aligned}
\frac{N_{t}^{2}}{A_{N_{t}^{2}}}=-\mathrm{i} c^{2} \exp \left[-\mathrm{i}\left(\omega_{0} t\right.\right. & \left.\left.-\Theta_{0}\right)\right] \int_{0}^{\infty} \kappa \mathrm{J}_{1}(\kappa c) \\
& \times \sum_{ \pm} \operatorname{sign} \zeta_{ \pm} \exp \left(-\beta \kappa^{3} \frac{d}{c}\left|\zeta_{ \pm}\right|\right) \exp \left( \pm \mathrm{i} \kappa x_{ \pm} \operatorname{sign} \zeta_{ \pm}\right) \mathrm{d} \kappa
\end{aligned}
$$

valid everywhere;

$$
\begin{aligned}
\frac{N_{t}^{2}}{A_{N_{t}^{2}}}=-\mathrm{i} c^{2} \exp \left[-\mathrm{i}\left(\omega_{0} t-\right.\right. & \left.\left.\Theta_{0}\right)\right] \int_{0}^{\infty} \kappa \mathrm{J}_{1}\left(\kappa c+\mathrm{i} \beta \kappa^{3} \frac{a^{2}-b^{2}}{c} \sin \theta_{0} \cos \theta_{0}\right) \\
& \times \sum_{ \pm} \operatorname{sign} z_{ \pm} \exp \left(-\beta \kappa^{3}\left|z_{ \pm}\right|\right) \exp \left( \pm \mathrm{i} \kappa x_{ \pm} \operatorname{sign} z_{ \pm}\right) \mathrm{d} \kappa,
\end{aligned}
$$

valid for $\left|z_{+}\right|>\left(\left|a^{2}-b^{2}\right| / c\right) \sin \theta_{0} \cos \theta_{0}$ and $\left|z_{-}\right|>\left(\left|a^{2}-b^{2}\right| / c\right) \sin \theta_{0} \cos \theta_{0}$;

$$
\begin{aligned}
\frac{N_{t}^{2}}{A_{N_{t}^{2}}}=-2 c^{2} \exp \left[-\mathrm{i}\left(\omega_{0} t-\Theta_{0}\right)\right] \int_{0}^{\infty} \kappa \mathrm{J}_{1}\left(\kappa c+\mathrm{i} \beta \kappa^{3} \frac{a^{2}}{c} \cot \theta_{0}\right) \\
\times \exp \left(-\beta \kappa^{3}|x| / \sin \theta_{0}\right) \exp \left(\mathrm{i} \kappa|x| \cos \theta_{0}\right) \sin \left(\kappa z \sin \theta_{0}\right) \mathrm{d} \kappa
\end{aligned}
$$


valid for $|x|>\left(a^{2} / c\right) \cos \theta_{0}$; and

$$
\begin{aligned}
\frac{N_{t}^{2}}{A_{N_{t}^{2}}}=-2 \mathrm{i} c^{2} & \exp \left[-\mathrm{i}\left(\omega_{0} t-\Theta_{0}\right)\right] \int_{0}^{\infty} \kappa \mathrm{J}_{1}\left(\kappa c-\mathrm{i} \beta \kappa^{3} \frac{b^{2}}{c} \tan \theta_{0}\right) \\
& \times \operatorname{sign} z \exp \left(-\beta \kappa^{3}|z| / \cos \theta_{0}\right) \cos \left(\kappa x \cos \theta_{0}\right) \exp \left(-\mathrm{i} \kappa|z| \sin \theta_{0}\right) \mathrm{d} \kappa
\end{aligned}
$$

valid for $|z|>\left(b^{2} / c\right) \sin \theta_{0}$. The classical theory of Hurley \& Keady (1997) gives

$$
\begin{aligned}
\left(\frac{N_{t}^{2}}{A_{N_{t}^{2}}}\right)_{\mathrm{c}}=-\mathrm{i} c^{2} \exp \left[-\mathrm{i}\left(\omega_{0} t\right.\right. & \left.\left.-\Theta_{0}\right)\right] \int_{0}^{\infty} \kappa \mathrm{J}_{1}(\kappa c) \\
& \times \sum_{ \pm} \operatorname{sign} z_{ \pm} \exp \left(-\beta \kappa^{3}\left|z_{ \pm}\right|\right) \exp \left( \pm \mathrm{i} \kappa x_{ \pm} \operatorname{sign} z_{ \pm}\right) \mathrm{d} \kappa
\end{aligned}
$$

The relevant non-dimensional parameters are the Stokes number $S t=\omega_{0}(a+b)^{2} /(4 v)$ and the Keulegan-Carpenter number $\mathrm{Ke}=2 A /(a+b)$.

We focus on figures 8,9 and 10 of Sutherland \& Linden (2002), corresponding to the range of parameters for which no second harmonic wave is generated and the waves are close to linear. The waves were measured for $A=0.32 \mathrm{~cm}$ in the first quadrant, where the dominant beam propagates upward to the right. The along-beam profiles at the beam axis $x_{+}=0$ in their figure 9 and the across-beam profile at distance $z_{+}=20 \mathrm{~cm}$ in their figure 10 essentially belong to the far field, namely to distances from the cylinder larger than one to three times its average radius say; there, the preceding expressions all coincide with one another and with the Hurley-Keady theory. We switch instead to the contour maps in their figure 8. Application of the present 'best' expression (8.21) is shown in figure 5. In the far field, where (8.21) and the Hurley-Keady theory (8.25) yield identical results, the agreement with experiment is good.

The differences between the theories appear in the near field, shown in figure 6 . To better illustrate the underlying structure, the waves have been plotted both inside and outside the cylinder. The present theory (8.21) predicts singularities at the critical points, visible as maxima in Sutherland's \& Linden's (2002) figure 8, especially subfigures ( $c, d)$. By contrast, the Hurley-Keady theory (8.25) puts the singularities closer to the vertical, inside the fluid and connected to the cylinder by segments of discontinuity.

The relation between all four solutions (8.21)-(8.24) is illustrated in figures 7 and 8 for the largest and smallest angles of propagation to the vertical, corresponding to subfigures $(a)$ and $(d)$ of Sutherland \& Linden (2002), respectively. When viscosity is not taken into account in the source spectrum, the critical points are put inside the fluid by the last three solutions, connected to the cylinder by segments of discontinuity, and the solutions exhibit significant differences with one another. When viscosity is taken into account, all four solutions predict the correct positions of the critical points and are in close agreement with one another in their common domains of validity.

The remaining small discrepancies are caused by the finite value of $S t$. Consistent with $\S 6.2$, when the waves propagate closer to the horizontal in figure 7 , the region $b^{2}|x| \sin \theta_{0}>a^{2}|z| \cos \theta_{0}$ is more extended and the solution (8.23) provides the best approximation to (8.21). When the waves propagate closer to the vertical in figure 8 , the region $b^{2}|x| \sin \theta_{0}<a^{2}|z| \cos \theta_{0}$ is more extended and (8.24) provides the best approximation. Accordingly, (8.23) and (8.24) offer simpler alternatives to (8.21), relevant at low and high frequencies respectively. The solution (8.22) does not seem to offer any particular advantage over (8.21). 

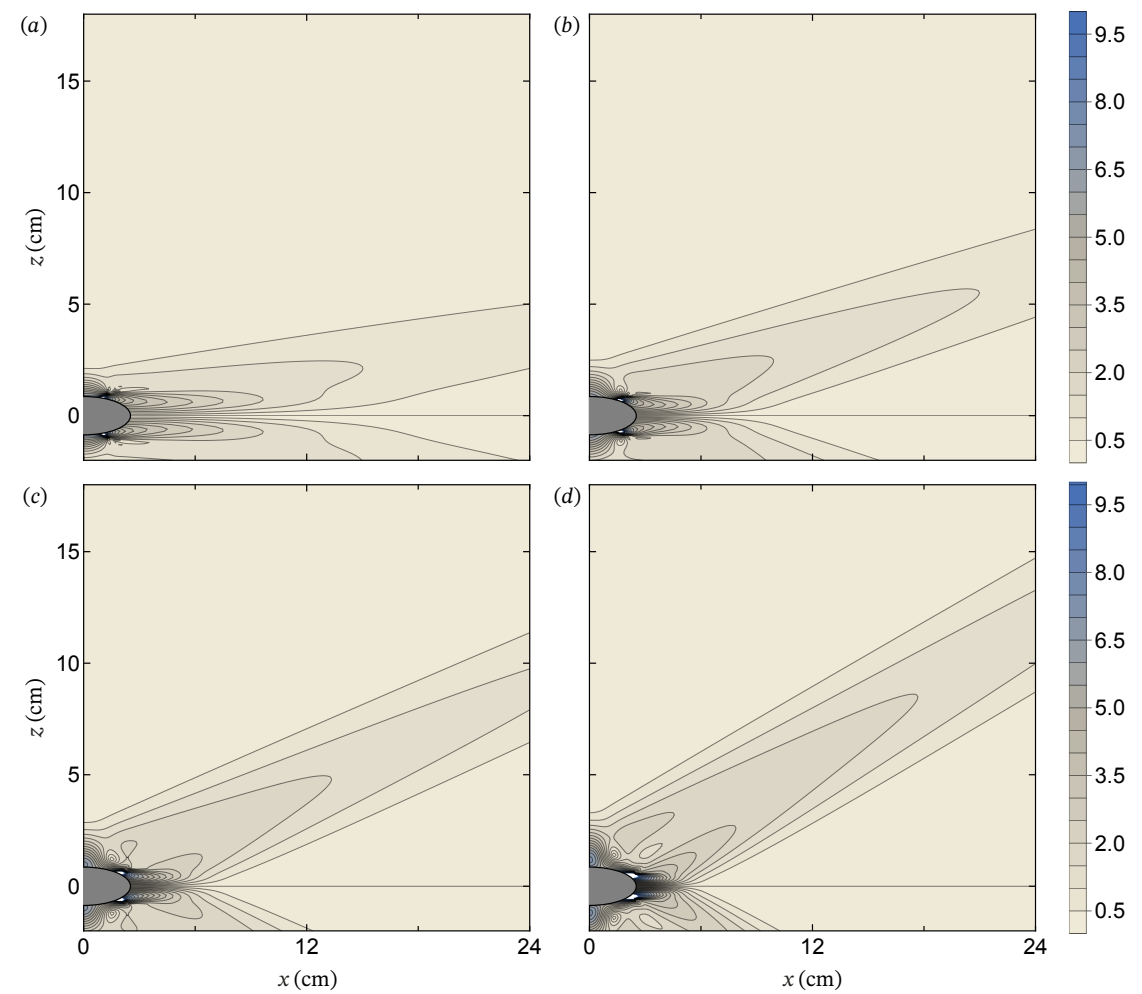

FIGURE 5. Contour maps of $\left|N_{t}^{2}\right| / A_{N_{t}^{2}}$, as predicted by (8.21), for the oscillations at relative frequencies (a) $\omega_{0} / N=0.15,(b) \omega_{0} / N=0.26,(c) \omega_{0} / N=0.35$ and $(d) \omega_{0} / N=0.44$ of the elliptic cylinder of aspect ratio $a / b \approx 3$ in figure 8 of Sutherland \& Linden (2002). The waves propagate at the angles $(a) \theta_{0}=81^{\circ}$, (b) $\theta_{0}=75^{\circ}$, (c) $\theta_{0}=70^{\circ}$ and $(d) \theta_{0}=64^{\circ}$ to the vertical, with Stokes numbers $(a) S t=42$, (b) $S t=72$, (c) $S t=97$ and (d) $S t=120$, and Keulegan-Carpenter number $K e=0.19$. The white areas correspond to off-scale values of the plotted quantity.

\subsection{Vertical barrier}

The first study of monochromatic internal waves in the laboratory, by Görtler (1943), used the horizontal oscillations of a vertical plate. The plate was thick, piercing through the surface of the fluid down a depth $b=6 \mathrm{~mm}$ and having a width $2 a$ of the same order. As a consequence a vortex patch formed along the edge of the plate, with diameter roughly equal to its width, affecting wave generation significantly. Several decades later, Peacock et al. (2008) repeated these investigations in a more controlled setting, using a thin plate of height $b=16.5 \mathrm{~mm}$ and width $2 a=1.28 \mathrm{~mm}$, mounted on a rigid bottom in a fluid of buoyancy frequency $N=1.18 \mathrm{~s}^{-1}$ and kinematic viscosity $\nu=1.10 \mathrm{~mm}^{2} \mathrm{~s}^{-1}$. The oscillations had amplitude $A=0.88 \mathrm{~mm}$ and frequency $\omega_{0}=0.836 \mathrm{~s}^{-1}$, yielding Stokes number $S t=\omega_{0} b^{2} / \nu=210$ and Keulegan-Carpenter number $K e=A / b=0.05$.

The high aspect ratio $b / a \approx 26$ allows the plate to be considered as a knife edge, while the presence of the rigid bottom adds the image of the edge through the line $z=0$, hence turning the plate into the limit as $a \rightarrow 0$ of the elliptic cylinder. Its representation follows as

$$
f(x, z)=-2 \mathrm{i} U \tan \theta_{0} \delta^{\prime}(x) H(b-|z|)\left(b^{2}-z^{2}\right)^{1 / 2},
$$


Near-field internal wave beams in two dimensions
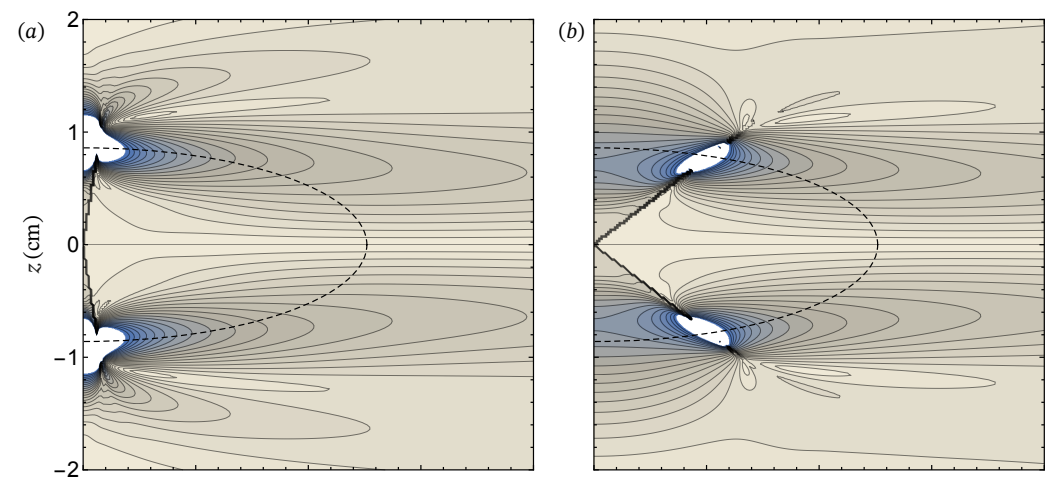

(c)
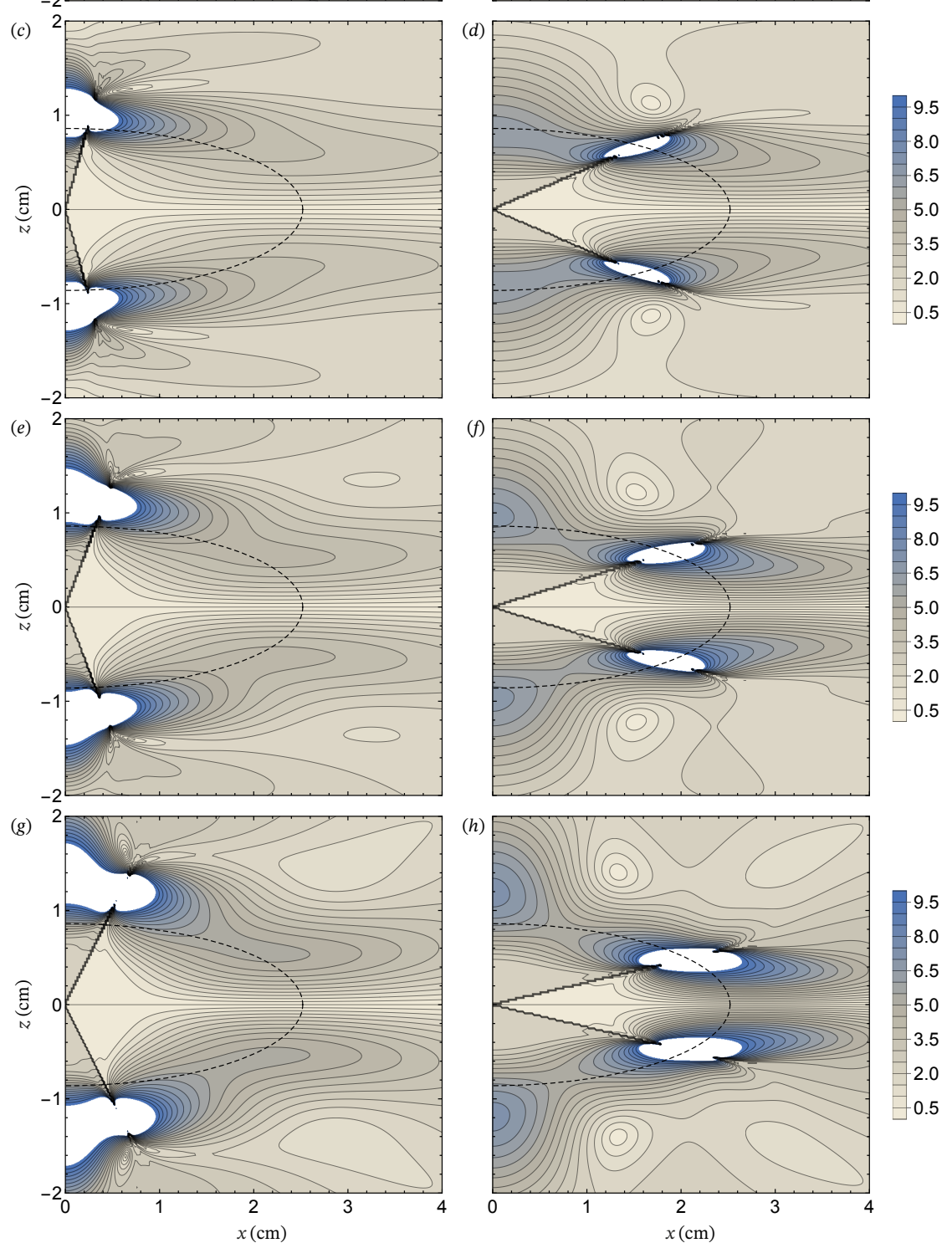

FIGURE 6. Near field for figure 5 , at $(a, b) \omega_{0} / N=0.15,(c, d) \omega_{0} / N=0.26,(e, f) \omega_{0} / N=0.35$ and $(g, h) \omega_{0} / N=0.44$, using $(a, c, e, g)$ the classical theory $(8.25)$ and $(b, d, f, h)$ the present theory $(8.21)$. The waves are calculated both inside and outside the cylinder, whose outline is shown dashed. 


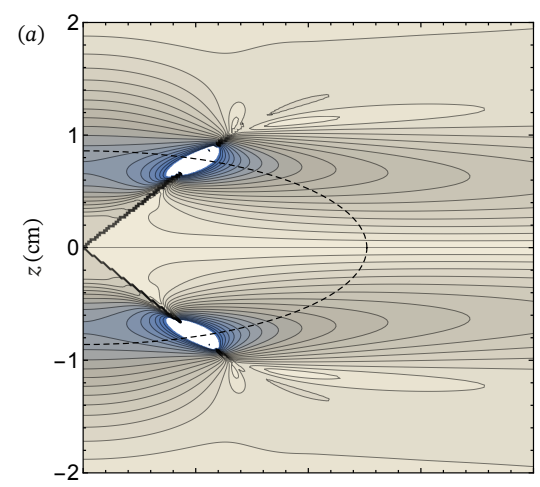

(b)
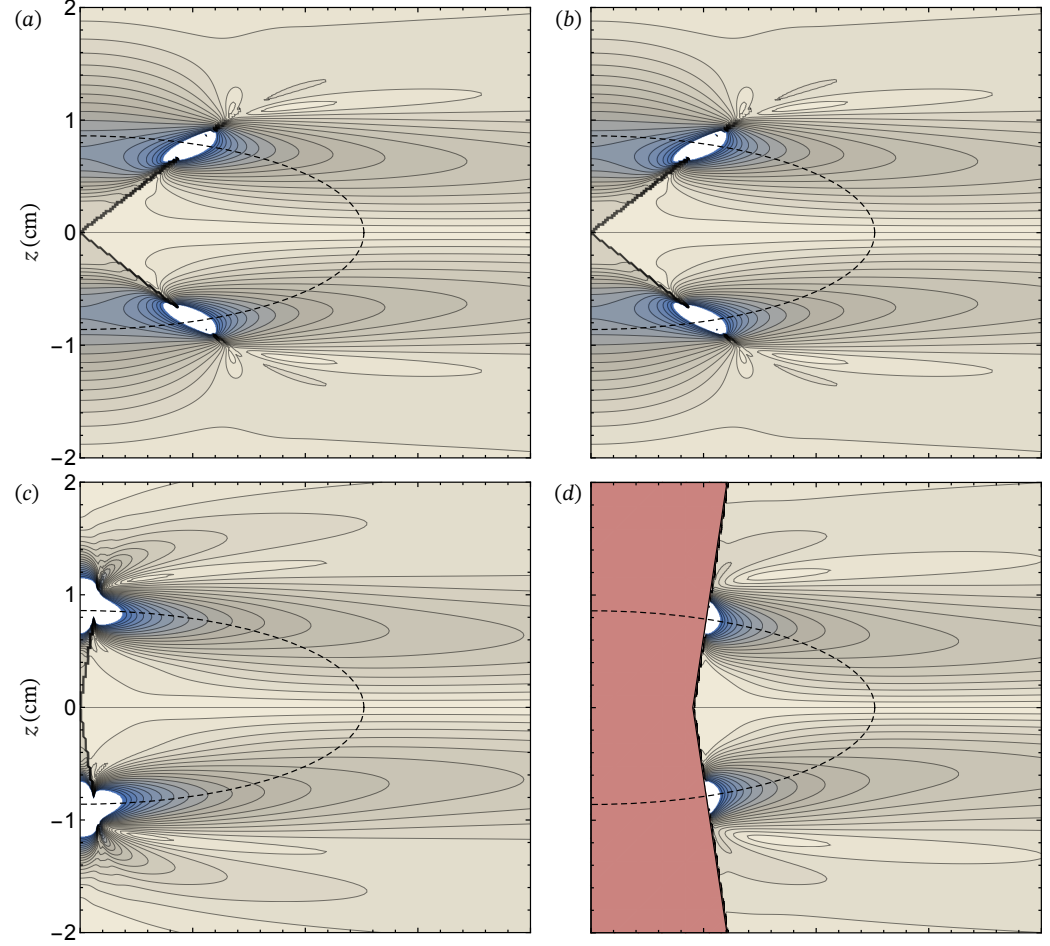

(e)

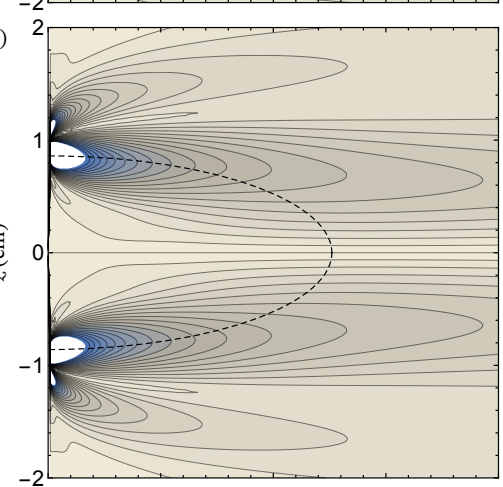

(d)

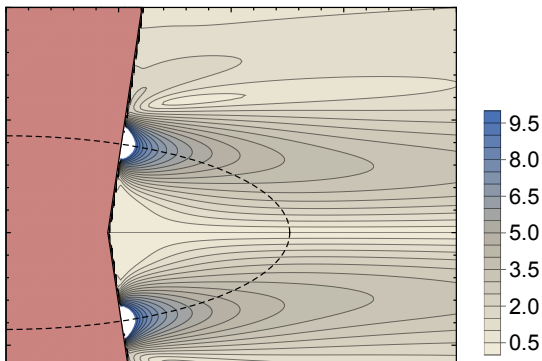

(g)
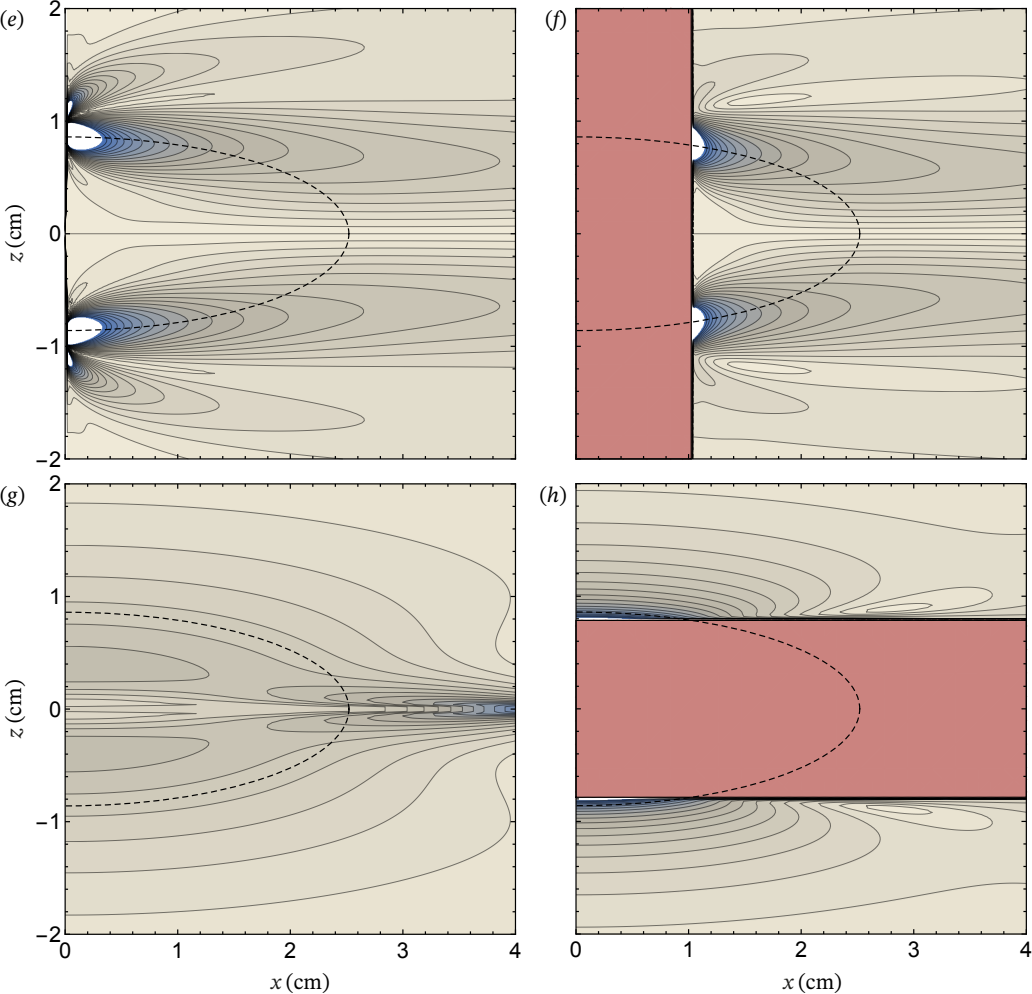

(h)

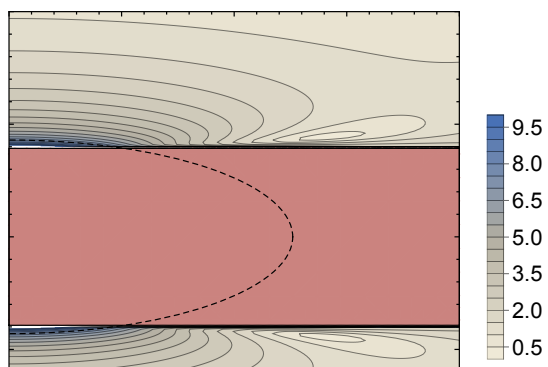

FIGURE 7. Near field for figure 5(a), using (8.21) in $(a, b),(8.22)$ in $(c, d),(8.23)$ in $(e, f)$ and $(8.24)$ in $(g, h)$, ignoring the viscous correction in the argument of the Bessel function $\mathrm{J}_{1}$ in $(a, c, e, g)$ and taking it into account in $(b, d, f, h)$. The regions excluded by the conditions (8.9), (8.10) and (8.11) for the convergence of the integrals are shown in red in $(d),(f)$ and $(h)$, respectively. 
Near-field internal wave beams in two dimensions
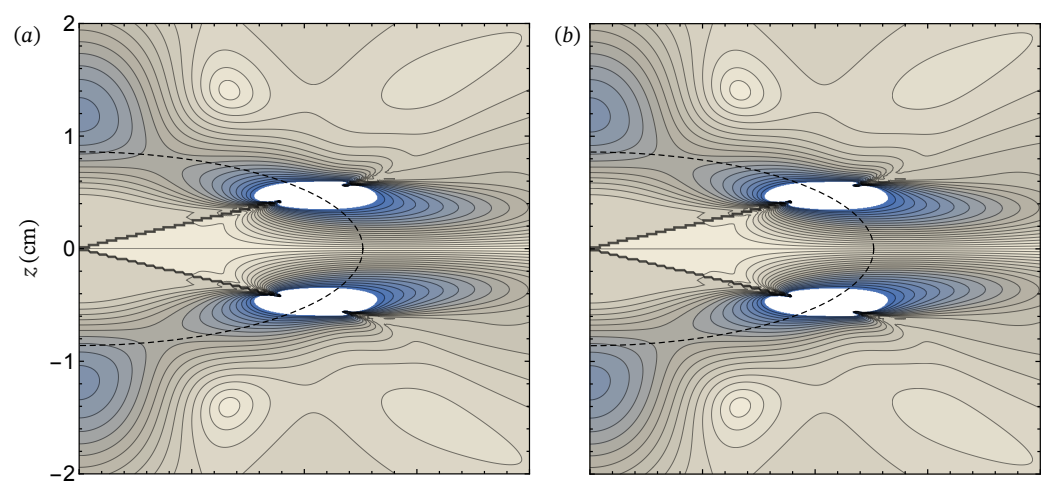

(c)

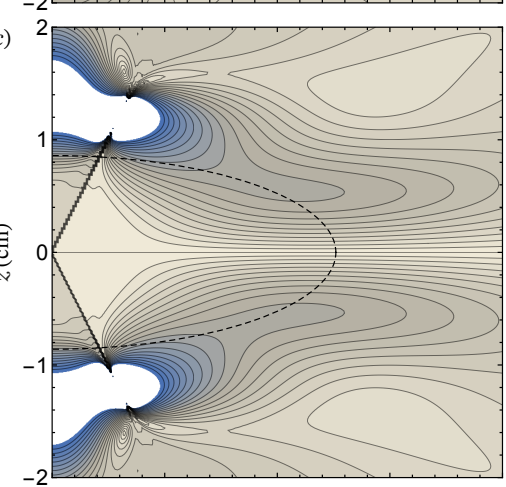

(d)

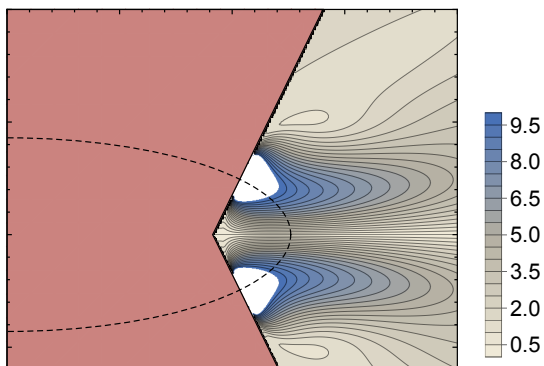

(e)

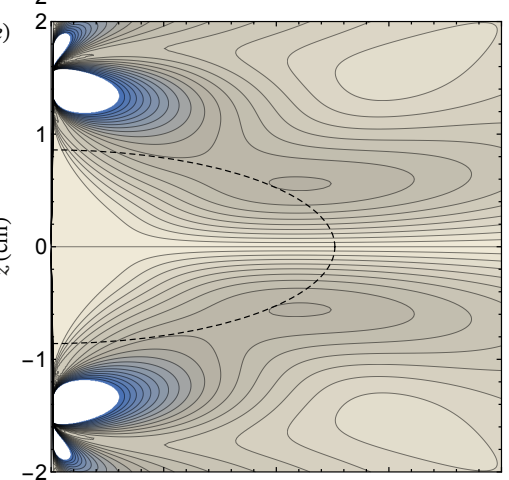

(g)
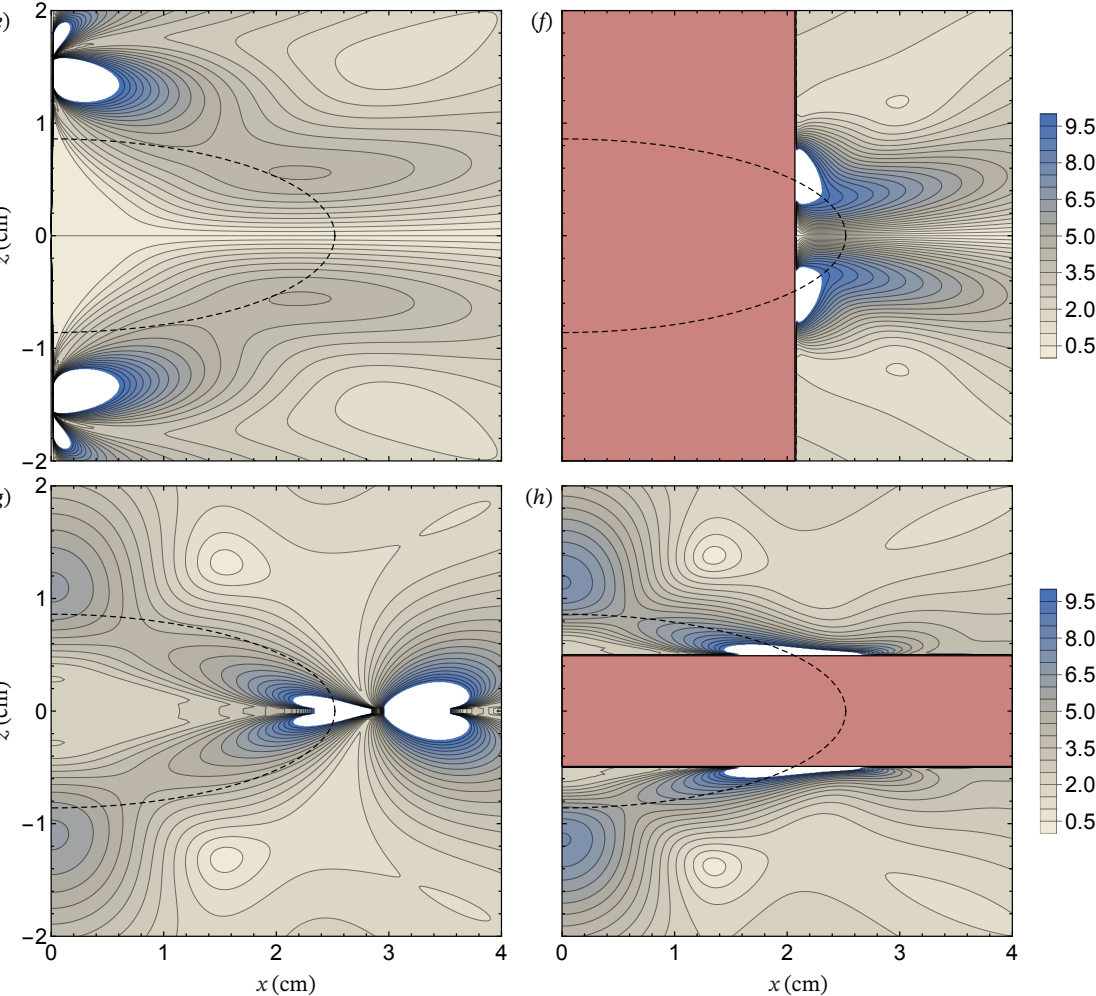

(h)

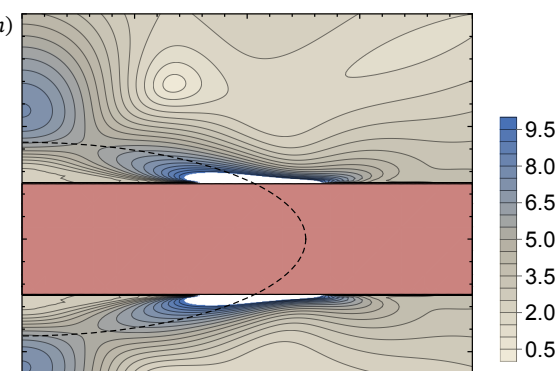

FIGURE 8. Near field for figure 5(d). The mode of representation is the same as in figure 7. 
with spectrum

$$
f(k, m)=2 \pi b U \tan \theta_{0} \frac{k}{m} \mathrm{~J}_{1}(m b) .
$$

The theory of $\S 5.2$ gives waves

$$
\begin{aligned}
\boldsymbol{u}=b U \exp \left(-\mathrm{i} \omega_{0} t\right) \int_{0}^{\infty} \mathrm{J}_{1}\left(\kappa b \sin \theta_{0}\right) \exp \left(-\beta \kappa^{3}|x| / \sin \theta_{0}\right) \exp \left(\mathrm{i} \kappa|x| \cos \theta_{0}\right) \\
\quad \times\left[\boldsymbol{e}_{x} \cos \left(\kappa z \sin \theta_{0}\right) \sin \theta_{0}-\mathrm{i} \boldsymbol{e}_{z} \sin \left(\kappa z \sin \theta_{0}\right) \cos \theta_{0} \operatorname{sign} x\right] \mathrm{d} \kappa,
\end{aligned}
$$

and a boundary layer

$$
\boldsymbol{u}_{\mathrm{b}}=\mathrm{i} U \boldsymbol{e}_{z} \exp \left(-\mathrm{i} \omega_{0} t\right) \frac{\operatorname{sign} x}{\sin \theta_{0} \cos \theta_{0}} H(b-|z|) \frac{z}{\left(b^{2}-z^{2}\right)^{1 / 2}} \exp \left(-k_{\mathrm{b}}|x|\right) \exp \left(-\mathrm{i} k_{\mathrm{b}}|x|\right),
$$

with $k_{\mathrm{b}}$ as in (5.21), while the classical theory of Hurley \& Keady (1997) gives waves

$$
\begin{aligned}
\boldsymbol{u}_{\mathrm{c}}=\frac{b U}{2} \exp \left(-\mathrm{i} \omega_{0} t\right) & \sum_{ \pm}\left(\boldsymbol{e}_{x} \sin \theta_{0} \pm \boldsymbol{e}_{z} \cos \theta_{0}\right) \\
& \times \int_{0}^{\infty} \mathrm{J}_{1}\left(\kappa b \sin \theta_{0}\right) \exp \left(-\beta \kappa^{3}\left|z_{ \pm}\right|\right) \exp \left( \pm \mathrm{i} \kappa x_{ \pm} \operatorname{sign} z_{ \pm}\right) \mathrm{d} \kappa
\end{aligned}
$$

and no boundary layer.

Peacock et al. (2008) used synthetic schlieren to measure the buoyancy frequency disturbance $\Delta N^{2}=-\mathrm{i}\left(N^{2} / \omega_{0}\right)(\partial w) /(\partial z)$, and compared the outcome with the predictions of Hurley \& Keady (1997). Choosing, as they did, the phase of the oscillation $\phi=\omega_{0} t$ to be zero at the instant when the plate moves right to left through the midpoint of its oscillation, we write the position of the plate as $\operatorname{Re}\left[-\mathrm{i} A \boldsymbol{e}_{x} \exp \left(-\mathrm{i} \omega_{0} t\right)\right]$, so that $U=$ $-\omega_{0} A$. The present theory gives waves

$$
\begin{aligned}
& \frac{\Delta N^{2}}{N^{2}}=b A \exp \left(-\mathrm{i} \omega_{0} t\right) \sin \theta_{0} \cos \theta_{0} \operatorname{sign} x \int_{0}^{\infty} \kappa \mathrm{J}_{1}\left(\kappa b \sin \theta_{0}\right) \\
& \times \exp \left(-\beta \kappa^{3}|x| / \sin \theta_{0}\right) \exp \left(\mathrm{i} \kappa|x| \cos \theta_{0}\right) \cos \left(\kappa z \sin \theta_{0}\right) \mathrm{d} \kappa,
\end{aligned}
$$

and a boundary layer

$$
\left(\frac{\Delta N^{2}}{N^{2}}\right)_{\mathrm{b}}=-\frac{A}{b} \exp \left(-\mathrm{i} \omega_{0} t\right) \frac{\operatorname{sign} x}{\sin \theta_{0} \cos \theta_{0}} \frac{H(b-|z|)}{\left[1-(z / b)^{2}\right]^{3 / 2}} \exp \left(-k_{\mathrm{b}}|x|\right) \exp \left(-\mathrm{i} k_{\mathrm{b}}|x|\right),
$$

while the Hurley-Keady theory gives only waves

$$
\begin{aligned}
\left(\frac{\Delta N^{2}}{N^{2}}\right)_{\mathrm{c}}=\frac{b A}{2} \exp ( & \left.-\mathrm{i} \omega_{0} t\right) \sin \theta_{0} \cos \theta_{0} \sum_{ \pm}( \pm) \operatorname{sign} z_{ \pm} \\
& \times \int_{0}^{\infty} \kappa \mathrm{J}_{1}\left(\kappa b \sin \theta_{0}\right) \exp \left(-\beta \kappa^{3}\left|z_{ \pm}\right|\right) \exp \left( \pm \mathrm{i} \kappa x_{ \pm} \operatorname{sign} z_{ \pm}\right) \mathrm{d} \kappa
\end{aligned}
$$

As for the elliptic cylinder in $\S 8.1$, the transverse profiles at cross sections $z_{+}=3 b$ and $10 \mathrm{~b}$ in figure 7 of Peacock et al. (2008) belong to the far field, where the present theory and the Hurley-Keady theory exhibit no significant difference. We consider instead the contour map in their figure 6, and plot the outcome of the present theory in figure 9 . The agreement with experiment is good, especially in the far field. A close-up of the near field is shown in figure 10: the discontinuity of the Hurley-Keady theory across the 


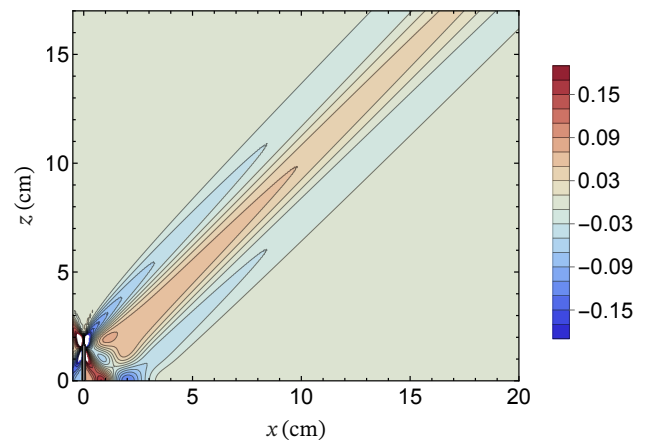

FIGURE 9. Contour map of $\Delta N^{2}$ (in s $\mathrm{s}^{-2}$ ) at phase $\phi=0$, as predicted by (8.31), for the vertical barrier in figure 6 of Peacock et al. (2008). The waves propagate at the angle $\theta_{0}=45^{\circ}$ to the vertical. The white areas correspond to off-scale values of the plotted quantity.
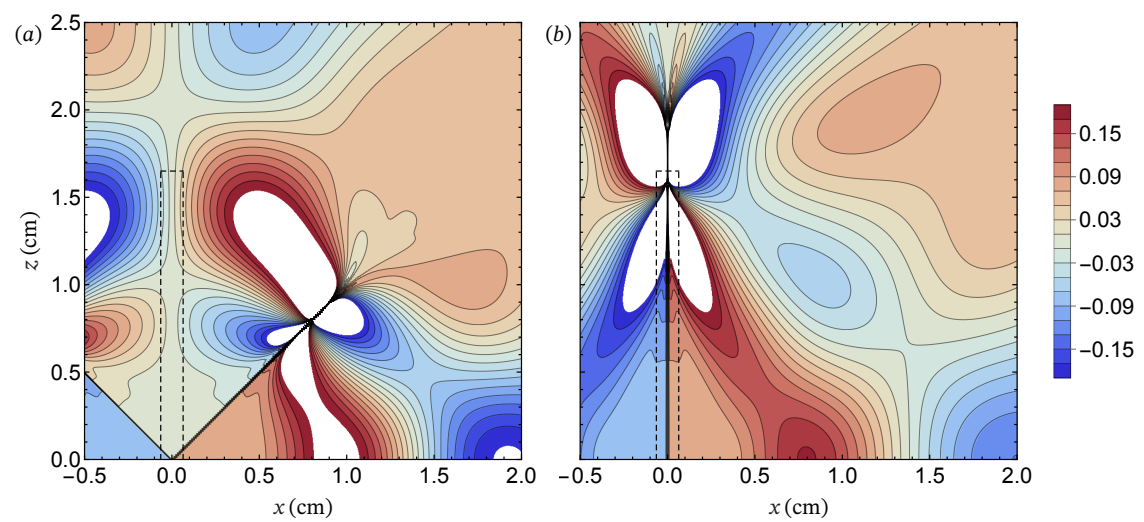

FIGURE 10. Near field for figure 9, using (a) the classical theory (8.33) and (b) the present theory (8.31). The outline of the barrier is shown dashed.

portion $\left|x_{ \pm}\right|<b \sin \theta_{0}$ of the beam separation lines $z_{ \pm}=0$ is strikingly visible, whereas the present theory keeps the discontinuity at the knife edge $(x=0,0<z<b)$.

Figure 11 shows the effect of adding the boundary-layer (8.32): singularities spread through the fluid at the level $z=b$ of the tip of the knife edge. This unphysical behaviour illustrates the singular nature of the low-viscosity limit $S t \rightarrow \infty$, such that no free-slip regime is reached at the horizontal line $z=b$ through the tip; there, however large $S t$ can be, a no-slip solution of the equations of motion is required. The interested reader may check that the same inverse square root singularity of the velocity is obtained for the broadside oscillations of a horizontal disc at the vertical cylinder through the rim of the disc, when the large-St behaviour of the no-slip solution of Davis \& Llewellyn Smith (2010) is considered.

\subsection{Wave generator}

A major breakthrough for the laboratory study of internal waves has been the design of a wave generator by Gostiaux et al. (2007) and Mercier et al. (2010), allowing the imposition of an arbitrary wave profile at a plane boundary. In the original design, the profile was discretized as a camshaft-driven stack of plates; this design has since been improved and applied worldwide to a variety of problems, such as those described in the reviews by Dauxois et al. (2018) and Sibgatullin \& Ermanyuk (2019). Alternative designs have also been developed, in which each generating element, either plate, bar or rod, is 

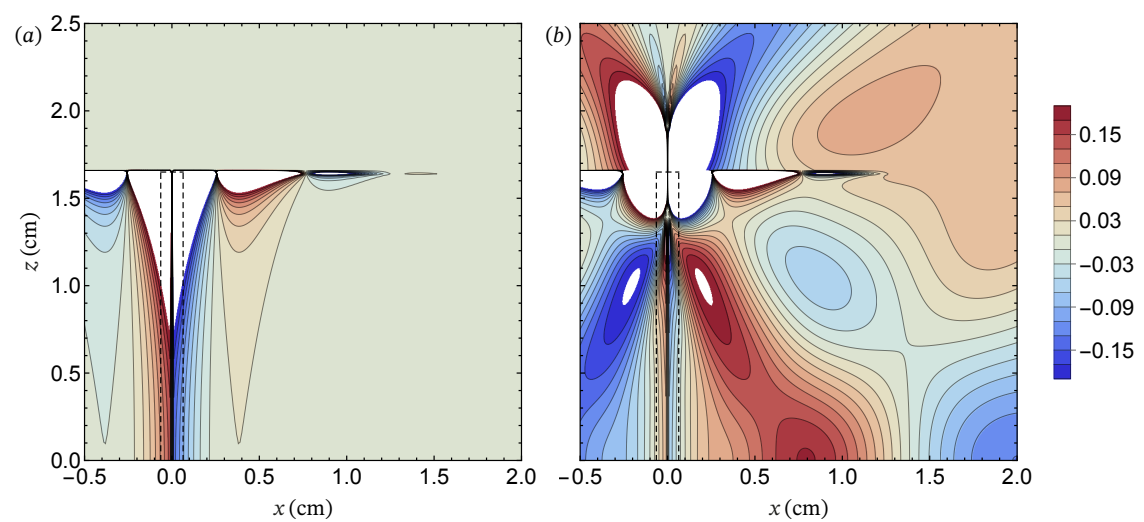

FIGURE 11. Contour maps of $\Delta N^{2}$ (in s ${ }^{-2}$ ) for the boundary layer (8.32), shown either (a) in isolation or $(b)$ in combination with the waves (8.31), in the same conditions as for figure 10.

controlled by an individual motor. In one design, called GOAL (Generator of Oscillations As you Like), the elements are in direct contact with the fluid (Dossmann et al. 2016, 2017; Brunet, Dauxois \& Cortet 2019); in another, called ASWaM (Arbitrary Spectrum Wave Maker), they operate behind a neoprene sheet smoothing out the discretization (Dobra, Lawrie \& Dalziel 2019).

Imposition of the velocity profile $w_{0}\left(x_{0}\right)$ on the positive side of a line $z_{0}=0$, inclined at the angle $\varphi_{0}$ to the horizontal, and addition, on the negative side, of the image of the profile through this line, so as to transform the problem into one over the whole plane, yield a velocity discontinuity $2 w_{0}\left(x_{0}\right)$ at $z_{0}=0$, hence a source of mass

$$
f_{0}\left(x_{0}, z_{0}\right)=2 w_{0}\left(x_{0}\right) \delta\left(z_{0}\right), \quad f_{0}\left(k_{0}, m_{0}\right)=2 w_{0}\left(k_{0}\right) .
$$

Both waves and a boundary layer are produced, given by the formulae of $\S 5$. According to them, the boundary layer is of the same order as the waves when the generator is inclined, and negligible compared with the waves when the generator is horizontal or vertical. As previously discussed, such prediction of a boundary layer based on a free-slip boundary condition is questionable. Beckebanze et al. (2019) discussed the appropriate condition at a vertical wave generator and concluded that the boundary layer may be neglected and a free-slip condition used.

One of the experiments by Mercier et al. (2010) aimed at reproducing the self-similar wave beam, propagating downward to the right, generated in a viscous fluid by a point dipole source at $\left(x_{-}=0, z_{-}=l\right)$. In this beam, calculated by Thomas \& Stevenson (1972) and Machicoane et al. (2015), the fluid velocity writes

$$
\boldsymbol{u}_{\mathrm{c}}=-\frac{3 U}{\Gamma(2 / 3)} \boldsymbol{e}_{z_{-}} \exp \left(-\mathrm{i} \omega_{0} t\right)\left(\frac{l}{l-z_{-}}\right)^{2 / 3}\left(c_{2}+\mathrm{i} s_{2}\right)\left\{\frac{x_{-}}{\left[\beta\left(l-z_{-}\right)\right]^{1 / 3}}\right\},
$$

where the real functions $c_{\mu}$ and $s_{\mu}$, defined as

$$
\left(c_{\mu}+\mathrm{is}_{\mu}\right)(x)=\int_{0}^{\infty} k^{\mu-1} \exp \left(-k^{3}\right) \exp (\mathrm{i} k x) \mathrm{d} k
$$

and such that $\left(c_{\mu}+\mathrm{i} s_{\mu}\right)(0)=\Gamma(\mu / 3) / 3$, have been introduced independently by Moore \& Saffman (1969) for rotating fluids and Thomas \& Stevenson (1972) for stratified fluids, with different notations, and their properties studied in greater detail by Voisin (2003) and Le Dizès \& Le Bars (2017).

The aim of the experiment was to impose the profile (8.35) along the line $z_{-}=0$, 
setting this profile such that the distance $l$ to the virtual source was large enough for the beam to have reached self-similarity already, and then to check whether the resulting waves evolved according to (8.35). To avoid having to reconfigure the generator to be along the line $z_{-}=0$ for each frequency of oscillation, the generator was positioned vertically and the profile $u(x=0, z)=-\boldsymbol{u}_{\mathrm{c}}\left(x_{-}=z, z_{-}=0\right) \cdot \boldsymbol{e}_{z_{-}}$imposed along it, assuming the angle of propagation $\pi / 2-\theta_{0}$ to the horizontal to be small enough for the approximation to be valid. The forcing becomes

$$
g(z)=\frac{4 U}{\Gamma(2 / 3)}\left(c_{2}+\mathrm{i} s_{2}\right)\left[\frac{z}{(\beta l)^{1 / 3}}\right], \quad g(m)=\frac{12 \pi U}{\Gamma(2 / 3)}(\beta l)^{2 / 3} H(m) m \exp \left(-\beta l m^{3}\right),
$$

and the waves follow as

$$
\boldsymbol{u}=-\frac{3 U}{\Gamma(2 / 3)} \boldsymbol{e}_{z_{-}} \exp \left(-\mathrm{i} \omega_{0} t\right) \frac{l^{2 / 3} \sin ^{5 / 3} \theta_{0}}{\left(x+l \sin ^{4} \theta_{0}\right)^{2 / 3}}\left(c_{2}+\mathrm{i} s_{2}\right)\left\{x_{-}\left[\frac{\sin \theta_{0}}{\beta\left(x+l \sin ^{4} \theta_{0}\right)}\right]^{1 / 3}\right\}
$$

The experiment took place in a fluid of buoyancy frequency $N=0.82 \mathrm{~s}^{-1}$. The kinematic viscosity was not specified and has been taken as $v=1 \mathrm{~mm}^{2} \mathrm{~s}^{-1}$. The virtual origin was at $l=44 \mathrm{~cm}$, determined from a fit to figure 2(a) of Mercier et al. (2010), and the amplitude of oscillation was $A=10 \mathrm{~mm}$. The phase of the oscillation $\phi=\omega_{0} t$ was chosen to be zero at the instant when the central plate of the generator was at the rightmost point of its oscillation, yielding $\operatorname{Re}\left[A \boldsymbol{e}_{x} \exp \left(-i \omega_{0} t\right)\right]$ for the position of the plate, so that $U=-\mathrm{i} \omega_{0} A$. Matching numerical simulations were also performed.

The plotted quantity was the buoyancy disturbance $B=-g \rho / \rho_{0}=-\mathrm{i}\left(N^{2} / \omega_{0}\right) w$, for which the Thomas-Stevenson approach gives

$$
\frac{B_{\mathrm{c}}}{N^{2} A}=\frac{3 \cos \theta_{0}}{\Gamma(2 / 3)} \exp \left(-\mathrm{i} \omega_{0} t\right)\left(\frac{l}{l-z_{-}}\right)^{2 / 3}\left(c_{2}+\mathrm{i} s_{2}\right)\left\{\frac{x_{-}}{\left[\beta\left(l-z_{-}\right)\right]^{1 / 3}}\right\},
$$

and the present approach gives

$$
\frac{B}{N^{2} A}=\frac{3 \cos \theta_{0}}{\Gamma(2 / 3)} \exp \left(-\mathrm{i} \omega_{0} t\right) \frac{l^{2 / 3} \sin ^{5 / 3} \theta_{0}}{\left(x+l \sin ^{4} \theta_{0}\right)^{2 / 3}}\left(c_{2}+\mathrm{i} s_{2}\right)\left\{x_{-}\left[\frac{\sin \theta_{0}}{\beta\left(x+l \sin ^{4} \theta_{0}\right)}\right]^{1 / 3}\right\}
$$

Figures 12 and 13 of Mercier et al. (2010) present measurements for oscillations at the frequency $\omega_{0}=0.20 \mathrm{~s}^{-1}$, corresponding to propagation at $14^{\circ}$ to the horizontal. The transverse profiles in their figure 13, measured at eleven cross sections every $2 \mathrm{~cm}$ from $z_{-}=-3 \mathrm{~cm}$ to $z_{-}=-23 \mathrm{~cm}$, are all in the far field; this is because, with an across-beam distance to the beam axis of at most $4.5 \mathrm{~cm}$, corresponding to half the active region of the generator, and an along-beam distance to the virtual origin of at least $44 \mathrm{~cm}$, we are already in the far field at the generator. As the result, the present theory is indistinguishable from the Thomas-Stevenson theory, considered by Mercier et al. (2010), at those cross sections. This legitimates a posteriori the use of a vertical generator.

We focus instead on their figure 12, and present matching contour maps in the present figure 12. The Thomas-Stevenson theory and the present theory only differ from each other in the close vicinity of the generator, the latter giving slightly larger values. The theory agrees with both experiments and simulations, the agreement being better with the latter owing to the more controlled numerical conditions.

\subsection{Thin topography}

The main manifestation of monochromatic internal waves in the environment is the internal or baroclinic tide, generated in the ocean by the oscillation of the barotropic tide 

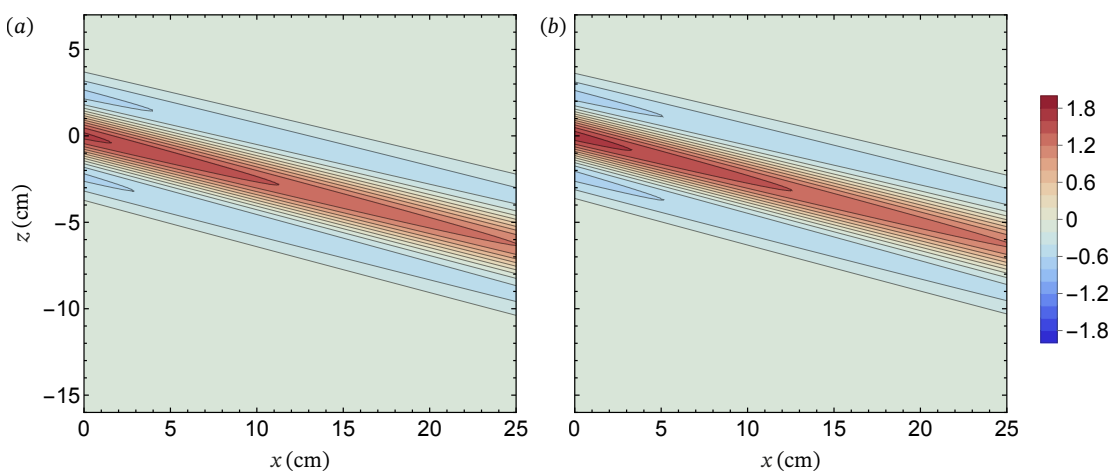

FIGURE 12. Contour maps of $B\left(\right.$ in $\left.\mathrm{mm} \mathrm{s}^{-2}\right)$ at phase $\phi=0$ for the wave generator in the 'wave beam' experiment in figure 12 of Mercier et al. (2010), using (a) the Thomas-Stevenson profile (8.39) and (b) the present profile (8.40).

over bottom topography (Garrett \& Kunze 2007). A convenient approximation, sometimes called 'weak topography', is the concept of a thin topography, that is a topography of infinitesimal slope. It has been introduced by Cox \& Sandstrom (1962), developed by Bell $(1975 a, b)$ and Llewellyn Smith \& Young (2002), and applied to the global calculation of the internal tide by St. Laurent \& Garrett (2002), Nycander (2005), Melet et al. (2013), Falahat et al. (2014) and Vic et al. (2019).

The approximation requires the topographic slope to be small compared with the slope of the wave rays. Topographies are said subcritical if their slope is everywhere smaller than the slope of the rays, and supercritical otherwise. For two-dimensional topography of profile $h(x)$, Balmforth et al. (2002) introduced a 'criticality parameter'

$$
\varepsilon=\frac{\max \left|h^{\prime}(x)\right|}{\cot \theta_{0}},
$$

and studied the validity of the approximation, in theory $\varepsilon \ll 1$, as $\varepsilon$ increases from 0 to 1 for a variety of subcritical topographies. Among those was an isolated Gaussian bump. In this respect, the elliptic cylinder of $\S 8.1$ and the vertical barrier of $\S 8.2$ are extreme examples of supercritical topographies for which $\varepsilon \rightarrow \infty$.

Consider the problem in the frame of reference of the barotropic tide. The topography oscillates at the velocity $(U, 0) \exp \left(-i \omega_{0} t\right)$ in an otherwise quiescent fluid with rigid bottom at $z=0$. Assuming the topography to be thin, the free-slip boundary condition simplifies to $w=-U h^{\prime}(x)$ at $z=0$. Adding the image of the topography through the bottom, the representation of the forcing follows as

$$
f(x, z)=-2 U h^{\prime}(x) \delta(z), \quad f(k, m)=-2 \mathrm{i} U k h(k),
$$

yielding the internal tide

$$
\begin{aligned}
\boldsymbol{u}= & -\mathrm{i} \frac{U}{2 \pi} \exp \left(-\mathrm{i} \omega_{0} t\right) \cos \theta_{0} \sum_{ \pm}\left(\boldsymbol{e}_{x} \sin \theta_{0} \pm \boldsymbol{e}_{z} \cos \theta_{0}\right) \\
& \times \int_{0}^{\infty} \kappa h\left(k= \pm \kappa \cos \theta_{0} \operatorname{sign} z\right) \exp \left(-\beta \kappa^{3}|z| / \cos \theta_{0}\right) \exp \left( \pm \mathrm{i} \kappa x_{ \pm} \operatorname{sign} z\right) \mathrm{d} \kappa
\end{aligned}
$$

As already discussed, the boundary layer is negligible. For the same forcing, the classical 
theory of $\S 2$ gives

$$
\begin{aligned}
\boldsymbol{u}_{\mathrm{c}}=-\mathrm{i} & \frac{U}{2 \pi} \exp \left(-\mathrm{i} \omega_{0} t\right) \cos \theta_{0} \sum_{ \pm}\left(\boldsymbol{e}_{x} \sin \theta_{0} \pm \boldsymbol{e}_{z} \cos \theta_{0}\right) \\
& \times \int_{0}^{\infty} \kappa h\left(k= \pm \kappa \cos \theta_{0} \operatorname{sign} z_{ \pm}\right) \exp \left(-\beta \kappa^{3}\left|z_{ \pm}\right|\right) \exp \left( \pm \mathrm{i} \kappa x_{ \pm} \operatorname{sign} z_{ \pm}\right) \mathrm{d} \kappa
\end{aligned}
$$

Applied to the Gaussian bump

$$
h(x)=h_{0} \exp \left(-\frac{x^{2}}{2 a^{2}}\right), \quad h(k)=(2 \pi)^{1 / 2} a h_{0} \exp \left(-\frac{k^{2} a^{2}}{2}\right),
$$

these results give, for the present theory,

$$
\begin{aligned}
\boldsymbol{u}= & \left(\frac{2}{\pi}\right)^{1 / 2} a h_{0} U \exp \left(-\mathrm{i} \omega_{0} t\right) \cos \theta_{0} \\
& \times \int_{0}^{\infty} \kappa \exp \left(-\kappa^{2} a^{2} \cos ^{2} \theta_{0} / 2\right) \exp \left(-\beta \kappa^{3}|z| / \cos \theta_{0}\right) \exp \left(-\mathrm{i} \kappa|z| \sin \theta_{0}\right) \\
& \times\left[\boldsymbol{e}_{z} \cos \theta_{0} \sin \left(\kappa x \cos \theta_{0}\right) \operatorname{sign} z-\mathrm{i} \boldsymbol{e}_{x} \sin \theta_{0} \cos \left(\kappa x \cos \theta_{0}\right)\right] \mathrm{d} \kappa
\end{aligned}
$$

and for the classical theory,

$$
\begin{aligned}
\boldsymbol{u}_{\mathrm{c}}=-\mathrm{i} \frac{a h_{0} U}{(2 \pi)^{1 / 2}} \exp \left(-\mathrm{i} \omega_{0} t\right) \cos \theta_{0} \sum_{ \pm}\left(\boldsymbol{e}_{x} \sin \theta_{0} \pm \boldsymbol{e}_{z} \cos \theta_{0}\right) \\
\quad \times \int_{0}^{\infty} \kappa \exp \left(-\kappa^{2} a^{2} \cos ^{2} \theta_{0} / 2\right) \exp \left(-\beta \kappa^{3}\left|z_{ \pm}\right|\right) \exp \left( \pm \mathrm{i} \kappa x_{ \pm} \operatorname{sign} z_{ \pm}\right) \mathrm{d} \kappa
\end{aligned}
$$

on the assumption that the criticality parameter $\varepsilon=\left(h_{0} \tan \theta_{0}\right) /(a \sqrt{e})$ is small.

In the inviscid case, the Fourier transform

$$
\int_{0}^{\infty} k \exp \left(-k^{2}\right) \exp (\mathrm{i} k x) \mathrm{d} k=\frac{1}{2}\left[1-x F\left(\frac{x}{2}\right)\right]+\mathrm{i} \frac{\pi^{1 / 2}}{4} x \exp \left(-\frac{x^{2}}{4}\right),
$$

where $F(x)=\mathrm{e}^{-x^{2}} \int_{0}^{x} \mathrm{e}^{t^{2}} \mathrm{~d} t$ is Dawson's integral, taken from table 5 of Voisin (2003), yields

$$
\begin{aligned}
& \boldsymbol{u}_{\mathrm{c}}=-\mathrm{i} \frac{h_{0}}{a \cos \theta_{0}} \frac{U}{(2 \pi)^{1 / 2}} \exp \left(-\mathrm{i} \omega_{0} t\right) \sum_{ \pm}\left(\boldsymbol{e}_{x} \sin \theta_{0} \pm \boldsymbol{e}_{z} \cos \theta_{0}\right) \\
& \times\left[1-\frac{\sqrt{2 x_{ \pm}}}{a \cos \theta_{0}} F\left(\frac{x_{ \pm}}{\sqrt{2 a \cos \theta_{0}}}\right) \pm \mathrm{i}\left(\frac{\pi}{2}\right)^{1 / 2} \frac{x_{ \pm}}{a \cos \theta_{0}} \exp \left(-\frac{x_{ \pm}^{2}}{2 a^{2} \cos ^{2} \theta_{0}}\right) \operatorname{sign} z_{ \pm}\right] .
\end{aligned}
$$

Accordingly, the classical solution (8.47) is discontinuous across the entirety of the lines $z_{ \pm}=0$, while the discontinuity of the present solution (8.46) is limited to the forcing line $z=0$ where it is of no consequence. In practice, given the rapid decay of $x \exp \left(-x^{2} / 2\right)$ past its maximum at $x=1$, such that the function is already negligible at $x=4$ say, the discontinuity of the classical solution is only visible for $\left|x_{ \pm}\right| \lesssim 4 a \cos \theta_{0}$.

In the same series of experiments as for the vertical barrier in $\S 8.2$, Peacock et al. (2008) considered a Gaussian bump of height $h_{0}=14.7 \mathrm{~mm}$ and standard deviation $a=$ $20 \mathrm{~mm}$, hence maximum slope angle of $24^{\circ}$, oscillating with amplitude $A=2.79 \mathrm{~mm}$, hence Keulegan-Carpenter number $\mathrm{Ke}=A / a=0.14$, in a fluid of kinematic viscosity $v=1.10 \mathrm{~mm}^{2} \mathrm{~s}^{-1}$. Two different criticality parameters were obtained by varying the 

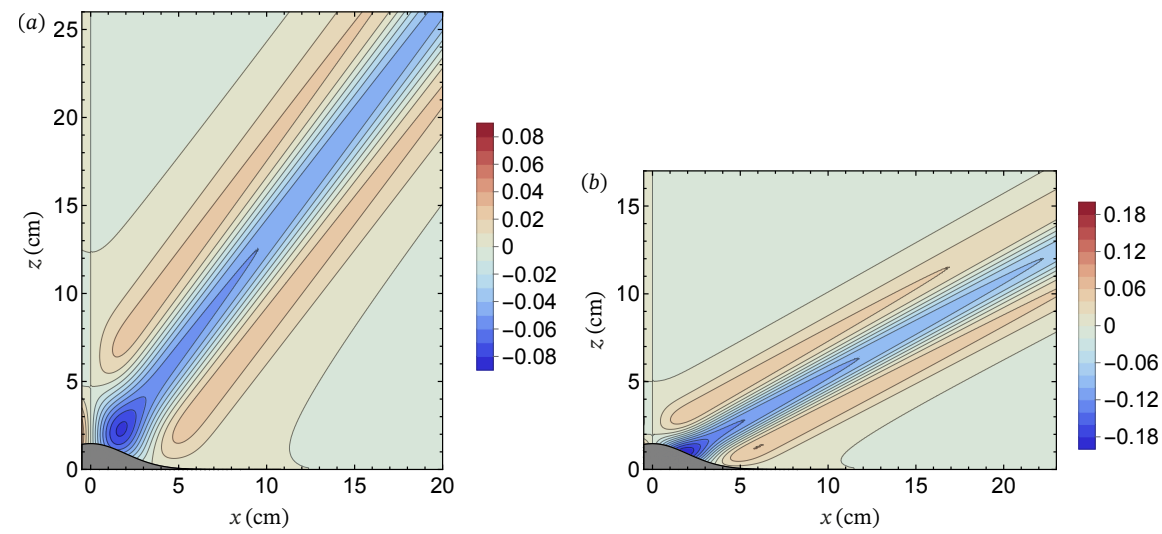

FIGURE 13. Contour maps of $\Delta N^{2}\left(\right.$ in s $^{-2}$ ) at phase $\phi=\pi / 2$, as predicted by (8.50), for the oscillations at relative frequencies $(a) \omega_{0} / N=0.80$ and $(b) \omega_{0} / N=0.48$ of the Gaussian bump in figures 2 and 4 , respectively, of Peacock et al. (2008). The waves propagate at the angles $(a) \theta_{0}=37^{\circ}$ and $(b) \theta_{0}=62^{\circ}$ to the vertical, with criticality parameters $(a) \varepsilon=0.34$ and $(b) \varepsilon=0.82$.

frequency of oscillation: $\epsilon=0.34$, for $\omega_{0}=0.98 \mathrm{~s}^{-1}$ and $N=1.23 \mathrm{~s}^{-1}$; and $\epsilon=0.82$, for $\omega_{0}=0.59 \mathrm{~s}^{-1}$ and $N=1.24 \mathrm{~s}^{-1}$. The associated Stokes numbers $S t=\omega_{0} a^{2} / \nu$ were 360 and 210 , respectively.

The measured quantity was, again, the buoyancy frequency disturbance, compared with an extension of the theory of Balmforth et al. (2002). Choosing the phase origin as in $\S 8.2$, so that $U=-\omega_{0} A$, the present theory gives

$$
\begin{aligned}
\frac{\Delta N^{2}}{N^{2}}=\left(\frac{2}{\pi}\right)^{1 / 2} a h_{0} A & \exp \left(-\mathrm{i} \omega_{0} t\right) \sin \theta_{0} \cos ^{2} \theta_{0} \int_{0}^{\infty} \kappa^{2} \exp \left(-\kappa^{2} a^{2} \cos ^{2} \theta_{0} / 2\right) \\
& \times \exp \left(-\beta \kappa^{3}|z| / \cos \theta_{0}\right) \sin \left(\kappa x \cos \theta_{0}\right) \exp \left(-\mathrm{i} \kappa|z| \sin \theta_{0}\right) \mathrm{d} \kappa
\end{aligned}
$$

while the classical theory gives

$$
\begin{aligned}
\left(\frac{\Delta N^{2}}{N^{2}}\right)_{\mathrm{c}}= & -\mathrm{i} \frac{a h_{0} A}{(2 \pi)^{1 / 2}} \exp \left(-\mathrm{i} \omega_{0} t\right) \sin \theta_{0} \cos ^{2} \theta_{0} \sum_{ \pm}( \pm) \operatorname{sign} z_{ \pm} \\
& \times \int_{0}^{\infty} \kappa^{2} \exp \left(-\kappa^{2} a^{2} \cos ^{2} \theta_{0} / 2\right) \exp \left(-\beta \kappa^{3}\left|z_{ \pm}\right|\right) \exp \left( \pm \mathrm{i} \kappa x_{ \pm} \operatorname{sign} z_{ \pm}\right) \mathrm{d} \kappa
\end{aligned}
$$

Figure 13 applies the present theory to the contour maps in figures 2 and 4 of Peacock et al. (2008). The overall agreement with experiment is surprisingly good, given that the topography is nowhere as thin as the theory assumes it to be. This is confirmed in the far field by plotting in figures 14 and 15 the transverse profiles at cross sections $z_{+}=4 a$ and $12 a$, corresponding to figures 3 and 5 of Peacock et al. (2008), respectively: there is a tendency to overprediction of the wave amplitudes by the thin-topography approximation, and a shift of the experiment to the left for $\varepsilon=0.34$ in figure 14 compared with this approximation, but otherwise the shape of the profile is relatively well predicted. The theory of Balmforth et al. (2002) for finite topography seems to exhibit a similar shift in their figures $8(a, b)$, obtained in an inviscid fluid for similar criticality parameters $\varepsilon=0.4$ and 0.8 . As a rule, in these figures, each beam seems to be shifted to the side of the topography that it appears to emanate from, namely to the left for the beam pointing upward to the right, and to the right for the beam pointing upward to the left, though it is difficult to draw any definite conclusion based on such limited sample. 

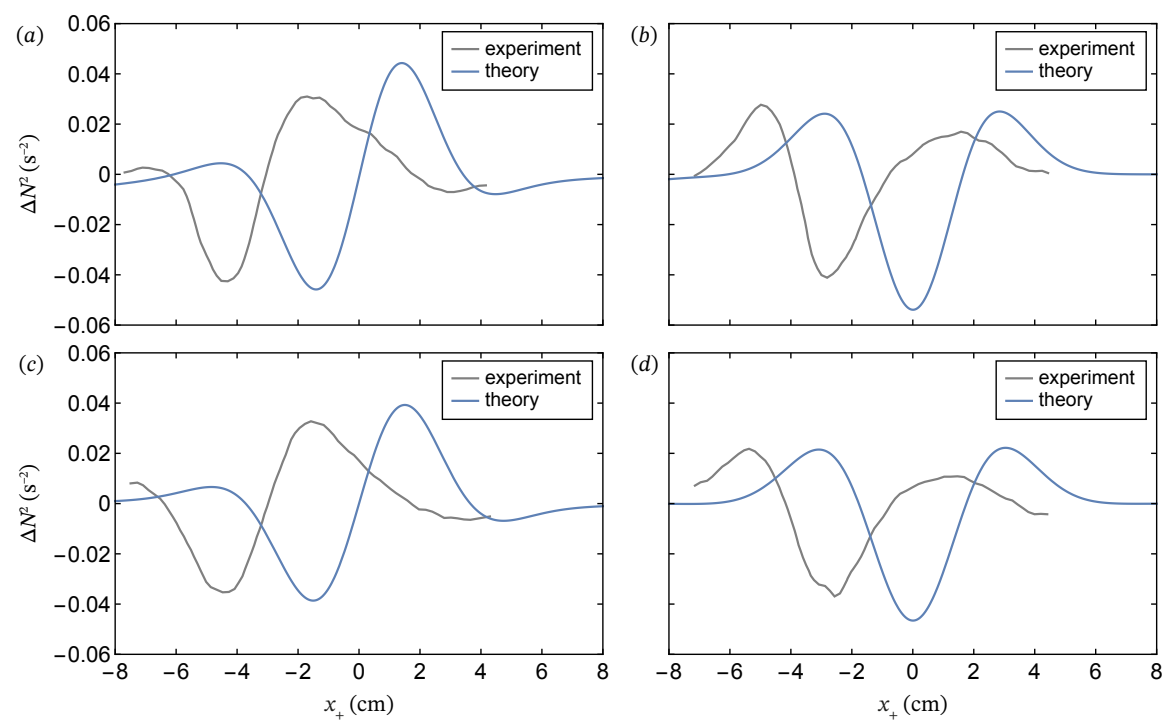

FIGURE 14. Transverse variations of $\Delta N^{2}$ at cross sections $(a, b) z_{+} / a=4$ and $(c, d) z_{+} / a=12$, at phases $(a, c) \phi=0$ and $(b, d) \phi=\pi / 2$, for the oscillations at relative frequency $\omega_{0} / N=0.80$ of the Gaussian bump in figure 3 of Peacock et al. (2008), with other parameters as in figure 13(a) above. The experimental data are plotted together with the present theory (8.50).
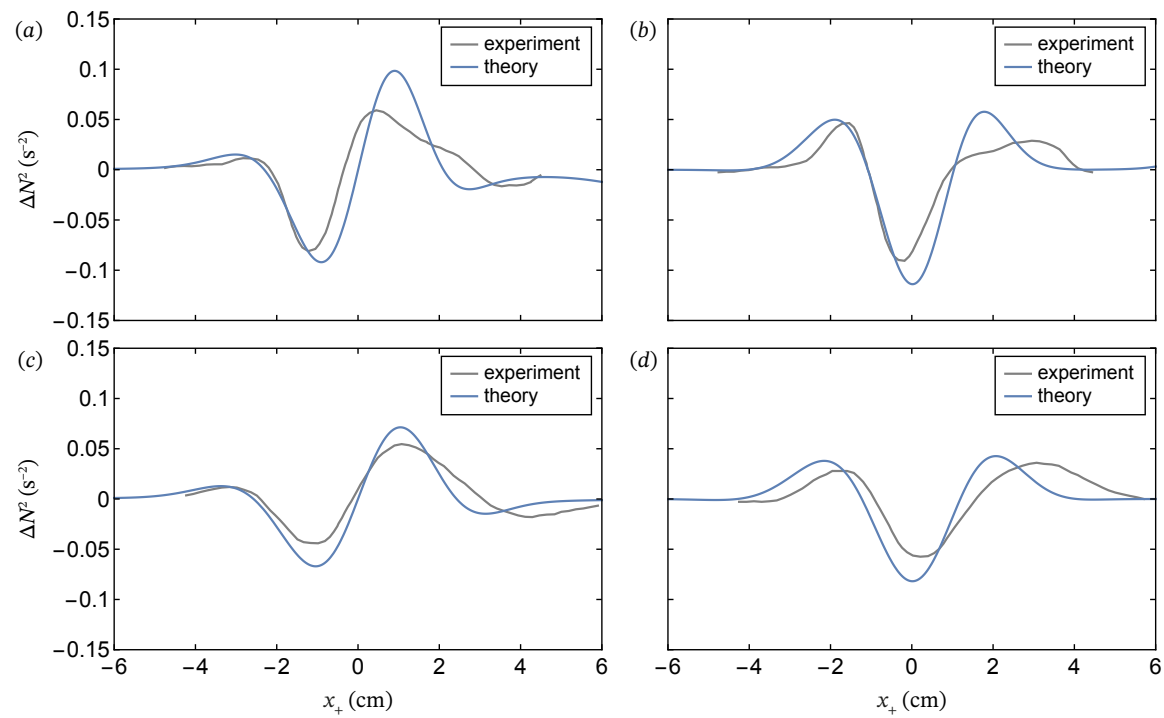

FIGURE 15. Same as figure 14, for the oscillations at relative frequency $\omega_{0} / N=0.48$ of the Gaussian bump in figure 5 of Peacock et al. (2008), with other parameters as in figure 13(b) above.

Close-ups of the near field are provided in figures 16 and 17. Owing to the absence of critical points, the wave variations are much smoother than for the supercritical sources in figures 6 and 10. The classical theory still yields segments of discontinuity along the lines $z_{ \pm}=0$, whose extension into the fluid is consistent with the above prediction $\left|x_{ \pm}\right| \lesssim 4 a \cos \theta_{0}$. Coming back to figures $13(a, b)$ and comparing them with experiment, the variations of the present theory are seen to differ from the experimental variations close to the topography, probably owing to the combined effects of the thin-topography 

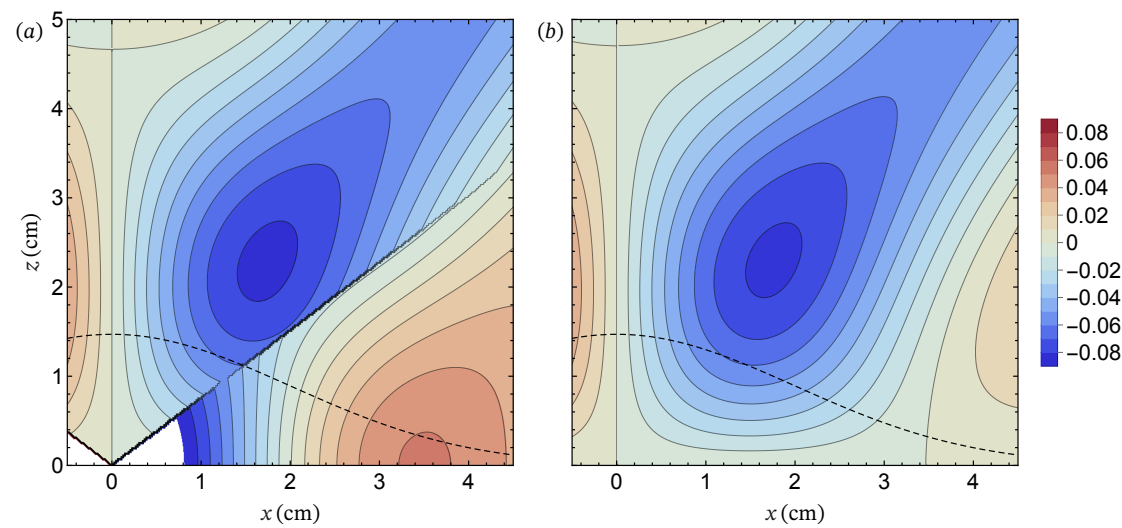

FIGURE 16. Near field for figure 13(a), using $(a)$ the classical theory $(8.51)$ and $(b)$ the present theory (8.50). The waves are calculated both inside and outside the bump, whose outline is shown dashed. The white areas correspond to off-scale values of the plotted quantity.
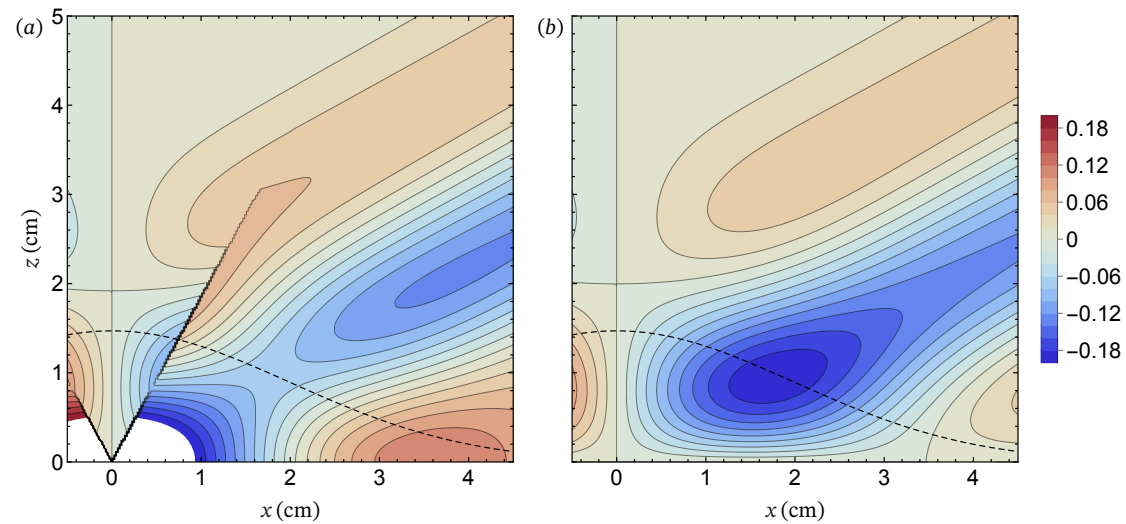

FIGURE 17. Near field for figure 13(b). The mode of representation is the same as in figure 16.

approximation and the free-slip boundary condition. Overall, comparing figures 9, 13(b) and 13(a), the rate of decrease of the wave amplitude with distance away from the forcing seems to become smaller as the criticality parameter $\varepsilon$ becomes smaller.

\section{Conclusion}

A new approach has been proposed for the generation of two-dimensional internal wave beams in fluids of small viscosity, extending to the near field the classical approach of Lighthill (1960, 1978, §4.10) for the far field. For this, the source of the waves has been assumed to be of compact support, a subclass of the rapidly decreasing sources considered by Lighthill. The waves have been derived by Fourier integration and application of the residue theorem in the wavenumber plane. Depending on the direction along which the theorem is applied, several expressions of the waves have been obtained: (6.1), most appropriate for a circular source; (4.26), most appropriate for an elliptic source, becoming (6.6) when the axes of the ellipse are horizontal and vertical; and (6.2), most appropriate for a source in the shape of an inclined strip, becoming (6.8) when the strip is horizontal and (6.9) when it is vertical. In each case, the indicated shape is the shape of the support domain used for the calculation of the waves. 
This support does not need to exactly match the shape of the source; the closer it does, the more accurate the expression of the waves, and the larger its domain of validity. For a given source, each expression of the waves has a specific domain of validity, all expressions becoming equivalent in the intersection of their domains. The equivalence is exact when the fluid is inviscid, and asymptotic, in the limit of large Stokes number, when the fluid is viscous.

These conclusions have been confirmed by application in $\S 8.1$ to the oscillations of a horizontal cylinder. When the cylinder is circular and (6.1) is used, the theory of Hurley \& Keady (1997) is recovered, which the experiments of Sutherland et al. $(1999,2000)$ and Zhang et al. (2007) have shown to apply everywhere in the fluid. When the cylinder is elliptic and (6.6) is used, the result is seen to satisfy the free-slip condition at the surface of the cylinder, hence to apply everywhere in the fluid, and to be consistent with the experiments of Sutherland \& Linden (2002). In both cases, viscosity arises only as an exponential attenuation factor in the integral expression of the waves. When the other expressions of the waves are used, associated with a support which does not match exactly the shape of the cylinder, viscosity arises also as an additional term inside the source spectrum. When this term, absent from the far-field theory of Lighthill $(1978, \S 4.10)$, is taken into account, every expression leads to correct prediction of the critical points at which critical wave rays are tangential to the cylinder.

This is especially important with a view to later extension of the analysis to include nonlinear effects and no-slip boundaries. For the former, the experimental investigations of Zhang et al. (2007), Ermanyuk, Flór \& Voisin (2011) and Shmakova, Ermanyuk \& Flór (2017), together with the numerical investigations of Tabaei, Akylas \& Lamb (2005) and Korobov \& Lamb (2008), have shown that nonlinear higher harmonics are generated for an oscillating body at the locations where the critical rays either intersect each other or are tangential to the body; for the latter, Kerswell (1995) and Le Dizès \& Le Bars (2017) have shown that the internal shear layers forming the wave beams arise from eruptions of the boundary layer at the critical points.

When the source reduces to a line, generating the waves (5.9) for an inclined line, (5.15) for a horizontal line and (5.19) for a vertical line, a boundary layer is produced in addition to the waves, given by (5.13), (5.16) and (5.20), respectively, with penetration depth

$$
\delta_{\mathrm{b}}=\left(\frac{2 \nu}{\omega_{0}}\right)^{1 / 2} \frac{\cos \theta_{0}}{\left|\cos \left(\theta_{0}+\varphi_{0}\right) \cos \left(\theta_{0}-\varphi_{0}\right)\right|^{1 / 2}},
$$

for a line inclined at the angle $\varphi_{0}$ to the horizontal generating waves propagating at the angle $\theta_{0}$ to the vertical, with $\nu$ the kinematic viscosity and $\omega_{0}$ the oscillation frequency. The other properties of the layer are, however, speculative, given the model of the forcing as a source term in the wave equation. The determination of this model for an actual forcing mechanism, typically the oscillations of a plate, requires the calculation of the boundary layer, which then predates the calculation of the waves. In three dimensions, the boundary layer has been obtained by Davis \& Llewellyn Smith (2010) and Le Dizès (2015) by solving the no-slip boundary-value problem for oscillating horizontal discs.

The three applications in $\S \S 8.2,8.3$ and 8.4 to a vertical knife edge, a vertical wave generator and a thin Gaussian bump, respectively, all involve line sources. For the knife edge the boundary layer is predicted to be of the same order as the waves and to yield singularities extending through the fluid at the level of the tip of the knife edge. This unphysical result illustrates the singularity of the low-viscosity limit $S t \rightarrow \infty$ for the boundary layer, where $S t$ is the Stokes number. As a rule, the present calculations for oscillating bodies assume free-slip boundaries. Accordingly, the viscous attenuation of 
the waves is accounted for but not the viscous boundary layer around the body. In this respect, as pointed out by an anonymous referee, the present study applies to the "near field' but not the 'near near-field'.

Quantitative validation of the study has been limited to the far field, where most of the available experimental measurements have been made. This is, unfortunately, also the region where the present theory and the classical theory of Lighthill $(1978, \S 4.10)$ and Hurley \& Keady (1997) become identical. In the near field, the validation has been limited to showing that the unphysical lines of singularities predicted by the classical theory are absent in the present theory, and that critical points are correctly accounted for. These points are visible to some extent in the experiments of Sutherland \& Linden (2002), but a quantitative study of the near field though simulations, if not experiments, remains to be done, which only will be able to provide independent verification of the theory.

When the shape of the oscillating body becomes more involved, the description of the wave field close to the body, where locally inward energy flux may be observed (Martin \& Llewellyn Smith 2012a), cannot be achieved via a Fourier-based method. The Green's function method provides a convenient alternative, in which the response to a point source $\delta(x) \delta(z)$ is represented by the Green's function $G(x, z)$ and the waves produced by an arbitrary source $f(x, z)$ follow from its convolution with the Green's function,

$$
\psi(x, z)=\iint f\left(x^{\prime}, z^{\prime}\right) G\left(x-x^{\prime}, z-z^{\prime}\right) \mathrm{d} x^{\prime} \mathrm{d} z^{\prime} .
$$

Voisin (2003) and Martin \& Llewellyn Smith (2012a) have applied this method to the determination of the far field for a generic three-dimensional source and an oscillating sphere, respectively, and Bühler \& Muller (2007) have evaluated the convolution integral numerically for a variety of thin topographies. The derivation of the two-dimensional Green's function is presented in Appendix B for both inviscid and viscous fluids.

The next step in this study is its adaptation to three dimensions. The expression (2.7) of three-dimensional waves in Voisin et al. (2011), based on an extrapolation from Voisin (2003), is of the same type as the present (6.9), but misses the restriction $|z|>b$ on its validity and the viscous correction inside the spectrum of the forcing. As a result, its application (2.16) to an oscillating sphere, though in agreement with the measurements of Flynn, Onu \& Sutherland (2003), King, Zhang \& Swinney (2009), Voisin et al. (2011) and Ghaemsaidi \& Peacock (2013), performed mostly in the far field, yields spurious singularities in the equatorial plane of the sphere in the near field, visible in figures 6 and 7 of Voisin et al. (2011). No such singularity has been found in the experiments of Flynn et al. (2003), Sutherland, Flynn \& Onu (2003) and King et al. (2009), or the numerical simulations of King et al. (2009), Bigot et al. (2014) and Le Dizès \& Le Bars (2017). The interested reader may also check that, in the inviscid case, the expression (2.16) of the waves in Voisin et al. (2011) does not satisfy the free-slip boundary condition at the sphere in between the critical latitudes, that is for $|z|<a \sin \theta_{0}$.

An added complexity in three dimensions is the existence of a complex continuation to the real wavenumber surface. To illustrate this, we write the dispersion relation in Cartesian components $(k, l, m)$ as

$$
\left(k^{2}+l^{2}\right) \sin ^{2} \theta_{0}=m^{2} \cos ^{2} \theta_{0} .
$$

In two dimensions, $l=0$ and for each real $k$ this equation has two real solutions $m=$ $\pm k \tan \theta_{0}$, yielding the wavenumber surface in the shape of a St. Andrew's Cross shown in figure 1. In three dimensions, if $k$ and $l$ are real and integration is performed over $m$, as in Voisin (2003), the two solutions $m= \pm\left(k^{2}+l^{2}\right)^{1 / 2}$ are real and define a conical 
wavenumber surface. If $k$ and $m$ are real and integration is performed over $l$, however, the two solutions are $l= \pm\left(m^{2} \cot ^{2} \theta_{0}-k^{2}\right)^{1 / 2}$ : if $|m|>|k| \tan \theta_{0}$ they belong to the intersection of the conical surface with the plane of constant $l$, but if $|m|<|k| \tan \theta_{0}$ they become imaginary and migrate outside this surface; in either case they are valid solutions which need to be taken into account, especially in the near field. This possibility, foreseen by Lighthill (1990) for general waves, does not exist in two dimensions. Work to adapt the analysis to this situation is under way.

Finally, it must be pointed out that other diffusive phenomena are present in real fluids in addition to viscosity, affecting temperature and concentration. They have been ignored to keep the analysis tractable, but may play a role in applications. As a rule, the diffusivities add up to viscosity in the expression of the attenuation coefficient of the waves, and give rise to new boundary layers, one per diffusivity. Their effect on the dispersion relation has been investigated by Kistovich \& Chashechkin (2007) and Chashechkin (2018), the latter presenting experimental examples of the flow structure near oscillating discs and spheres. Molecular diffusivity is a common occurrence in stratified fluids at laboratory scale, and has been taken into account by Kistovich \& Chashechkin (1995) for the reflection of a two-dimensional wave beam at an inclined plane, Bardakov et al. (2007) and Davis \& Llewellyn Smith (2010) for the emission of waves by an oscillating disc and Vasil'ev \& Chashechkin (2012) for the emission by an oscillating plate. In astrophysical conditions magnetic and thermal diffusivities may also play a role, investigated for oscillating discs by Tilgner (2000) and Le Dizès (2015), respectively.

\section{Acknowledgements}

The author would like to thank L. Maas, E. Ermanyuk, N. Shmakova, S. Le Dizès, T. Dauxois, M. Mercier, P.-P. Cortet, S. Dalziel, B. Sutherland and J. Sommeria (in rough chronological order) for interesting conversations and correspondence. The referees are thanked for insightful suggestions which led to substantial improvement of the paper.

\section{Declaration of interests}

The author reports no conflict of interest.

\section{Appendix A. Higher-order sources}

The bounds (2.20) and (4.13) are a lesser form of a family of theorems, known as Paley-Wiener theorems after their introduction by Paley \& Wiener $(1934, \S 6)$ for squareintegrable functions, relating the decay properties of a function to the behaviour of its Fourier transform in the complex plane. For a function $f(x)$ of compact support of radius $a$, such that $f(x)=0$ for $|x|>a$, the theorem states that the transform

$$
f(k)=\int_{-a}^{a} f(x) \exp (-\mathrm{i} k x) \mathrm{d} x
$$

is an analytic function of the complex variable $k$, integrable along the real axis and growing exponentially along the imaginary axis; by the latter it is meant that there exists a positive constant $C$ such that, for all complex $k$,

$$
|f(k)|<C \exp (a|\operatorname{Im} k|) \text {. }
$$


The theorem also states that the reciprocal is true: any function having these properties is the Fourier transform of an integrable function of compact support of radius $a$. When the original function is further smooth, namely differentiable to any order, then each derivative $f^{(n)}(x)$, of transform (ik) $f(k)$, admits a bound of the form (A 2); as a result $f(k)$ is rapidly decreasing along the real axis, namely decreasing faster than any inverse power of $k$, since for any non-negative integer $n$ there exists a positive constant $C_{n}$ such that, for all complex $k$,

$$
|f(k)|<C_{n}(1+|k| a)^{-n} \exp (a|\operatorname{Im} k|) .
$$

Schwartz extended this theorem to distributions of order $n$, such as the $n$-th derivative of the Dirac delta function. According to the extension (Hörmander 1990, §7.3), the Fourier transform $f(k)$ of a distribution $f(x)$ of order $n$ and compact support of radius $a$ is an analytic function of the complex variable $k$, with slow polynomial growth along the real axis and exponential growth along the imaginary axis; specifically, there exists a positive constant $C$ such that, for all complex $k$,

$$
|f(k)|<C(1+|k| a)^{n} \exp (a|\operatorname{Im} k|) .
$$

Again, the reciprocal is true: any function having these properties is the Fourier transform of a distribution of order $n$ and compact support of radius $a$.

Here, given (3.5), where the rational fraction multiplying the spectrum varies as the inverse wavenumber, this means that the analysis of $\$ \S 4$ and 5 only applies to source functions that are either proper functions or distributions of order $n=0$ (as in $\S 8.1$ ). For higher $n=1,2, \ldots$ (as in $\S 8.2$ ), the contribution of the semi-circle at infinity does not vanish and must be evaluated.

We present a purely heuristic derivation for the line source (5.3). For simplicity we set $\nu=0$. The integrand in (4.3) behaves asymptotically for large $\left|m_{0}\right|$ as

$$
-\mathrm{i}^{n}\left[\boldsymbol{e}_{z_{0}}+\boldsymbol{e}_{x_{0}} \frac{\sin \varphi_{0} \cos \varphi_{0}}{\cos \left(\theta_{0}+\varphi_{0}\right) \cos \left(\theta_{0}-\varphi_{0}\right)}\right] g\left(k_{0}\right) m_{0}^{n-1} \exp \left[\mathrm{i}\left(k_{0} x_{0}+m_{0} z_{0}\right)\right] \text {. }
$$

We use this estimate along the semi-circle at infinity, close the semi-circle by a straight line along the real axis, use along this line the inverse transform

$$
\int_{-\infty}^{\infty} k^{n} \exp (\mathrm{i} k x) \mathrm{d} k=2 \pi(-\mathrm{i})^{n} \delta^{(n)}(x),
$$

taken from table 4 of Voisin (2003), and apply Cauchy's theorem. The result is a new contribution to the velocity,

$$
\boldsymbol{u}_{\infty}=\left[\boldsymbol{e}_{z_{0}}+\boldsymbol{e}_{x_{0}} \frac{\sin \varphi_{0} \cos \varphi_{0}}{\cos \left(\theta_{0}+\varphi_{0}\right) \cos \left(\theta_{0}-\varphi_{0}\right)}\right] \exp \left(-\mathrm{i} \omega_{0} t\right) g\left(x_{0}\right) \delta^{(n-1)}\left(z_{0}\right),
$$

in the form of a singularity of order $n-1$ at the source. Such singularity is an artifact of the source model and has been ignored throughout.

\section{Appendix B. Green's function}

The Green's function is defined as the solution of the wave equation (2.1) or (2.23) for unit point forcing $f(x, z)=\delta(x) \delta(z)$. The flatness of the spectrum $f(k, m)=1$ leaves the wavenumber arbitrary, allowing $\left|k_{ \pm}\right|$to become infinitely large and preventing the poles (2.15) and (2.26) from remaining small, however small $\epsilon / N$ and $\beta \kappa^{2}$ can be. The derivations in $\S 2$ are invalidated and alternative derivations are necessary, presented briefly in this appendix. The time dependence as $\exp \left(-i \omega_{0} t\right)$ is implicit throughout. 


\section{B.1. Inviscid case}

The inviscid Green's function satisfies

$$
\left[\left(\omega_{0}^{2}-N^{2}\right) \frac{\partial^{2}}{\partial x^{2}}+\omega_{0}^{2} \frac{\partial^{2}}{\partial z^{2}}\right] G=-\delta(x) \delta(z),
$$

an equation solved by the method of Bryan (1889) and Hurley (1972). For $\omega_{0}>N$ the equation is elliptic. Stretching the coordinates according to

$$
x_{\star}=\frac{\omega_{0}}{N} x, \quad z_{\star}=\frac{\left(\omega_{0}^{2}-N^{2}\right)^{1 / 2}}{N} z,
$$

transforms it into a Poisson equation, of known Green's function $-1 /(4 \pi) \ln \left(x_{\star}^{2}+z_{\star}^{2}\right)$. The solution is continued analytically onto the upper half of the complex $\omega_{0}$-plane, so as to ensure causality. For real $\omega_{0}$ this gives

$$
G=-\frac{\ln \left[\left(\omega_{0}+\mathrm{i} 0\right)^{2} r^{2}-N^{2} z^{2}\right]}{4 \pi \omega_{0}\left[\left(\omega_{0}+\mathrm{i} 0\right)^{2}-N^{2}\right]^{1 / 2}},
$$

where the addition of an infinitesimal positive imaginary part to $\omega_{0}$ is consistent with Lighthill's radiation condition.

For $0<\omega_{0}<N$ we may write, up to an insignificant constant,

$$
G=\mathrm{i} \frac{\ln \left(x_{+} x_{-}+\mathrm{i} 0\right)}{4 \pi N^{2} \sin \theta_{0} \cos \theta_{0}},
$$

or equivalently in decomposed form,

$$
G=\frac{\mathrm{i}}{4 \pi N^{2} \sin \theta_{0} \cos \theta_{0}}\left[\ln \left(x_{+}+\mathrm{i} 0 \operatorname{sign} x_{-}\right)+\ln \left(x_{-}+\mathrm{i} 0 \operatorname{sign} x_{+}\right)\right] .
$$

The fluid velocity follows by differentiation according to

$$
\boldsymbol{u}_{G}=N^{2}\left(\boldsymbol{e}_{x} \sin ^{2} \theta_{0} \frac{\partial}{\partial x}-\boldsymbol{e}_{z} \cos ^{2} \theta_{0} \frac{\partial}{\partial z}\right) G,
$$

yielding

$$
\boldsymbol{u}_{G}=\mathrm{i} \frac{\sin \theta_{0} \cos \theta_{0}}{2 \pi} \frac{\boldsymbol{x}}{x_{+} x_{-}+\mathrm{i} 0},
$$

or in decomposed form,

$$
\boldsymbol{u}_{G}=\frac{\mathrm{i}}{4 \pi}\left(\frac{\boldsymbol{e}_{z_{+}}}{x_{+}+\mathrm{i} 0 \operatorname{sign} x_{-}}-\frac{\boldsymbol{e}_{z_{-}}}{x_{-}+\mathrm{i} 0 \operatorname{sign} x_{+}}\right) .
$$

The phase variations are put forward by writing

$$
\ln (x \pm \mathrm{i} 0)=\ln |x| \pm \mathrm{i} \pi H(-x), \quad \frac{1}{x \pm \mathrm{i} 0}=\operatorname{pv}\left(\frac{1}{x}\right) \mp \mathrm{i} \pi \delta(x),
$$

with pv the principal value. These results have first been obtained by Hurley (1969), considering the Boussinesq limit of the non-Boussinesq Green's function, and Bühler \& Muller (2007).

As a verification, we note that the logarithm in (B 4) has the determination

$$
\ln \left|x_{+} x_{-}\right|+\mathrm{i} \frac{\pi}{2}\left(1-\operatorname{sign} x_{+} \operatorname{sign} x_{-}\right)
$$


while the analysis of $\S 2.1$, using the inverse transform

$$
\int_{0}^{\infty} \exp (\mathrm{i} k x) \frac{\mathrm{d} k}{k}=\mathrm{i} \frac{\pi}{2}-\ln (x+\mathrm{i} 0),
$$

taken from table 5 of Voisin (2003), would give instead

$$
\ln \left(x_{+}+\mathrm{i} 0 \operatorname{sign} z_{+}\right)-\mathrm{i} \frac{\pi}{2} \operatorname{sign} z_{+}+\ln \left(x_{-}-\mathrm{i} 0 \operatorname{sign} z_{-}\right)+\mathrm{i} \frac{\pi}{2} \operatorname{sign} z_{-},
$$

with determination

$$
\ln \left|x_{+} x_{-}\right|+\mathrm{i} \frac{\pi}{2}\left(\operatorname{sign} x_{-} \operatorname{sign} z_{-}-\operatorname{sign} x_{+} \operatorname{sign} z_{+}\right),
$$

yielding incorrect phase jumps across the lines $x_{ \pm}=0$ and adding spurious phase jumps across the lines $z_{ \pm}=0$.

\section{B.2. Viscous case}

The viscous Green's function is given by (2.24) as the inverse transform

$$
G=\frac{1}{4 \pi^{2}} \iint \frac{\exp [\mathrm{i}(k x+m z)]}{\omega_{0}^{2} \kappa^{2}-N^{2} k^{2}+\mathrm{i} \omega_{0} \nu \kappa^{4}} \mathrm{~d} k \mathrm{~d} m .
$$

We evaluate it by applying the residue theorem to integration over $m$. Jordan's lemma selects the two poles $m=m_{1,2}$ sign $z$, where

$$
m_{1,2}^{2}=-k^{2}+\mathrm{i} \frac{\omega_{0}}{2 \nu}[1 \mp D(k)], \quad \operatorname{Im} m_{1,2}>0,
$$

and the square root

$$
D(k)=\left(1+\mathrm{i} \frac{4 \nu k^{2}}{\omega_{0} \cos ^{2} \theta_{0}}\right)^{1 / 2}
$$

is taken in the first quadrant. This gives

$$
G=\frac{\mathrm{i}}{4 \pi N^{2} \cos ^{2} \theta_{0}} \int_{-\infty}^{\infty} \frac{\exp (\mathrm{i} k x)}{D(k)}\left[\frac{\exp \left(\mathrm{i} m_{1}|z|\right)}{m_{1}}-\frac{\exp \left(\mathrm{i} m_{2}|z|\right)}{m_{2}}\right] \mathrm{d} k .
$$

The fluid velocity follows by differentiation according to

$$
\boldsymbol{u}_{G}=N^{2}\left[\boldsymbol{e}_{x}\left(\sin ^{2} \theta_{0}+\mathrm{i} \cos ^{2} \theta_{0} \frac{\nu \nabla^{2}}{\omega_{0}}\right) \frac{\partial}{\partial x}-\boldsymbol{e}_{z}\left(\cos ^{2} \theta_{0}-\mathrm{i} \cos ^{2} \theta_{0} \frac{\nu \nabla^{2}}{\omega_{0}}\right) \frac{\partial}{\partial z}\right] G,
$$

yielding

$$
\begin{array}{r}
u_{G}=\frac{1}{8 \pi} \int_{-\infty}^{\infty} \exp (\mathrm{i} k x)\left\{\left[1-\frac{1+2 \tan ^{2} \theta_{0}}{D(k)}\right] \frac{k}{m_{1}} \exp \left(\mathrm{i} m_{1}|z|\right)\right. \\
\left.+\left[1+\frac{1+2 \tan ^{2} \theta_{0}}{D(k)}\right] \frac{k}{m_{2}} \exp \left(\mathrm{i} m_{2}|z|\right)\right\} \mathrm{d} k \\
w_{G}=\frac{\operatorname{sign} z}{8 \pi} \int_{-\infty}^{\infty} \exp (\mathrm{i} k x)\left\{\left[1+\frac{1}{D(k)}\right] \exp \left(\mathrm{i}_{1}|z|\right)\right. \\
\left.+\left[1-\frac{1}{D(k)}\right] \exp \left(\mathrm{i} m_{2}|z|\right)\right\} \mathrm{d} k
\end{array}
$$


These results have first been obtained by Ramachandra Rao \& Balan (1977). Their derivation closely follows those by Chashechkin, Vasil'ev \& Bardakov (2004) and Davis \& Llewellyn Smith (2010) for the waves generated by an oscillating disc.

In the inviscid limit $\nu \rightarrow 0$ we have

$$
m_{1} \sim-|k| \tan \theta_{0}, \quad m_{2} \sim\left(\frac{\omega_{0}}{2 \nu}\right)^{1 / 2}(1+\mathrm{i}),
$$

implying that the term of vertical wavenumber $m_{2}$ vanishes and the term of vertical wavenumber $m_{1}$ reduces to the inviscid Green's function.

\section{REFERENCES}

Appleby, J. C. \& CRIGHTON, D. G. 1986 Non-Boussinesq effects in the diffraction of internal waves from an oscillating cylinder. Q. J. Mech. Appl. Math. 39, 209-231.

APPLEBY, J. C. \& CRIGHTON, D. G. 1987 Internal gravity waves generated by oscillations of a sphere. J. Fluid Mech. 183, 439-450.

BALMFORTH, N. J., IERLEY, G. R. \& YOUNG, W. R. 2002 Tidal conversion by subcritical topography. J. Phys. Oceanogr. 32, 2900-2914.

BALMFORTH, N. J. \& PEACOCK, T. 2009 Tidal conversion by supercritical topography. J. Phys. Oceanogr. 39, 1965-1974.

BARDAKov, R. N., VASIL'EV, A. YU. \& CHASHECHKIN, YU. D. 2007 Calculation and measurement of conical beams of three-dimensional periodic internal waves excited by a vertically oscillating piston. Fluid Dyn. 42, 612-626.

Beckebanze, F., Brouzet, C., Sibgatullin, I. N. \& MAAS, L. R. M. 2018 Damping of quasi-twodimensional internal wave attractors by rigid-wall friction. J. Fluid Mech. 841, 614-635.

BECKEBANZE, F., RAJA, K. J. \& MAAS, L. R. M. 2019 Mean flow generation by three-dimensional nonlinear internal wave beams. J. Fluid Mech. 864, 303-326.

BELL, T. H. 1975a Lee waves in stratified flows with simple harmonic time dependence. J. Fluid Mech. 67, 705-722.

BELL, T. H. 1975b Topographically generated internal waves in the open ocean. J. Geophys. Res. 80, 320-327.

Bigot, B., BonometTi, T., LACAZE, L. \& Thual, O. 2014 A simple immersed-boundary method for solid-fluid interaction in constant- and stratified-density flows. Comput. Fluids 97, 126-142.

BOURY, S., PEACOCK, T. \& OdIER, P. 2019 Excitation and resonant enhancement of axisymmetric internal wave modes. Phys. Rev. Fluids 4, 034802.

BRUnet, M., DAuXoIS, T. \& CORTET, P.-P. 2019 Linear and nonlinear regimes of an inertial wave attractor. Phys. Rev. Fluids 4, 034801.

BRYAN, G. H. 1889 The waves on a rotating liquid spheroid of finite ellipticity. Phil. Trans. R. Soc. A 180, 187-219.

BÜHLER, O. \& MULLER, C. J. 2007 Instability and focusing of internal tides in the deep ocean. J. Fluid Mech. 588, 1-28.

ChASHEChKin, YU. D. 2018 Singular perturbed components of flows - linear precursors of shock waves. Math. Model. Nat. Phenom. 13, 17.

ChashechKin, YU. D. \& Kistovich, YU. V. 1997 Generation of monochromatic internal waves: an exact solution and the force-source model. Phys. Dokl. 42, 377-380.

Chashechkin, Yu. D., VAsil'EV, A. YU. \& BARdakov, R. N. 2004 Fine structure of beams of a threedimensional periodic internal wave. Dokl. Earth Sci. 397A, 816-819.

Cox, C. \& SANDSTROM, H. 1962 Coupling of internal and surface waves in water of variable depth. J. Oceanogr. Soc. Jpn 20th Anniversary Volume, 499-513.

DAlziel, S. B., HugheS, G. O. \& SUTHERLAND, B. R. 2000 Whole-field density measurements by 'synthetic schlieren'. Exp. Fluids 28, 322-335.

DAUXois, T., Joubaud, S., OdieR, P. \& VenAILLE, A. 2018 Instabilities of internal gravity wave beams. Annu. Rev. Fluid Mech. 50, 131-156.

DAVIS, A. M. J. 2012 Generation of internal waves from rest: extended use of complex coordinates, for a sphere but not a disk. J. Fluid Mech. 703, 374-390. 
DAVIS, A. M. J. \& LLEwELLYN SMITH, S. G. 2010 Tangential oscillations of a circular disk in a viscous stratified fluid. J. Fluid Mech. 656, 342-359.

Dobra, T. E., LAWRIE, A. G. W. \& DALZIEL, S. B. 2019 The magic carpet: an arbitrary spectrum wave maker for internal waves. Exp. Fluids 60, 172.

Dossmann, Y., Bourget, B., Brouzet, C., Dauxois, T., Joubaud, S. \& Odier, P. 2016 Mixing by internal waves quantified using combined PIV/PLIF technique. Exp. Fluids 57, 132.

Dossmann, Y., Pollet, F., OdieR, P. \& DAuxois, T. 2017 Mixing and formation of layers by internal wave forcing. J. Geophys. Res. Oceans 122, 9906-9917.

ECHEVERRI, P. \& PEACOCK, T. 2010 Internal tide generation by arbitrary two-dimensional topography. J. Fluid Mech. 659, 247-266.

ECHeVERri, P., Yokossi, T., BALMforth, N. J. \& PEACock, T. 2011 Tidally generated internal-wave attractors between double ridges. J. Fluid Mech. 669, 354-374.

ERMANYUK, E. V., FLÓR, J.-B. \& VOISIN, B. 2011 Spatial structure of first and higher harmonic internal waves from a horizontally oscillating sphere. J. Fluid Mech. 671, 364-383.

ERMANYUK, E. V. \& GAVRILOV, N. V. 2005 Duration of transient processes in the formation of internalwave beams. Dokl. Phys. 50, 548-550.

FALAHAT, S., NyCANDER, J., RoQUET, F. \& ZARROUG, M. 2014 Global calculation of tidal energy conversion into vertical normal modes. J. Phys. Oceanogr. 44, 3225-3244.

FLYNN, M. R., ONU, K. \& SUTHERLAND, B. R. 2003 Internal wave excitation by a vertically oscillating sphere. J. Fluid Mech. 494, 65-93.

GABOv, S. A. 1985 The solution of a problem of stratified fluid dynamics and its stabilization as $t \rightarrow \infty$. USSR Comput. Math. Math. Phys. 25 (3), 47-55.

GABOV, S. A. \& KRUTITSKII, P. A. 1987 On the non-stationary Larsen problem. USSR Comput. Math. Math. Phys. 27 (4), 148-154.

Gabov, S. A. \& Pletner, Yu. D. 1985 An initial-boundary value problem for the gravitationalgyroscopic wave equation. USSR Comput. Math. Math. Phys. 25 (6), 64-68.

GABOV, S. A. \& PLETNER, YU. D. 1988 The problem of the oscillations of a flat disc in a stratified liquid. USSR Comput. Math. Math. Phys. 28 (1), 41-47.

GABOV, S. A. \& SHEVTSOV, P. V. 1983 Basic boundary value problems for the equation of oscillations of a stratified fluid. Sov. Math. Dokl. 27, 238-241.

GABOv, S. A. \& SheVTSOV, P. V. 1984 On a differential equation of the type of Sobolev's equation. Sov. Math. Dokl. 29, 411-414.

GARRETT, C. \& KunZE, E. 2007 Internal tide generation in the deep ocean. Annu. Rev. Fluid Mech. 39, 57-87.

GHAEMSAIDI, S. J. \& PEACOCK, T. 2013 3D Stereoscopic PIV visualization of the axisymmetric conical internal wave field generated by an oscillating sphere. Exp. Fluids 54, 1454.

GÖRTLER, H. 1943 Über eine Schwingungserscheinung in Flüssigkeiten mit stabiler Dichteschichtung. Z. Angew. Math. Mech. 23, 65-71.

GöRTLER, H. 1944 Einige Bemerkungen über Strömungen in rotierenden Flüssigkeiten. Z. Angew. Math. Mech. 24, 210-214.

Gostiaux, L., Didelle, H., Mercier, S. \& Dauxois, T. 2007 A novel internal waves generator. Exp. Fluids 42, 123-130.

HENDERSHOTT, M. C. 1969 Impulsively started oscillations in a rotating stratified fluid. J. Fluid Mech. 36, 513-527.

HöRMANDER, L. 1990 The Analysis of Linear Partial Differential Operators I, 2nd edn. Springer.

HURLEY, D. G. 1969 The emission of internal waves by vibrating cylinders. J. Fluid Mech. 36, 657-672.

HURLEY, D. G. 1972 A general method for solving steady-state internal gravity wave problems. J. Fluid Mech. 56, 721-740.

HURLEY, D. G. 1997 The generation of internal waves by vibrating elliptic cylinders. Part 1. Inviscid solution. J. Fluid Mech. 351, 105-118.

HURLEY, D. G. \& HoOD, M. J. 2001 The generation of internal waves by vibrating elliptic cylinders. Part 3. Angular oscillations and comparison of theory with recent experimental observations. $J$. Fluid Mech. 433, 61-75.

HURLEY, D. G. \& KEADY, G. 1997 The generation of internal waves by vibrating elliptic cylinders. Part 2. Approximate viscous solution. J. Fluid Mech. 351, 119-138.

KAPITONOV, B. V. 1980 Potential theory for the equation of small oscillations of a rotating fluid. Math. USSR Sb. 37, 559-579. 
KATAOKA, T. \& AKYLAS, T. R. 2015 On three-dimensional internal gravity wave beams and induced large-scale mean flows. J. Fluid Mech. 769, 621-634.

KERSWELL, R. R. 1995 On the internal shear layers spawned by the critical regions in oscillatory Ekman boundary layers. J. Fluid Mech. 98, 311-325.

King, B., ZHANG, H. P. \& Swinney, H. L. 2009 Tidal flow over three-dimensional topography in a stratified fluid. Phys. Fluids 21, 116601.

Kistovich, A. V. \& CHASHechKIN, YU. D. 2007 Regular and singular components of periodic flows in the fluid interior. J. Appl. Maths Mech. 71, 762-771.

Kistovich, YU. V. \& CHASHECHKIN, YU. D. 1994 Reflection of packets of internal waves from a rigid plane in a viscous fluid. Izv. Atmos. Ocean. Phys. 30, 718-724.

Kistovich, YU. V. \& CHASHECHKIN, YU. D. 1995 The reflection of beams of internal gravity waves at a flat rigid surface. J. Appl. Math. Mech. 59, 579-585.

Kistovich, YU. V. \& CHASHECHKIN, YU. D. 1999a Generation of monochromatic internal waves in a viscous fluid. J. Appl. Mech. Tech. Phys. 40, 1020-1028.

Kistovich, YU. V. \& ChAshechKin, YU. D. $1999 b$ An exact solution of a linearized problem of the radiation of monochromatic internal waves in a viscous fluid. J. Appl. Math. Mech. 63, 587-594.

Korobov, A. S. \& LAMB, K. G. 2008 Interharmonics in internal gravity waves generated by tidetopography interaction. J. Fluid Mech. 611, 61-95.

KrishNA, D. V. \& SARMA, L. V. 1969 Motion of an axisymmetric body in a rotating stratified fluid confined between two parallel planes. J. Fluid Mech. 38, 833-842.

LAI, R. Y. S. \& LEE, C.-M. 1981 Added mass of a spheroid oscillating in a linearly stratified fluid. Int. J. Engng Sci. 19, 1411-1420.

LE DizÈs, S. 2015 Wave field and zonal flow of a librating disk. J. Fluid Mech. 782, 178-208.

LE Dizès, S. \& LE BARS, M. 2017 Internal shear layers from librating objects. J. Fluid Mech. 826, 653-675.

LighthiLL, M. J. 1958 An Introduction to Fourier Analysis and Generalised Functions. Cambridge University Press.

LighthiLL, M. J. 1960 Studies on magneto-hydrodynamic waves and other anisotropic wave motions. Phil. Trans. R. Soc. A 252, 397-430.

LighthiLL, J. 1978 Waves in Fluids. Cambridge University Press.

Lighthill, J. 1990 Emendations to a proof in the general three-dimensional theory of oscillating sources of waves. Proc. R. Soc. A 427, 31-42.

LLEWEllyn SMith, S. G. \& YounG, W. R. 2002 Conversion of the barotropic tide. J. Phys. Oceanogr. 32, 1554-1566.

Llewellyn SMith, S. G. \& YounG, W. R. 2003 Tidal conversion at a very steep ridge. J. Fluid Mech. 495, 175-191.

MAChicoANe, N., CORTET, P.-P., VoIsin, B. \& Moisy, F. 2015 Influence of the multipole order of the source on the decay of an inertial wave beam in a rotating fluid. Phys. Fluids 27, 066602.

MARTIN, P. A. \& LLEWELLYN SMITH, S. G. 2011 Generation of internal gravity waves by an oscillating horizontal disc. Proc. R. Soc. A 467, 3406-3423.

MARTIN, P. A. \& LLEWELLYN SMITH, S. G. $2012 a$ Internal gravity waves, boundary integral equations and radiation conditions. Wave Motion 49, 427-444.

MARTIN, P. A. \& LLEWELLYN SMITH, S. G. $2012 b$ Generation of internal gravity waves by an oscillating horizontal elliptical plate. SIAM J. Appl. Math. 72, 725-739.

MAUreR, P., GHAEMSAId, S. J., Joubaud, S., PEACOCK, T. \& OdIER, P. 2017 An axisymmetric inertiagravity wave generator. Exp. Fluids 58, 143.

Melet, A., Nikurashin, M., Muller, C., Falahat, S., Nycander, J., Timko, P. G., Arbic, B. K. \& GoFF, J. A. 2013 Internal tide generation by abyssal hills using analytical theory. J. Geophys. Res. Oceans 118, 6303-6318.

Mercier, M. J., Martinand, D., Mathur, M., Gostiaux, L., Peacock, T. \& Dauxois, T. 2010 New wave generation. J. Fluid Mech. 657, 308-334.

MooRE, D. W. \& SAFFMAN, P. G. 1969 The structure of free vertical shear layers in a rotating fluid and the motion produced by a slowly rising body. Phil. Trans. R. Soc. A 264, 597-634.

MOWBRAY, D. E. \& RARITY, B. S. H. 1967 A theoretical and experimental investigation of the phase configuration of internal waves of small amplitude in a density stratified liquid. J. Fluid Mech. 28, 1-16. 
Musgrave, R. C., Pinkel, R., MacKinnon, J. A., Mazloff, M. R. \& Young, W. R. 2016 Stratified tidal flow over a tall ridge above and below the turning latitude. J. Fluid Mech. 793, 933-957.

NYCANDER, J. 2005 Generation of internal waves in the deep ocean by tides. J. Geophys. Res. 110, C10028.

NYCANDER, J. 2006 Tidal generation of internal waves from a periodic array of steep ridges. J. Fluid Mech. 567, 415-432.

OGILVIE, G. I. 2005 Wave attractors and the asymptotic dissipation rate of tidal disturbances. J. Fluid Mech. 543, 19-44.

OSER, H. 1957 Erzwungene Schwingungen in rotierenden Flüssigkeiten. Arch. Ration. Mech. Anal. 1, 81-96.

OSER, H. 1958 Experimentelle Untersuchung über harmonische Schwingungen in rotierenden Flüssigkeiten. Z. Angew. Math. Mech. 38, 386-391.

PALEY, R. E. A. C. \& WIENER, N. 1934 Fourier Transforms in the Complex Domain. American Mathematical Society.

PEACOCK, T., ECHEVERRI, P. \& BALMFORTH, N. J. 2008 An experimental investigation of internal tide generation by two-dimensional topography. J. Phys. Oceanogr. 38, 235-242.

PÉTRÉLIS, F., LleWELlYN SMITH, S. \& YouNG, W. R. 2006 Tidal conversion at a submarine ridge. J. Phys. Oceanogr. 36, 1053-1071.

RAMACHANDRA RAO, A. \& BALAN, K. C. 1977 Effect of viscosity on internal waves from a source in a wall. Proc. Indian Acad. Sci. A 85, 351-366.

RENAUd, A. \& VenAILle, A. 2019 Boundary streaming by internal waves. J. Fluid Mech. 858, 71-90.

REYNOLDS, A. 1962 Forced oscillations in a rotating liquid (II). Z. Angew Math. Phys. 13, 561-572.

RieUtord, M., GEORGEOT, B. \& VALDETTARO, L. 2001 Inertial waves in a rotating spherical shell: attractors and asymptotic spectrum. J. Fluid Mech. 435, 103-144.

SARMA, L. V. K. V. \& KRISHNA, D. V. 1972 Oscillation of axisymmetric bodies in a stratified fluid. Zastosow. Matem. 13, 109-121.

SHMAKOVA, N., ERMANYUK, E. \& FLÓR, J.-B. 2017 Generation of higher harmonic internal waves by oscillating spheroids. Phys. Rev. Fluids 2, 114801.

SibgATUlLin, I. N. \& ERMANYUK, E. V. 2019 Internal and inertial wave attractors: a review. J. Appl. Mech. Tech. Phys. 60, 284-302.

SKAZKA, V. V. 1981 Asymptotic estimates for $t \rightarrow \infty$ of mixed problems for an equation of mathematical physics. Sib. Math. J. 22, 95-106.

St. LAURENT, L. \& GARRETT, C. 2002 The role of internal tides in mixing the deep ocean. J. Phys. Oceanogr. 32, 2882-2899.

StURova, I. V. 2001 Oscillations of a circular cylinder in a linearly stratified fluid. Fluid Dyn. 36, 478-488.

STUROVA, I. V. 2006 Oscillations of a cylinder piercing a linearly stratified fluid layer. Fluid Dyn. 41, 619-628.

STURovA, I. V. 2011 Hydrodynamic loads acting on an oscillating cylinder submerged in a stratified fluid with ice cover. J. Appl. Mech. Tech. Phys. 52, 415-426.

Sutherland, B. R. 2010 Internal Gravity Waves. Cambridge University Press.

Sutherland, B. R., DAlziel, S. B., Hughes, G. O. \& Linden, P. F. 1999 Visualization and measurement of internal waves by 'synthetic schlieren'. Part 1. Vertically oscillating cylinder. J. Fluid Mech. 390, 93-126.

Sutherland, B. R., FLYNN, M. R. \& ONU, K. 2003 Schlieren visualisation and measurement of axisymmetric disturbances. Nonlinear Process. Geophys. 10, 303-309.

Sutherland, B. R., Hughes, G. O., DAlZiel, S. B. \& LindEN, P. F. 2000 Internal waves revisited. Dyn. Atmos. Oceans 31, 209-232.

SUTHERLAND, B. R. \& LINDEN, P. F. 2002 Internal wave excitation by a vertically oscillating elliptical cylinder. Phys. Fluids 14, 721-731.

TABAEI, A. \& AKYLAS, T. R. 2003 Nonlinear internal gravity wave beams. J. Fluid Mech. 482, 141-161.

TABAEI, A., AKYLAS, T. R. \& LAMB, K. G. 2005 Nonlinear effects in reflecting and colliding internal wave beams. J. Fluid Mech. 526, 217-243.

THOMAS, N. H. \& STEVENSON, T. N. 1972 A similarity solution for viscous internal waves. J. Fluid Mech. 54, 495-506. 
TILGNER, A. 2000 Oscillatory shear layers in source driven flows in an unbounded rotating fluid. Phys. Fluids 12, 1101-1111.

VASIL'EV, A. YU. \& CHASHECHKIN, YU. D. 2003 The generation of beams of three-dimensional periodic internal waves in an exponentially stratified fluid. J. Appl. Math. Mech. 67, 397-405.

VASIL'EV, A. YU. \& CHASHECHKIN, YU. D. $2006 a$ Generation of beams of three-dimensional periodic internal waves by sources of various types. J. Appl. Mech. Tech. Phys. 47, 314-323.

VASIL'EV, A. YU. \& CHASHECHKIN, YU. D. $2006 b$ The generation of three-dimensional internal waves and attendant boundary layers in a viscous continuously stratified fluid. Construction of an analytical solution. Fluid Dyn. 41, 949-956.

VASIL'EV, A. YU. \& CHASHECHKIN, YU. D. 2012 Three-dimensional periodic flows of an inhomogeneous fluid in the case of oscillations of part of an inclined plane. J. Appl. Math. Mech. 76, 302-309.

Vic, C., Naveira Garabato, A. C., Green, J. A. M., Waterhouse, A. F., Zhao, Z., Melet, A., DE LaVergne, C., Buijsman, M. C. \& StePhenson, G. R. 2019 Deep-ocean mixing driven by small-scale internal tides. Nature Comm. 10, 2099.

VoIsIN, B. 1991 Internal wave generation in uniformly stratified fluids. Part 1. Green's function and point sources. J. Fluid Mech. 231, 439-480.

VoIsIN, B. 2003 Limit states of internal wave beams. J. Fluid Mech. 496, 243-293.

VoIsın, B. 2009 Added mass in density-stratified fluids. In 19ème Congrès Français de Mécanique (ed. C. Rey, P. Bontoux \& A. Chrisochoos); http://hdl.handle.net/2042/37312.

VOISIN, B., ERMANYUK, E. V. \& FLÓR, J.-B. 2011 Internal wave generation by oscillation of a sphere, with application to internal tides. J. Fluid Mech. 666, 308-357.

WALTON, I. C. 1975 On waves in a thin rotating spherical shell of slightly viscous fluid. Mathematika 22, 46-59.

WeStERWEEL, J. 1997 Fundamentals of digital particle image velocimetry. Meas. Sci. Technol. 8, 1379-1392.

WINTERS, K. B. \& ARMI, L. 2013 The response of a continuously stratified fluid to an oscillating flow past an obstacle. J. Fluid Mech. 727, 83-118.

ZHANG, H. P., KING, B. \& SWINNEY, H. L. 2007 Experimental study of internal gravity waves generated by supercritical topography. Phys. Fluids 19, 096602. 